\title{
Equilibrium density matrices for the 2D black hole sigma models from an integrable spin chain
}

\author{
Vladimir V. Bazhanov, ${ }^{a}$ Gleb A. Kotousov ${ }^{b}$ and Sergei L. Lukyanov ${ }^{c, d}$ \\ ${ }^{a}$ Department of Theoretical Physics, Research School of Physics and Engineering, \\ Australian National University, \\ 60 Mills rd., Canberra, ACT 2601, Australia \\ ${ }^{b}$ DESY, Theory Group, \\ Notkestrasse 85, Hamburg 22607, Germany \\ ${ }^{c}$ NHETC, Department of Physics and Astronomy, Rutgers University, \\ 136 Frelinghuysen rd., Piscataway, NJ 08855-0849, U.S.A. \\ ${ }^{d}$ Department of Mathematics, Kharkevich Institute for Information Transmission Problems, \\ Bol'shoi Karetnyi per. 19, Moscow 127994, Russia \\ E-mail: vladimir.bazhanov@anu.edu.au, gleb.kotousov@desy.de, \\ sergei@physics.rutgers.edu
}

ABSTRACT: This work concerns the quantum Lorentzian and Euclidean black hole nonlinear sigma models. For the Euclidean black hole sigma model an equilibrium density matrix is proposed, which reproduces the modular invariant partition function from the 2001 paper of Maldacena, Ooguri and Son. For the Lorentzian black hole sigma model, using its formulation as a gauged $\operatorname{SL}(2, \mathbb{R})$ WZW model, we describe the linear and Hermitian structure of its space of states and also propose an expression for the equilibrium density matrix. Our analysis is guided by the results of the study of a certain critical, integrable spin chain. In the scaling limit, the latter exhibits the key features of the Lorentzian black hole sigma model including the same global symmetries, the same algebra of extended conformal symmetry and a continuous spectrum of conformal dimensions.

Keywords: Black Holes in String Theory, Field Theories in Lower Dimensions, Sigma Models

ARXIV EPRINT: 2010.10603 


\section{Contents}

1 Introduction 1

2 Classical gauged $\mathrm{SL}(2, \mathbb{R})$ WZW model $\quad 4$

2.1 The classical action 4

2.2 Generalized boundary conditions 5

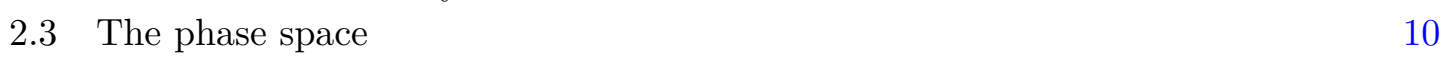

3 Quantum gauged SL(2, R) WZW model 12

$\begin{array}{lll}3.1 & \text { BRST quantization } & 12\end{array}$

$\begin{array}{lll}3.2 W_{\infty} \text {-algebra } & 15\end{array}$

$\begin{array}{lll}3.3 & \text { Space of states } & 17\end{array}$

4 Lorentzian black hole NLSM $\quad 19$

$\begin{array}{ll}4.1 \text { Space of states } & 19\end{array}$

$\begin{array}{ll}4.2 & \text { Minisuperspace approximation } \\ \end{array}$

5 Low energy states of the $\mathcal{Z}_{2}$ invariant spin chain in the scaling limit. $\begin{array}{ll}\text { Continuous spectrum } & 26\end{array}$

5.1 Global symmetries and Hermitian structure 26

$\begin{array}{ll}5.2 \text { Density of states } & 29\end{array}$

6 Density matrix for the Lorentzian black hole NLSM 32

7 Low energy states of the $\mathcal{Z}_{2}$ invariant spin chain in the scaling limit.

$\begin{array}{ll}\text { Discrete spectrum } & 34\end{array}$

7.1 Decomposition into the irreps of the algebra of extended conformal symmetry 34

$\begin{array}{lll}7.2 & \text { Hermitian structure } & 37\end{array}$

8 Density matrix for the Euclidean black hole NLSM 39

9 Conclusion $\quad 44$

\section{Introduction}

It was observed by Witten in ref. [1] that the two dimensional space equipped with the metric

$$
\left(\mathrm{d} \sigma_{\mathrm{LBH}}\right)^{2}=\frac{\mathrm{d} U \mathrm{~d} V}{1-U V}
$$

exhibits the characteristic features of a black hole geometry. As depicted in the space-time diagram in figure 1 , it possesses a horizon at $U V=0$ as well as a curvature singularity at 


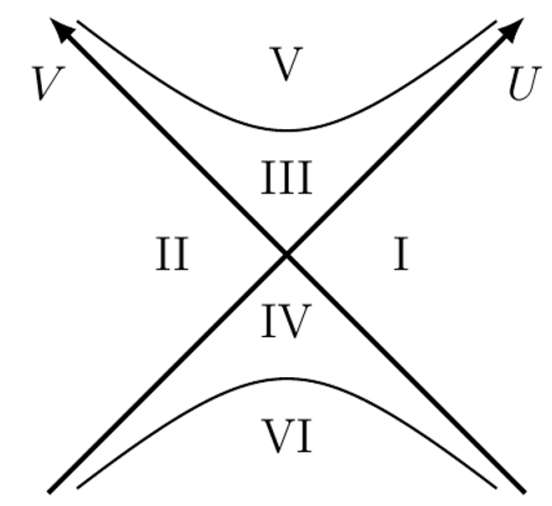

Figure 1. Space-time diagram for the Lorentzian black hole (1.1). The cross defined by the equation $U V=0$ is a horizon, while the metric possesses a physical singularity on the hyperbola $U V=1$.

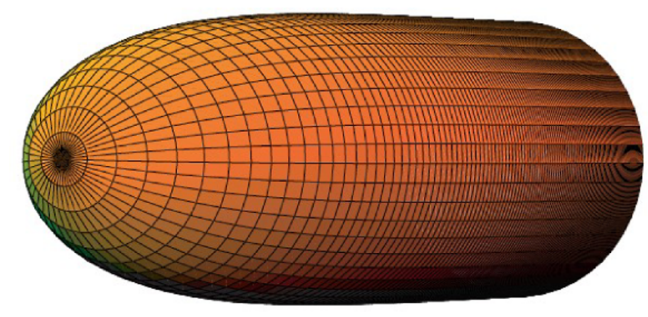

Figure 2. Visualization of the $2 D$ manifold with metric (1.2).

$U V=1$ just as the four dimensional Schwarzschild black hole in terms of Kruskal coordinates. There is no globally defined time coordinate for the metric. Rather, there is a Killing vector that is time-like only in regions I and II of figure 1 and space-like in regions III and IV.

Similar to the $3+1$ dimensional black hole, the Euclidean version of (1.1) is of prime interest. The latter can be introduced in the spirit of the Hartle-Hawking analytic continuation [2]. It is performed by a "Wick rotation" of the Killing coordinate $T=\frac{1}{2} \log (-U / V)$ from region I to pure imaginary values. This makes $U$ and $V$ satisfy the reality condition $V=-U^{*}$. Then, ignoring the overall negative sign, the Lorentzian metric becomes one of Euclidean signature:

$$
\left(\mathrm{d} \sigma_{\mathrm{EBH}}\right)^{2}=\frac{\mathrm{d} U \mathrm{~d} U^{*}}{1+U U^{*}} .
$$

The manifold equipped with $\left(\mathrm{d} \sigma_{\mathrm{EBH}}\right)^{2}$ may be embedded into three dimensional Euclidean space and visualized as a half-infinite cigar as shown in figure 2. The tip is located at $U=0$ while in the domain $|U| \gg 1$, where the metric becomes flat, the target manifold resembles a half-infinite cylinder. 
For each of the metrics $\left(\mathrm{d} \sigma_{\mathrm{LBH}}\right)^{2}$ and $\left(\mathrm{d} \sigma_{\mathrm{EBH}}\right)^{2}$ one can associate a Non-Linear Sigma Model (NLSM) whose classical dynamics is governed by the action ${ }^{1}$

$$
\begin{aligned}
& S_{\mathrm{LBH}}=\frac{1}{2 \hbar} \int \mathrm{d}^{2} x \frac{\partial_{\mu} U \partial^{\mu} V}{1-U V} \\
& S_{\mathrm{EBH}}=\frac{1}{2 \hbar} \int \mathrm{d}^{2} x \frac{\partial_{\mu} U \partial^{\mu} U^{*}}{1+U U^{*}} .
\end{aligned}
$$

It is a challenging problem, in general, to assign a meaning to a quantum NLSM whose target space metric is of Lorentzian signature. In particular the status of the quantum Lorentzian black hole NLSM is rather tentative. Contrary to this the quantum Euclidean black hole NLSM is a well studied CFT [1, 3-12].

In refs. $[9,10]$ an explicit formula was presented for the partition function $Z_{\mathrm{EBH}}$. The latter is defined as a Euclidean path integral involving the Euclidean action with the worldsheet compactified on a torus. The partition function contains a divergence which comes from the contribution of the asymptotically flat domain of the target manifold:

$$
Z_{\mathrm{EBH}}=Z_{\epsilon}^{(\text {sing) }}+Z_{\mathrm{EBH}}^{(\mathrm{reg})} .
$$

The singular part is somewhat universal. It coincides with the partition function of two free bosons, one of them being compactified, $\arg (U) \sim \arg (U)+2 \pi$, while the other, $\log |U|$, taking values in a segment of length $\propto \log (1 / \epsilon)$. Thus, as the regularization parameter $\epsilon \rightarrow 0$,

$$
Z_{\epsilon}^{\text {(sing) }} \propto \log (1 / \epsilon) \rightarrow+\infty .
$$

On the other hand, the finite part $Z_{\mathrm{EBH}}^{(\mathrm{reg})}$ in (1.4) depends on the infra red regularization of the target manifold, which can be thought of as being the result of interactions with an external thermostat. In the works [8-10] the Euclidean black hole NLSM occurs in the context of bosonic string theory on $\mathrm{AdS}_{3}$. This provides a particular regularization. Its major advantage, which makes it especially attractive from the stringy point of view, is that $Z_{\mathrm{EBH}}$ is invariant w.r.t. modular transformations of the world-sheet torus. Then an immediate question arises concerning the full equilibrium density matrix $\hat{\rho}_{\mathrm{EBH}}$ corresponding to such a regularization. The latter can not be recovered from just the knowledge of the partition function, which is given by the trace of $\hat{\rho}_{\mathrm{EBH}} \cdot{ }^{2} \mathrm{~A}$ conjecture for $\hat{\rho}_{\mathrm{EBH}}$ was put forward in the original work [9]. However numerical analysis shows that the proposed expression fails to reproduce the modular invariant partition function.

In ref. [27] Ikhlef, Jacobsen and Saleur made the interesting proposal that the Euclidean black hole NLSM is the CFT governing the scaling limit of a certain integrable spin chain. This opened a potential way of obtaining the equilibrium density matrix $\hat{\rho}_{\mathrm{EBH}}$. In the recent

\footnotetext{
${ }^{1}$ In this paper we use the following conventions. We assume Minkowski signature for the world-sheet $\left(x^{0}, x^{1}\right) \equiv(t, x)$ so that $\partial_{0}=\partial^{0} \equiv \frac{\partial}{\partial x^{0}}, \partial_{1}=-\partial^{1} \equiv \frac{\partial}{\partial x^{1}}$ with the space co-ordinate belonging to the segment $x \in[0,2 \pi]$. The integration measure is taken to be $\mathrm{d}^{2} x=\mathrm{d} x^{0} \mathrm{~d} x^{1}$. The Plank constant is a positive dimensionless parameter. We'll also use the Levi-Civita symbol $\epsilon_{\mu \nu}=-\epsilon^{\mu \nu}$, below, which is defined as $\epsilon_{01}=-\epsilon_{10}=1$.

${ }^{2}$ We use the non-standard convention that the trace of the (equilibrium) density matrix rather than being one, coincides with the partition function.
} 
work [32] an extensive study of the spin chain was performed and the original conjecture from [27] was re-examined. Here, based on the results of that work, we address the following questions:

(i) What is the correct expression for $\hat{\rho}_{\mathrm{EBH}}$ ?

(ii) What is the space of states of the Lorentzian black hole NLSM and how to assign to it a meaningful equilibrium density matrix $\hat{\rho}_{\mathrm{LBH}}$ ? In turn, how would the latter be related to its Euclidean counterpart?

\section{Classical gauged $\mathrm{SL}(2, \mathbb{R})$ WZW model}

\subsection{The classical action}

The Lorentzian black hole NLSM can be obtained by gauging a non-compact one dimensional subgroup of the classical $\mathrm{SL}(2, \mathbb{R})$ WZW model. Following the work [1] consider the classical action

$$
\begin{aligned}
S=\frac{1}{\hbar} \int \mathrm{d}^{2} x \frac{1}{2}[ & \partial_{\mu} U \partial^{\mu} V+\partial_{\mu} X \partial^{\mu} Y+\log (X / Y) \epsilon^{\mu \nu} \partial_{\mu} U \partial_{\nu} V \\
& \left.+a_{\mu}\left(Y \partial^{\mu} X-X \partial^{\mu} Y\right)+\epsilon^{\mu \nu} a_{\mu}\left(U \partial_{\nu} V-V \partial_{\nu} U\right)-a_{\mu} a^{\mu} X Y\right] .
\end{aligned}
$$

Here the integrand in the first line is just the classical Lagrangian density of the usual WZW model [13], $\mathcal{L}_{\mathrm{WZW}}[\mathbf{g}]$, expressed via the matrix entries of the fundamental WZW field

$$
\mathbf{g}=\left(\begin{array}{cc}
X & U \\
-V & Y
\end{array}\right) \in \mathrm{SL}(2, \mathbb{R})
$$

Note that the term involving $\log (X / Y)$ comes from the Wess-Zumino term and, up to a total derivative, can be rewritten in various ways by employing the constraint

$$
X Y+U V=1 .
$$

The second line in (2.1) contains the gauge potential $a_{\mu}$. The action is invariant w.r.t. the infinitesimal gauge transformation of the form

$$
\delta X=\delta \omega X, \quad \delta Y=-\delta \omega Y, \quad \delta U=\delta V=0 ; \quad \delta a_{\mu}=\partial_{\mu}(\delta \omega)
$$

This can be seen by rewriting the Lagrangian density corresponding to the action (2.1) as

$$
\mathcal{L}=\frac{1}{2}\left[\frac{\partial_{\mu} U \partial^{\mu} V}{1-U V}-(1-U V) f_{\mu} f^{\mu}+\epsilon^{\mu \nu} \partial_{\mu} C_{\nu}\right]
$$

with

$$
f_{\mu}=a_{\mu}-\frac{1}{2} \partial_{\mu} \log (X / Y)-\epsilon_{\mu \nu} J^{\nu}, \quad C_{\mu}=\frac{1}{2} \log (X / Y)\left(U \partial_{\mu} V-V \partial_{\mu} U\right)
$$

and

$$
J_{\mu}=\frac{1}{2} \frac{U \partial_{\mu} V-V \partial_{\mu} U}{1-U V} .
$$


The extremum condition $\frac{\delta}{\delta a_{\mu}} S=0$ leads to the equation

$$
a_{\mu}=\frac{1}{2} \partial_{\mu} \log (X / Y)+\epsilon_{\mu \nu} J^{\nu}
$$

The field strength corresponding to this vector potential is given by

$$
\partial_{\mu} a_{\nu}-\partial_{\nu} a_{\mu}=\partial_{\sigma} J^{\sigma} \epsilon_{\mu \nu} .
$$

It vanishes for any solution of the classical equations of motion, which includes the continuity equation $\partial_{\mu} J^{\mu}=0$.

In the orthodox formulation of the gauged $\operatorname{SL}(2, \mathbb{R})$ WZW model, the matrix valued field $\mathbf{g}$ is assumed to be periodic:

$$
\mathbf{g}(t, x+2 \pi)=\mathbf{g}(t, x) .
$$

If we take $U$ and $V$ from the domain

$$
0 \leq U V<1
$$

it is natural to fix the gauge by setting [1]

$$
X=Y
$$

which, in view of eq. (2.8), results in $a_{\mu}=\epsilon_{\mu \nu} J^{\nu}$. Then, after eliminating the auxiliary field $a_{\mu}$, the action $S$ (2.1) becomes that of the Lorentzian black hole NLSM (1.3a). Note that, as was also pointed out in [1], if we take the $\operatorname{SL}(2, \mathbb{R})$ picture literally the full target space of the Lorentzian black hole NLSM would contain two copies of the regions III and IV in figure 1 corresponding to the cases $X, Y>0$ and $X, Y<0$.

\subsection{Generalized boundary conditions}

In what follows we'll consider the gauged $\mathrm{SL}(2, \mathbb{R})$ WZW model, but with more general boundary conditions than (2.10). It is instructive to discuss the latter using another formulation of the classical field theory $[6,14]$. In this description the Lagrange density is just the sum of that of the WZW model and the massless Gaussian theory:

$$
\tilde{\mathcal{L}}=\mathcal{L}_{\mathrm{WZw}}[\mathbf{G}]+2 \partial \eta \bar{\partial} \eta
$$

The interaction is introduced through the constraints

$$
\Upsilon \equiv \frac{1}{2} \operatorname{Tr}\left[\sigma^{z} \partial \mathbf{G ~ G}^{-1}\right]-\partial \eta=0, \quad \bar{\Upsilon} \equiv \frac{1}{2} \operatorname{Tr}\left[\sigma^{z} \mathbf{G}^{-1} \bar{\partial} \mathbf{G}\right]+\bar{\partial} \eta=0,
$$

where we use $\partial=\frac{1}{2}\left(\partial_{0}+\partial_{1}\right)$ and $\bar{\partial}=\frac{1}{2}\left(\partial_{0}-\partial_{1}\right)$. If the infinitesimal gauge transformation of the WZW field and the massless Gaussian field is defined as

$$
\delta \mathbf{G}=\frac{1}{2}\left(\sigma^{z} \mathbf{G}+\mathbf{G} \sigma^{z}\right) \delta \omega, \quad \partial_{\mu} \delta \eta=\epsilon_{\mu \nu} \partial^{\nu}(\delta \omega),
$$


then $\delta \tilde{\mathcal{L}}$ turns out to be a total derivative provided the constraints (2.14) are imposed. The classical field theory, thus defined, is equivalent to the gauged WZW model governed by the action (2.1). In particular for any field configuration satisfying the equations of motion for (2.13), (2.14),

$$
\mathbf{g}=\mathrm{e}^{\frac{1}{2} \omega \sigma^{z}} \mathbf{G} \mathrm{e}^{\frac{1}{2} \omega \sigma^{z}}, \quad a_{\mu}=-\epsilon_{\mu \nu} \partial^{\nu} \eta+\partial_{\mu} \omega
$$

would be a solution of the Euler-Lagrange equations associated with the action (2.1). Here $\omega$ is an arbitrary periodic function $\omega(t, x+2 \pi)=\omega(t, x)$, which appears as a manifestation of the gauge invariance of the model.

To specify the boundary conditions, let us first recall some basic facts concerning the phase space of the WZW model (see, e.g., $[13,15,16]$ ). The latter is conveniently described in terms of the left and right WZW currents, ${ }^{3}$

$$
\partial \mathbf{G G}^{-1}=L^{A} \mathbf{t}_{A}, \quad \mathbf{G}^{-1} \bar{\partial} \mathbf{G}=\bar{R}^{A} \mathbf{t}_{A},
$$

which satisfy the closed set of equal-time Poisson Bracket relations:

$$
\begin{aligned}
\left\{L^{A}(t, x), L^{B}(t, y)\right\} & =-\frac{1}{2} \kappa^{A B} \delta^{\prime}(x-y)-\frac{1}{2} f_{C}^{A B} L^{C}(t, x) \delta(x-y) \\
\left\{\bar{R}^{A}(t, x), \bar{R}^{B}(t, y)\right\} & =+\frac{1}{2} \kappa^{A B} \delta^{\prime}(x-y)+\frac{1}{2} f^{A B}{ }_{C} \bar{R}^{C}(t, x) \delta(x-y) \\
\left\{L^{A}(t, x), R^{B}(t, y)\right\} & =0 .
\end{aligned}
$$

Assuming that the currents are periodic fields,

$$
L^{A}(t, x+2 \pi)=L^{A}(t, x), \quad \bar{R}^{A}(t, x+2 \pi)=\bar{R}^{A}(t, x),
$$

the center of the Poisson algebra is generated by two elements

$$
\mathfrak{C}=\operatorname{Tr}\left[\overleftarrow{\mathcal{P}} \exp \left(+\int_{x_{0}}^{x_{0}+2 \pi} \mathrm{d} x L^{A} \mathbf{t}_{A}\right)\right], \quad \overline{\mathfrak{C}}=\operatorname{Tr}\left[\overrightarrow{\mathcal{P}} \exp \left(-\int_{-x_{0}}^{-x_{0}-2 \pi} \mathrm{d} x \bar{R}^{A} \mathbf{t}_{A}\right)\right]
$$

We will focus on the field configurations such that the values of the central elements are restricted by the inequalities

$$
-2<\mathfrak{C}, \overline{\mathfrak{C}}<2
$$

and use the parameterization

$$
\mathfrak{C}=2 \cos (2 \pi P), \quad \overline{\mathfrak{C}}=2 \cos (2 \pi \bar{P})
$$

\footnotetext{
${ }^{3}$ Here and below we use the notation $\mathbf{t}_{A}$ for the $2 \times 2$ real traceless matrices,
}

$$
\mathbf{t}_{3}=\left(\begin{array}{cc}
1 & 0 \\
0 & -1
\end{array}\right), \quad \mathbf{t}_{+}=\left(\begin{array}{ll}
0 & 1 \\
0 & 0
\end{array}\right), \quad \mathbf{t}_{-}=\left(\begin{array}{ll}
0 & 0 \\
1 & 0
\end{array}\right): \quad\left[\mathbf{t}_{A}, \mathbf{t}_{B}\right]=f_{A B}^{C} \mathbf{t}_{C}
$$

Indices are raised and lowered via the Killing form defined as

$$
\kappa_{A B}=\frac{1}{2} \operatorname{Tr}\left[\mathbf{t}_{A} \mathbf{t}_{B}\right], \quad \kappa_{A C} \kappa^{C B}=\delta_{A}^{B} .
$$


with real $P$ and $\bar{P}$. In this case the path ordered exponentials inside the traces in $(2.20)$ may be expressed as

$$
\begin{aligned}
\overleftarrow{\mathcal{P}} \exp \left(+\int_{x_{0}}^{x_{0}+2 \pi} \mathrm{d} x L^{A} \mathbf{t}_{A}\right) & =\boldsymbol{M} \mathrm{e}^{+2 \pi \mathrm{i} P \sigma^{y}} \boldsymbol{M}^{-1} \\
\overrightarrow{\mathcal{P}} \exp \left(-\int_{-x_{0}}^{-x_{0}-2 \pi} \mathrm{d} x \bar{R}^{A} \mathbf{t}_{A}\right) & =\overline{\boldsymbol{M}} \mathrm{e}^{-2 \pi \mathrm{i} \bar{P} \sigma^{y}} \overline{\boldsymbol{M}}^{-1}
\end{aligned}
$$

where the $2 \times 2$ real non-degenerate matrices $\boldsymbol{M}$ and $\overline{\boldsymbol{M}}$ depend on the initial integration point $x_{0}$. If we require them to be $\operatorname{SL}(2, \mathbb{R})$ matrices, then $\mathrm{e}^{+2 \pi \mathrm{i} P \sigma^{y}}$ and $\mathrm{e}^{-2 \pi \mathrm{i} \bar{P} \sigma^{y}}$ are uniquely defined. At the same time there is an ambiguity in $\boldsymbol{M}$ and $\overline{\boldsymbol{M}}$ of the form $\boldsymbol{M} \mapsto \pm \boldsymbol{M} \mathrm{e}^{\mathrm{i} \phi \sigma^{y}}$ and $\overline{\boldsymbol{M}} \mapsto \pm \overline{\boldsymbol{M}} \mathrm{e}^{\mathrm{i} \bar{\phi} \sigma^{y}}$ with arbitrary real $\phi$ and $\bar{\phi}$. This can be fixed using the Iwasawa decomposition for $\operatorname{SL}(2, \mathbb{R})$ matrices, which allows one to specify that

$$
\boldsymbol{M}=\left(\begin{array}{cc}
d & 0 \\
0 & d^{-1}
\end{array}\right)\left(\begin{array}{ll}
1 & b \\
0 & 1
\end{array}\right), \quad \overline{\boldsymbol{M}}=\left(\begin{array}{cc}
\bar{d} & 0 \\
0 & \bar{d}^{-1}
\end{array}\right)\left(\begin{array}{ll}
1 & \bar{b} \\
0 & 1
\end{array}\right) \quad \text { with } \quad d, \bar{d}>0
$$

The values of the currents at $t=0$ are not enough to fully define the time dependence of the matrix valued field $\mathbf{G}(t, x)$. Indeed the equations of motion in the WZW model are given by

$$
\bar{\partial} L^{A}=0, \quad \partial \bar{R}^{A}=0 .
$$

This implies that

$$
\mathbf{G}(t, x)=\mathbf{\Omega}(t+x) \mathbf{G}\left(0, x_{0}\right) \overline{\boldsymbol{\Omega}}(t-x),
$$

where

$$
\begin{aligned}
& \boldsymbol{\Omega}(u)=\overleftarrow{\mathcal{P}} \exp \left(+\int_{x_{0}}^{u} \mathrm{~d} x L^{A} \mathbf{t}_{A}\right) \\
& \overline{\boldsymbol{\Omega}}(\bar{u})=\overrightarrow{\mathcal{P}} \exp \left(-\int_{-x_{0}}^{\bar{u}} \mathrm{~d} x \bar{R}^{A} \mathbf{t}_{A}\right)
\end{aligned}
$$

while $\mathbf{G}\left(0, x_{0}\right)$ is an arbitrary $\mathrm{SL}(2, \mathbb{R})$ matrix. Its entries, together with the initial values of the currents, constitute the full set of the initial data. We consider the field configurations at $t=0$ to be such that

$$
\mathbf{G}\left(0, x_{0}\right)=\boldsymbol{M} \mathrm{e}^{\mathrm{i} \alpha \sigma^{y}} \overline{\boldsymbol{M}}^{-1},
$$

where $\boldsymbol{M}, \overline{\boldsymbol{M}}$ are the same as in (2.23), (2.24) and $\alpha$ is some real number. This is motivated through the following arguments. Assuming $L^{A}$ are given, the path ordered exponent $\boldsymbol{\Omega}(u)(2.27)$ solves the linear differential equation

$$
\partial \Psi=L^{A} \mathbf{t}_{A} \boldsymbol{\Psi}
$$

However $\boldsymbol{\Omega}(u)$, apart from the WZW currents, also depends on an arbitrarily chosen initial integration point $x_{0}$ at which it becomes the identity matrix. At the same time $\boldsymbol{\Psi}_{P}=$ $\boldsymbol{\Omega}(u) \boldsymbol{M}$ is the Floquet solution of the matrix ODE (2.29), which is fixed unambiguously provided $\boldsymbol{M}$ is taken to be of the form (2.24). A change in the initial integration point $x_{0}$ to $x_{0}^{\prime}$ would result in the transformation $\Psi_{P} \mapsto \Psi_{P} \mathrm{e}^{\mathrm{i} \alpha_{0} \sigma^{y}}$, where $\alpha_{0}=\alpha_{0}\left(x_{0}, x_{0}^{\prime}\right)$. 
The solutions of the ODE with periodic coefficients possess the band structure. Thus the parameter $P$ labeling the Floquet solutions $\Psi_{P}$ can be defined such that $P \in \mathbb{R}$ and $2 P \notin \mathbb{Z}$, where the band number coincides with the greatest integer less than $P+\frac{1}{2}$. The above carries over to the Floquet solution $\overline{\mathbf{\Psi}}_{\bar{P}}=\overline{\boldsymbol{M}}^{-1} \overline{\boldsymbol{\Omega}}(\bar{u})$ of the barred counterpart of the ODE (2.29). This way the construction of the WZW field $\mathbf{G}(t, x)$ given by eqs. (2.26) and (2.28) involves the Floquet solutions as well as an additional variable $\alpha \sim \alpha+2 \pi$. Thus the algebra of functions on the phase space of the WZW model, generated by the currents $L^{A}$ and $\bar{R}^{A}$ subject to the periodic boundary conditions (2.19), should be extended by the inclusion of the compact generalized coordinate $\alpha$. The latter can be viewed as a dynamical variable canonically conjugated to the sum $2 \pi(P+\bar{P})$. As for the difference, we assume that $\mathrm{e}^{2 \pi \mathrm{i}(P-\bar{P})}=\mathrm{e}^{2 \pi \mathrm{ik}}$, with $\frac{1}{2}<\mathrm{k} \leq \frac{1}{2}$ being a fixed parameter. Equivalently,

$$
P-\bar{P}=\mathrm{k}+\mathrm{w} \quad(\mathrm{w} \in \mathbb{Z})
$$

so that the integer w labels different disjoint components of the phase space.

The boundary values of the WZW field at $t=0$, defined by the formulae (2.26) and (2.28), satisfy the relations

$$
\mathbf{G}\left(0, x_{0}+2 \pi\right)=\boldsymbol{M} \mathrm{e}^{2 \pi \mathrm{ik} \sigma^{y}} \boldsymbol{M}^{-1} \mathbf{G}\left(0, x_{0}\right)=\mathbf{G}\left(0, x_{0}\right) \overline{\boldsymbol{M}} \mathrm{e}^{2 \pi \mathrm{ik} \sigma^{y}} \overline{\boldsymbol{M}}^{-1} .
$$

This implies

$$
\operatorname{Tr}\left[\mathbf{G}(t, x+2 \pi)(\mathbf{G}(t, x))^{-1}\right]=2 \cos (2 \pi \mathrm{k}),
$$

which should be imposed along with the periodicity condition for the currents (2.19). In fact there is an extra condition which needs to be taken into account. Substituting the matrix $\boldsymbol{M}$ (2.24) into eq. (2.31) one finds

$$
\operatorname{Tr}\left[\left(-\mathrm{i} \sigma^{y}\right) \mathbf{G}\left(0, x_{0}+2 \pi\right)\left(\mathbf{G}\left(0, x_{0}\right)\right)^{-1}\right]=\sin (2 \pi \mathrm{k})\left(d^{2}+d^{-2}+d^{2} b^{2}\right) .
$$

This results in the inequality

$$
\operatorname{Tr}\left[\left(-\mathrm{i} \sigma^{y}\right) \mathbf{G}(t, x+2 \pi)(\mathbf{G}(t, x))^{-1}\right] / \sin (2 \pi \mathrm{k})>0 .
$$

The constraints (2.14) will only make sense when both derivatives $\partial \eta$ and $\bar{\partial} \eta$ are periodic:

$$
\partial \eta(t, x+2 \pi)=\partial \eta(t, x), \quad \bar{\partial} \eta(t, x+2 \pi)=\bar{\partial} \eta(t, x) .
$$

In view of the relation $(2.16)$, the gauge field $a_{\mu}(x, t)$ in the original formulation of the gauged WZW model is also periodic,

$$
a_{\mu}(t, x+2 \pi)=a_{\mu}(t, x),
$$

as was implicitly assumed in our initial analysis of the model. The boundary condition (2.32) as well as the inequality (2.34) are invariant under the gauge transformation and therefore the field $\mathbf{g}$ satisfies the similar relations

$$
\begin{aligned}
\operatorname{Tr}\left[\mathbf{g}(t, x+2 \pi)(\mathbf{g}(t, x))^{-1}\right] & =2 \cos (2 \pi \mathrm{k}) \\
\operatorname{Tr}\left[\left(-\mathrm{i} \sigma^{y}\right) \mathbf{g}(t, x+2 \pi)(\mathbf{g}(t, x))^{-1}\right] / \sin (2 \pi \mathrm{k}) & >0 .
\end{aligned}
$$


Let us make the following important point. In the case of the gauged $\mathrm{SL}(2, \mathbb{R})$ WZW model with $\mathrm{k}=0$, the conditions (2.37) yield $\mathbf{g}(t, x+2 \pi)=\mathbf{g}(t, x)$, i.e., periodicity of all the matrix elements $X, Y, U, V$. In turn one can use the gauge fixing condition $X=Y$. However for $\mathrm{k} \neq 0$, since $X$ and $Y$ are no longer periodic fields, the same gauge fixing condition is not applicable. This makes the model with $\mathrm{k}=0$ (which is equivalent to the Lorentzian black hole NLSM) a very special one that is not obtainable literally through a naive $\mathrm{k} \rightarrow 0$ limit.

The Poisson structure of the massless Gaussian model, whose Lagrange density is given by the second term in the r.h.s. of (2.13), reads as

$$
\{\partial \eta(t, x), \partial \eta(t, y)\}=-\{\bar{\partial} \eta(t, x), \bar{\partial} \eta(t, y)\}=\frac{1}{2} \delta^{\prime}(x-y), \quad\{\partial \eta(t, x), \bar{\partial} \eta(t, y)\}=0 .
$$

With the boundary conditions (2.35) imposed, the center of this Poisson algebra is generated by

$$
P_{\eta}=\int_{0}^{2 \pi} \frac{\mathrm{d} x}{2 \pi} \partial \eta, \quad \bar{P}_{\eta}=\int_{0}^{2 \pi} \frac{\mathrm{d} x}{2 \pi} \bar{\partial} \eta .
$$

The general solution of the equation of motion $\partial \bar{\partial} \eta=0$ is

$$
\eta(t, x)=\frac{1}{2}(f(t+x)-\bar{f}(t-x))
$$

where, in view of the boundary conditions, the arbitrary functions $f$ and $\bar{f}$ are quasiperiodic:

$$
f(u+2 \pi)=f(u)+P_{\eta}, \quad \bar{f}(\bar{u}+2 \pi)=\bar{f}(\bar{u})+\bar{P}_{\eta} .
$$

The constraints (2.14) imposed on the WZW field G and the Gaussian field, combined with (2.40), yield the relations

$$
L^{3}=-\frac{1}{2} \partial f, \quad \bar{R}^{3}=-\frac{1}{2} \bar{\partial} \bar{f} .
$$

It is easy to see now that the matrix $\mathbf{G}$, satisfying the equations of motion, can be brought to the form

$$
\mathbf{G}(t, x)=\mathrm{e}^{-\frac{1}{2} f(t+x) \sigma^{z}} \mathfrak{G}(t, x) \mathrm{e}^{-\frac{1}{2} \bar{f}(t-x) \sigma^{z}},
$$

where $\mathfrak{G}$ is such that

$$
\partial \mathfrak{G} \mathfrak{G}^{-1}=\xi_{-} \mathbf{t}_{-}-\xi_{+} \mathbf{t}_{+}, \quad \quad \mathfrak{G}^{-1} \bar{\partial} \mathfrak{G}=\bar{\xi}_{-} \mathbf{t}_{-}-\bar{\xi}_{+} \mathbf{t}_{+}
$$

with

$$
\begin{array}{ll}
\xi_{-}=\mathrm{e}^{-f} L^{-}, & \xi_{+}=-\mathrm{e}^{+f} L^{+} \\
\bar{\xi}_{-}=\mathrm{e}^{+\bar{f}} \bar{R}^{-}, & \bar{\xi}_{+}=-\mathrm{e}^{-\bar{f}} \bar{R}^{+} .
\end{array}
$$

The latter are real chiral fields, $\bar{\partial} \xi_{ \pm}=\partial \bar{\xi}_{ \pm}=0$, subject to the quasiperiodic boundary conditions

$$
\xi_{ \pm}(u+2 \pi)=B^{ \pm 1} \xi_{ \pm}(u), \quad \bar{\xi}_{ \pm}(\bar{u}+2 \pi)=\bar{B}^{ \pm 1} \bar{\xi}_{ \pm}(\bar{u})
$$


where $B=\mathrm{e}^{2 \pi P_{\eta}}$ and $\bar{B}=\mathrm{e}^{2 \pi \bar{P}_{\eta}}$. We set $B=\bar{B}$ or, equivalently, $P_{\eta}=\bar{P}_{\eta}$ (assuming that $P_{\eta}$ and $\bar{P}_{\eta}$ are real). In this case, as it follows from eqs. (2.40) and (2.41), the field $\eta$ is periodic:

$$
\eta(t, x+2 \pi)=\eta(t, x) .
$$

Note that the on-shell gauge potential $a_{\mu}$, entering into the initial formulation of the $\mathrm{SL}(2, \mathbb{R})$ gauged WZW model, satisfies the condition

$$
B=\bar{B}=\exp \left(\oint \mathrm{d} x^{\mu} a_{\mu}\right) .
$$

\subsection{The phase space}

Consider now the classical fields defined through the relations

$$
\begin{array}{ll}
W_{2}^{(c l)}=\xi_{+} \xi_{-}, \quad W_{3}^{(c l)}=\frac{1}{2}\left(\xi_{-} \partial \xi_{+}-\xi_{+} \partial \xi_{-}\right), \\
\bar{W}_{2}^{(c l)}=\bar{\xi}_{+} \bar{\xi}_{-}, \quad \bar{W}_{3}^{(c l)}=\frac{1}{2}\left(\bar{\xi}_{-} \partial \bar{\xi}_{+}-\bar{\xi}_{+} \partial \bar{\xi}_{-}\right) .
\end{array}
$$

Using (2.45) they can be rewritten in terms of the WZW currents along with $\partial \eta$ and $\bar{\partial} \eta$ :

$$
\begin{array}{ll}
W_{2}^{(c l)}=(\partial \eta)^{2}-\left(\left(L^{3}\right)^{2}+L^{+} L^{-}\right), & W_{3}^{(c l)}=2 \partial \eta L^{+} L^{-}+\frac{1}{2}\left(L^{+} \partial L^{-}-L^{-} \partial L^{+}\right) \\
\bar{W}_{2}^{(c l)}=(\bar{\partial} \eta)^{2}-\left(\left(\bar{R}^{3}\right)^{2}+\bar{R}^{+} \bar{R}^{-}\right), & \bar{W}_{3}^{(c l)}=2 \bar{\partial} \eta \bar{R}^{+} \bar{R}^{-}+\frac{1}{2}\left(\bar{R}^{+} \partial \bar{R}^{-}-\bar{R}^{-} \bar{\partial} \bar{R}^{+}\right) .
\end{array}
$$

It is straightforward to check using the PB relations (2.18) and (2.38) that the $W$ fields Poisson commute (in a weak sense) with the constraints $\bar{\Upsilon}$ and $\Upsilon(2.14)$,

$$
\begin{aligned}
& \left.\left\{W_{j}^{(c l)}(t, x), \Upsilon(t, y)\right\}\right|_{\Upsilon=0}=\left\{W_{j}^{(c l)}(t, x), \bar{\Upsilon}(t, y)\right\}=0 \\
& \left.\left\{\bar{W}_{j}^{(c l)}(t, x), \bar{\Upsilon}(t, y)\right\}\right|_{\widehat{\Upsilon}=0}=\left\{\bar{W}_{j}^{(c l)}(t, x), \Upsilon(t, y)\right\}=0 .
\end{aligned}
$$

Since $W_{2}^{(c l)}$ and $\bar{W}_{2}^{(c l)}$ coincide with the nonvanishing components of the stress energy tensor, the Hamiltonian commutes with $\bar{\Upsilon}$ and $\Upsilon$. Also it is easy to see that

$$
\{\Upsilon(t, x), \Upsilon(t, y)\}=\{\bar{\Upsilon}(t, x), \bar{\Upsilon}(t, y)\}=\{\Upsilon(t, x), \bar{\Upsilon}(t, y)\}=0
$$

and, hence, the system of constraints (2.14) are of the first class. The $W$ fields are "classical observables" which are

(i) chiral

$$
W_{j}^{(c l)}=W_{j}^{(c l)}(t+x), \quad \bar{W}_{j}^{(c l)}=\bar{W}_{j}^{(c l)}(t-x)
$$

(ii) real

$$
\left(W_{j}^{(c l)}(u)\right)^{*}=W_{j}^{(c l)}\left(u^{*}\right), \quad\left(\bar{W}_{j}^{(c l)}(\bar{u})\right)^{*}=\bar{W}_{j}^{(c l)}\left(\bar{u}^{*}\right)
$$

(iii) periodic

$$
W_{j}^{(c l)}(u+2 \pi)=W_{j}^{(c l)}(u), \quad \bar{W}_{j}^{(c l)}(\bar{u}+2 \pi)=\bar{W}_{j}^{(c l)}(\bar{u}) .
$$


They generate a closed Poisson algebra in the following sense. Straightforward calculations lead to

$$
\begin{aligned}
& \left\{W_{2}^{(c l)}\left(u_{1}\right), W_{2}^{(c l)}\left(u_{2}\right)\right\}=-\left(W_{2}^{(c l)}\left(u_{1}\right)+W_{2}^{(c l)}\left(u_{2}\right)\right) \delta^{\prime}\left(u_{1}-u_{2}\right) \\
& \left\{W_{3}^{(c l)}\left(u_{1}\right), W_{2}^{(c l)}\left(u_{2}\right)\right\}=-3 W_{3}^{(c l)}\left(u_{1}\right) \delta^{\prime}\left(u_{1}-u_{2}\right)-\partial W_{3}^{(c l)}\left(u_{1}\right) \delta\left(u_{1}-u_{2}\right) \\
& \left\{W_{3}^{(c l)}\left(u_{1}\right), W_{3}^{(c l)}\left(u_{2}\right)\right\}=-\frac{1}{4}\left(W_{2}^{(c l)}\left(u_{1}\right)+W_{2}^{(c l)}\left(u_{2}\right)\right) \delta^{\prime \prime \prime}\left(u_{1}-u_{2}\right)-\delta^{\prime}\left(u_{1}-u_{2}\right) \\
& \times\left(W_{4}^{(c l)}\left(u_{1}\right)+W_{4}^{(c l)}\left(u_{2}\right)+2 W_{2}^{(c l)}\left(u_{1}\right) W_{2}^{(c l)}\left(u_{2}\right)-\frac{3}{20}\left(\partial^{2} W_{2}^{(c l)}\left(u_{1}\right)+\partial^{2} W_{2}^{(c l)}\left(u_{2}\right)\right)\right) .
\end{aligned}
$$

Here $W_{4}^{(c l)}$, which appears in the last line, is expressed in a form similar to (2.49):

$$
W_{4}^{(c l)}=\frac{2}{5}\left(\xi_{+} \partial^{2} \xi_{-}+\xi_{-} \partial^{2} \xi_{+}\right)-\frac{6}{5} \partial \xi_{+} \partial \xi_{-} .
$$

Analogous relations hold true for the "barred" fields. A recursive computation of the Poisson brackets of the $W$ fields amongst themselves yields two infinite sets $\left\{W_{j}^{(c l)}\right\}_{j=2}^{\infty}$ and $\left\{\bar{W}_{j}^{(c l)}\right\}_{j=2}^{\infty}$. We'll refer below to the fields from these sets as the (classical) $W$ currents. All of them satisfy the conditions (i)-(iii) as well as (2.51). The integer $j=2,3, \ldots$ coincides with the Lorentz spin of $W_{j}^{(c l)}$ while the Lorentz spin of $\bar{W}_{j}^{(c l)}$ is given by $(-j)$. The $W$ currents form a quadratic Poisson algebra [17]. Since all the Poisson bracket between the $W$ currents of different chirality vanish, we'll refer to it as the classical $\bar{W}_{\infty} \otimes W_{\infty}$-algebra.

The above considerations suggest that the phase space for the gauged $\mathrm{SL}(2, \mathbb{R}) \mathrm{WZW}$ model, subject to the boundary conditions (2.19), (2.32), (2.34) and (2.47) is made up of the symplectic leaves, $\Gamma_{\bar{P}, P, B}$, labeled by the real numbers $B(2.48)$ as well as $P, \bar{P}$, which satisfy the relation $P-\bar{P}=\mathrm{k}+$ w with $\mathrm{w} \in \mathbb{Z}$. On each leaf the symplectic form is non-degenerate. The algebra of functions on the leaf, $\Gamma_{\bar{P}, P, B}^{\star}$, is generated by the currents $W_{j}^{(c l)}(u)$ and $\bar{W}_{j}^{(c l)}(\bar{u})$, subject to the reality and boundary conditions $(2.54),(2.55)$, which form the classical $\bar{W}_{\infty} \otimes W_{\infty}$ - algebra

There are two evident continuous symmetries. The first one is the $\mathrm{U}(1)$ invariance w.r.t. a shift of the compact variable $\alpha(2.28)$,

$$
\mathcal{U}_{\phi}: \quad \mathrm{e}^{\mathrm{i} \alpha \sigma^{y}} \mapsto \mathrm{e}^{\mathrm{i}(\alpha+\phi) \sigma^{y}} .
$$

The other one corresponds to the transformation

$$
\mathcal{R}_{\theta}: \quad \eta(t, x) \mapsto \eta(t, x)+\theta .
$$

Both symmetry transformations are canonical, i.e., they preserve the symplectic structure. They have no effect on the $W$ currents and act trivially on the symplectic leaves. The gauged $\mathrm{SL}(2, \mathbb{R})$ WZW model also possesses the global symmetries, which leave the Lagrange density (2.13), the constraint (2.14) as well as the boundary conditions imposed on the fields unchanged. We'll use the notation $\mathcal{D}$ for the $\mathcal{Z}_{2}$ symmetry, which acts on the fields and the symplectic leaves as

$$
\begin{aligned}
\mathcal{D}: \quad \mathbf{G} & \mapsto-\sigma^{y} \mathbf{G} \sigma^{y}, \quad \eta \rightarrow-\eta \\
\Gamma_{\bar{P}, P, B} & \mapsto \Gamma_{\bar{P}, P, B^{-1}} .
\end{aligned}
$$


Also, by $\mathcal{C P}$ symmetry, we'll mean the invariance under the transformation

$$
\mathcal{C P}: \quad \mathbf{G}(t, x) \mapsto(\mathbf{G}(t,-x))^{-1}, \quad \eta(t, x) \mapsto \eta(t,-x) .
$$

Note that the condition $P-\bar{P}=\mathrm{k}+\mathrm{w} \quad(\mathrm{w} \in \mathbb{Z})$ is unchanged when $P \mapsto-\bar{P}, \bar{P} \mapsto-P$. This suggests that the action of $\mathcal{C P}$ on the symplectic leaves is described as

$$
\mathcal{C P}: \Gamma_{\bar{P}, P, B} \mapsto \Gamma_{-P,-\bar{P}, B}
$$

\section{Quantum gauged $\mathrm{SL}(2, \mathbb{R}) \mathrm{WZW}$ model}

\subsection{BRST quantization}

Once the gauged $\mathrm{SL}(2, \mathbb{R})$ WZW model is formulated as a classical dynamical system possessing constraints of the first class one can consider the problem of its quantization within the BRST approach. Here we briefly sketch the algebraic procedure for the construction of the chiral component of the space of states.

The chiral component of the energy momentum tensor of the quantum theory is split into three terms:

$$
T_{\text {total }}=T_{\mathrm{WZW}}+T_{\mathrm{Gauss}}+T_{\text {ghost }} .
$$

The first one is [18]

$$
T_{\mathrm{wZW}}=-\frac{n^{2}}{n+C_{\mathrm{V}}} \kappa_{A B} L^{A} L^{B} .
$$

It is built from the left currents of the WZW model

$$
L^{A}(u)=n^{-1} \sum_{m=-\infty}^{\infty} j_{m}^{A} \mathrm{e}^{-\mathrm{i} m u} \quad(A=3, \pm)
$$

whose Fourier coefficients obey the commutation relations

$$
\left[j_{m}^{A}, j_{r}^{B}\right]=-n \kappa^{A B} \frac{m}{2} \delta_{m+r, 0}-\frac{\mathrm{i}}{2} f_{C}^{A B} j_{m+r}^{C} .
$$

Here the level (central element) of the Kac-Moody algebra has been denoted by $n$. It is related to the Plank constant as

$$
\hbar=\frac{2 \pi}{n} .
$$

The constant $C_{\mathrm{V}}$ entering into (3.2) stands for the so-called dual Coxeter number:

$$
C_{\mathrm{V}} \kappa^{A B}=\frac{1}{4} f_{D}^{A C} f^{B D}
$$

and in the case under consideration $C_{\mathrm{V}}=2$. The second term in (3.1) represents the contribution of the massless Gaussian field,

$$
T_{\text {Gauss }}=n(\partial \eta)^{2}
$$

with

$$
\partial \eta(u)=n^{-\frac{1}{2}} \sum_{m=-\infty}^{\infty} a_{m} \mathrm{e}^{-\mathrm{i} m u}: \quad\left[a_{m}, a_{r}\right]=\frac{m}{2} \delta_{m+r, 0}
$$


Finally $T_{\text {ghost }}$ is the chiral component of the energy momentum tensor for the $b c$-system:

$$
T_{\text {ghost }}=\mathrm{i} b \partial c .
$$

The ghost fields have conformal dimensions $\left(\Delta_{b}, \Delta_{c}\right)=(1,0)$ and, as with the chiral fields $L^{A}$ and $\partial \eta$, can also be expanded in the Fourier series

$$
b(u)=\sum_{m=-\infty}^{\infty} b_{m} \mathrm{e}^{-\mathrm{i} m u}, \quad c(u)=\sum_{m=-\infty}^{\infty} c_{m} \mathrm{e}^{-\mathrm{i} m u},
$$

where

$$
\left\{b_{m}, c_{r}\right\}=\delta_{m+r, 0}, \quad\left\{b_{m}, b_{r}\right\}=\left\{c_{m}, c_{r}\right\}=0 .
$$

The Virasoro central charge of the $b c$-system is equal to $(-2)$, so that the total central charge associated with the energy momentum tensor (3.1) is given by

$$
c_{\text {total }}=c_{\mathrm{WZW}}+c_{\mathrm{Gauss}}+c_{\mathrm{ghost}}=\frac{3 n}{n+2}+1-2=2-\frac{6}{n+2} .
$$

The highest weight representation for the combined chiral algebra generated by the Fourier coefficients $j_{m}^{A}, a_{m}, b_{m}, c_{m}$ is constructed in the usual manner. First of all one requires that the highest state is annihilated by all the positive frequency modes with $m>0$. Since the zero modes of the WZW currents satisfy the commutation relations

$$
\left[j_{0}^{A}, j_{0}^{B}\right]=-\frac{\mathrm{i}}{2} f^{A B}{ }_{C} j_{0}^{C},
$$

the highest states form a representation of the $\mathfrak{s l}_{2}$ algebra. It makes sense to require that it is an irreducible one, characterized by the value of the Casimir operator

$$
\hat{C}_{\mathrm{G}}=-\kappa_{A B} j_{0}^{A} j_{0}^{B},
$$

which in the $\mathfrak{s l}_{2}$ case is usually denoted as $\ell(\ell+1)$. Rather than $\ell$ we will employ the parameter $p=\ell+\frac{1}{2}$. Together with this quantum number the highest states can be labeled by the eigenvalues of the zero modes $j_{0}^{3}$ and $a_{0}$ :

$$
\hat{C}_{\mathrm{G}}|p, \mu, s\rangle=\left(p^{2}-\frac{1}{4}\right)|p, \mu, s\rangle, \quad j_{0}^{3}|p, \mu, s\rangle=\mu|p, \mu, s\rangle, \quad a_{0}|p, \mu, s\rangle=\frac{s}{\sqrt{n}}|p, \mu, s\rangle .
$$

The highest states form a representation not only of the $\mathfrak{s l}_{2}$ algebra but also the simple fermionic one

$$
\left\{b_{0}, c_{0}\right\}=1, \quad b_{0}^{2}=c_{0}^{2}=0 .
$$

Thus we supplement the set of conditions defining them with

$$
c_{0}|p, \mu, s\rangle_{+}=0, \quad|p, \mu, s\rangle_{-} \equiv b_{0}|p, \mu, s\rangle_{+} .
$$

The highest weight representation is built by the action of the negative frequency modes $j_{m}^{A}, a_{m}, b_{m}, c_{m}$ with $m<0$ on the highest state multiplet. The corresponding linear space will be denoted by $\mathcal{A}_{p, s}$. The latter possesses a grading induced by the Virasoro algebra 
generator $L_{0}^{(\text {total })}$. For given $\mathrm{L}=0,1,2, \ldots$, the level subspace $\mathcal{A}_{p, s}^{(\mathrm{L})}$ is finite dimensional and all its states have the same conformal dimension $\Delta_{p, s}+\mathrm{L}$ with

$$
\Delta_{p, s}=\frac{p^{2}-\frac{1}{4}}{n+2}+\frac{s^{2}}{n} .
$$

Note that the conformal dimensions of the primary states do not depend on the quantum number $\mu$.

The parameter $p$ and its barred counterpart $\bar{p}$ are related to the central elements (2.20)(2.22) of the Poisson algebra of the WZW currents. In particular the sum $p+\bar{p}$ can be identified with the eigenvalues of the operator $-\mathrm{i} \frac{\partial}{\partial \alpha}$ with $\alpha$ being the dynamical variable from (2.28). Then the compactness condition $\alpha \sim \alpha+2 \pi$ yields the quantization rule $p+\bar{p} \in \mathbb{Z}$. This, in view of the classical relation $(2.30)$, leads to ${ }^{4}$

$$
p=\frac{1}{2}(\mathrm{u}+(n+2)(\mathrm{k}+\mathrm{w})), \quad \bar{p}=\frac{1}{2}(\mathrm{u}-(n+2)(\mathrm{k}+\mathrm{w})) \quad(\mathrm{u}, \mathrm{w} \in \mathbb{Z}) .
$$

At the same time $s$ may take any real value,

$$
s \in \mathbb{R} .
$$

The central rôle in the BRST approach belongs to the BRST charge and the ghost number operator. These obey the relations

$$
\hat{Q}_{\mathrm{BRST}}^{2}=0, \quad\left[\hat{Q}_{\mathrm{BRST}}, \hat{q}_{\text {ghost }}\right]=\hat{Q}_{\mathrm{BRST}} .
$$

In the case at hand they read explicitly as

$$
\begin{aligned}
\hat{Q}_{\mathrm{BRST}} & =\frac{1}{\hbar} \int_{0}^{2 \pi} \mathrm{d} u\left(L^{3}-\partial \eta\right) c(u)=\left(j_{0}^{3}-\sqrt{n} a_{0}\right) c_{0}+\sum_{m \neq 0}\left(j_{m}^{3}-\sqrt{n} a_{m}\right) c_{-m} \\
\hat{q}_{\mathrm{ghost}} & =\int_{0}^{2 \pi} \frac{\mathrm{d} u}{2 \pi} b c(u)=b_{0} c_{0}+\sum_{m=1}^{\infty}\left(b_{-m} c_{m}-c_{-m} b_{m}\right) .
\end{aligned}
$$

It is easy to see that both operators commute with the zero mode of the current $L^{3}(u)$ :

$$
\left[j_{0}^{3}, \hat{Q}_{\text {BRST }}\right]=\left[j_{0}^{3}, \hat{q}_{\text {ghost }}\right]=0 .
$$

Let $\mu$ be an eigenvalue of $j_{0}^{3}$ corresponding to one of the states from the highest state multiplet of $\mathcal{A}_{p, s}$. Consider the eigenspace $\mathcal{A}_{p, \mu, s} \subset \mathcal{A}_{p, s}$ such that

$$
j_{0}^{3} \mathcal{A}_{p, \mu, s}=\mu \mathcal{A}_{p, \mu, s} .
$$

Since both the BRST charge and ghost number operator act invariantly in this subspace, one can introduce the component of the factor space $\operatorname{Ker}\left[Q_{\mathrm{BRST}}\right] / \operatorname{Im}\left[Q_{\mathrm{BRST}}\right]$ with zero ghost number,

$$
\widetilde{\mathcal{A}}_{p, \mu, s} \subset \operatorname{Ker}\left[Q_{\mathrm{BRST}}\right] /\left.\operatorname{Im}\left[Q_{\mathrm{BRST}}\right]\right|_{\mathcal{A}_{p, \mu, s}}: \quad \hat{q}_{\text {ghost }} \tilde{\mathcal{A}}_{p, \mu, s}=0 .
$$

\footnotetext{
${ }^{4}$ Here we identify the difference $p-\bar{p}$ with $(n+2)(P-\bar{P})$. Within the semi-classical analysis where $n \gg 1, n$ and $n+2$ are indistinguishable. The finite renormalization $n \mapsto n+2$ may be advocated for using similar arguments as those in the work [6].
} 
The operators $\hat{Q}_{\mathrm{BRST}}, \hat{q}_{\text {ghost }}$ and $j_{0}^{3}$, all commute with the energy momentum tensor

$$
\left[\hat{Q}_{\mathrm{BRST}}, T_{\text {total }}(u)\right]=\left[\hat{q}_{\mathrm{ghost}}, T_{\text {total }}(u)\right]=\left[j_{0}^{3}, T_{\text {total }}(u)\right]=0 .
$$

This implies that $\widetilde{\mathcal{A}}_{p, \mu, s}$ is a naturally graded space and similar to $\mathcal{A}_{p, s}$ admits the decomposition

$$
\widetilde{\mathcal{A}}_{p, \mu, s}=\bigoplus_{\mathrm{L}=0}^{\infty} \widetilde{\mathcal{A}}_{p, \mu, s}^{(\mathrm{L})}
$$

The dimensions of the level subspaces $\widetilde{\mathcal{A}}_{p, \mu, s}^{(\mathrm{L})}$ depend essentially on whether or not $\mu-s$ vanishes. This difference is the coefficient in front of the ghost zero mode $c_{0}$ in (3.21) when the action of the BRST charge is restricted to the eigenspace $\mathcal{A}_{p, \mu, s}$. Consider the highest states $|p, \mu, s\rangle_{ \pm}$. If $\mu \neq s$, then the state $|p, \mu, s\rangle_{+}$is annihilated by the BRST charge. On the other hand $\hat{Q}_{\mathrm{BRST}}|p, \mu, s\rangle_{-} \neq 0$ and is proportional to $|p, \mu, s\rangle_{+}$. This implies that the level subspace $\widetilde{\mathcal{A}}_{p, \mu, s}^{(0)}$ with $\mu \neq s$ is trivial. In the case when $\mu=s$ both highest states are annihilated by the BRST charge. However only $|p, s, s\rangle_{+}$has zero ghost number so that $\operatorname{dim}\left(\widetilde{\mathcal{A}}_{p, s, s}^{(0)}\right)=1$. Recall that $|p, s, s\rangle_{+}$is a state from a $\mathfrak{s l}_{2}$ irrep characterized by $p$. The eigenvalues of $j_{0}^{3}$ for the other highest states from the multiplet are given by $\mu=s+\mathrm{i} m$, where $m$ is a nonzero integer, and hence the difference $\mu-s$ for these states would be nonvanishing. Proceeding further, it is straightforward to check at least for small values of $\mathrm{L}=0,1,2, \ldots$, that all the spaces $\widetilde{\mathcal{A}}_{p, \mu, s}^{\mathrm{L})}$ are trivial for $\mu \neq s$, while the dimensions of $\widetilde{\mathcal{A}}_{p, s, s}^{(\mathrm{L})}$ with generic $p$ is equal to the number of bipartitions of $\mathrm{L}$.

\section{$3.2 W_{\infty}$-algebra}

Perhaps the easiest way to explore the linear structure of the factor space $\widetilde{\mathcal{A}}_{p, \mu, s}$ is to bosonize the $\widehat{\mathfrak{s l}}(2, \mathbb{R})$ current algebra [19-21]. This allows one to isolate the physical states in $\mathcal{A}_{p, s}$ and to show that $\operatorname{dim}\left(\widetilde{\mathcal{A}}_{p, s, s}^{(\mathrm{L})}\right)$ coincides with the corresponding dimensions of the level subspaces of the highest weight representation of the quantum $W_{\infty}$-algebra. The bosonization of $\widehat{\mathfrak{s l}}(2, \mathbb{R})$ requires three chiral Bose fields. The spin 2 current which commutes with $L^{3}(u)$ is identified with $W_{2}(u)$. It can be arranged so that

$$
W_{2}=(\partial \vartheta)^{2}+(\partial \varphi)^{2}+\frac{\mathrm{i}}{\sqrt{n+2}} \partial^{2} \varphi
$$

which involves only two of the chiral fields. By construction the field $W_{2}$ commutes with the BRST charge and $\hat{q}_{\text {ghost }}$. Using the Operator Product Expansion (OPE),

$$
\varphi(u) \varphi(0)=-\frac{1}{2} \log (u)+O(1), \quad \vartheta(u) \vartheta(0)=-\frac{1}{2} \log (u)+O(1),
$$

a simple calculation shows that

$$
W_{2}(u) W_{2}(0)=\frac{c}{2 u^{4}}-\frac{2}{u^{2}} W_{2}(0)-\frac{1}{u} \partial W_{2}(0)+O(1),
$$

where the central charge

$$
c=2-\frac{6}{n+2}
$$


coincides with $c_{\text {total }}$ from eq. (3.11). The spin $3 W$ current is also obtained from the commutativity condition with $L^{3}(u)$. However, it contains an ambiguity related to the addition of $\partial W_{2}(u)$. The requirement that $W_{3}(u)$ be a primary conformal field, i.e.,

$$
W_{2}(u) W_{3}(0)=-\frac{3}{u^{2}} W_{3}(0)-\frac{1}{u} \partial W_{3}(0)+O(1)
$$

fixes it up to an overall multiplicative factor. We take

$$
W_{3}=\frac{6 n+8}{3 n+6}(\partial \vartheta)^{3}+2(\partial \varphi)^{2} \partial \vartheta+\mathrm{i} \sqrt{n+2} \partial^{2} \varphi \partial \vartheta-\frac{\mathrm{i} n}{\sqrt{n+2}} \partial \varphi \partial^{2} \vartheta+\frac{n}{6(n+2)} \partial^{3} \vartheta .
$$

As in the classical case, the currents $W_{2}$ and $W_{3}$ generate a closed algebra, whose commutation relations may be conveniently encoded via OPEs. In particular, the spin 4 current is obtained via the OPE of $W_{3}$ with itself:

$$
\begin{aligned}
& W_{3}(u) W_{3}(0)=-\frac{c(c+7)(2 c-1)}{9(c-2) u^{6}}+\frac{(c+7)(2 c-1)}{3(c-2) u^{4}}\left(W_{2}(u)+W_{2}(0)\right)-\frac{1}{u^{2}} \\
& \times\left(W_{4}(u)+W_{4}(0)+W_{2}^{2}(u)+W_{2}^{2}(0)+\frac{2 c^{2}+22 c-25}{30(c-2)}\left(\partial^{2} W_{2}(u)+\partial^{2} W_{2}(0)\right)\right)+O(1) .
\end{aligned}
$$

The definition of $W_{4}$, in principle, is not unique and contains the freedom in the addition of any of the spin 4 fields $\partial^{2} W_{2}, \partial W_{3}$ as well as the composite field $W_{2}^{2}$. Fixing $W_{4}$ as in eq. (3.33), it turns out that the singular terms $\propto u^{-6}$ and $u^{-3}$ are absent in the OPE

$$
W_{2}(u) W_{4}(0)=\frac{(c+10)(17 c+2)}{15(c-2) u^{4}} W_{2}(0)-\frac{4}{u^{2}} W_{4}(0)-\frac{1}{u} \partial W_{4}(0)+O(1) .
$$

This way, by recursively computing OPEs one can generate the full set of quantum $W$ currents $\left\{W_{j}(u)\right\}_{j=2}^{\infty}$. In the classical limit with $n \rightarrow \infty$,

$$
W_{j} \rightarrow n^{j / 2} W_{j}^{(c l)}
$$

and the quantum $W_{\infty}$-algebra becomes the classical Poisson algebra, whose first few PB relations are given in (2.56) [17].

The quantum $W$ currents, similar to the classical ones, are periodic fields and can be expanded in a Fourier series,

$$
W_{j}(u)=-\frac{c}{24} \delta_{j, 2}+\sum_{m=-\infty}^{\infty} \widetilde{W}_{j}(m) \mathrm{e}^{-\mathrm{i} m u} .
$$

Note that the expansion coefficients for the $W_{2}$ current, defined in such a way, satisfy the Virasoro algebra commutation relations:

$$
\left[L_{m}, L_{r}\right]=(m-r) L_{m+r}+\frac{c}{12} m\left(m^{2}-1\right) \delta_{m+r, 0} \quad\left(L_{m} \equiv \widetilde{W}_{2}(m)\right) .
$$

The Fourier modes $\widetilde{W}_{j}(m)$ are used in the construction of the highest weight irreps of the $W_{\infty}$-algebra. The highest state is defined by the conditions

$$
\widetilde{W}_{j}(m)|\boldsymbol{\omega}\rangle=0 \quad(\forall m>0), \quad \widetilde{W}_{j}(0)|\boldsymbol{\omega}\rangle=\omega_{j}|\boldsymbol{\omega}\rangle,
$$


where $\boldsymbol{\omega}=\left(\omega_{2}, \omega_{3}\right)$ is the highest weight. Notice that the component $\omega_{2}$ is equal to the conformal dimension of the state. It turns out that $|\boldsymbol{\omega}\rangle$ is fully specified by the relations (3.38) with $j=2,3$. The linear span of states

$$
\widetilde{W}_{2}\left(-l_{1}\right) \ldots \widetilde{W}_{2}\left(-l_{m}\right) \widetilde{W}_{3}\left(-l_{1}^{\prime}\right) \ldots \widetilde{W}_{3}\left(-l_{m^{\prime}}^{\prime}\right)|\boldsymbol{\omega}\rangle
$$

with $1 \leq l_{1} \leq l_{2} \leq \ldots \leq l_{m}$ and $1 \leq l_{1}^{\prime} \leq l_{2}^{\prime} \leq \ldots \leq l_{m^{\prime}}^{\prime}$ form a representation of the $W_{\infty}$ algebra which is usually referred to as the Verma module. The latter is a graded linear space, and the dimensions of its level subspace with $\mathrm{L}=\sum_{i} l_{i}+\sum_{i^{\prime}} l_{i^{\prime}}^{\prime}$ is given by $\operatorname{par}_{2}(\mathrm{~L})$ the number of bipartitions of L. For generic values of the highest weight $\boldsymbol{\omega}$ and the central charge $c$, the Verma module is an irrep of the $W_{\infty}$ - algebra. However, under certain conditions imposed on the parameters, it may contain null vectors - highest states occurring at non-zero levels. In this case the irrep can be obtained from the Verma module by factoring out all of the invariant subspace(s) generated by the null vector(s). We'll parameterize the central charge by $n$ according to (3.30), and the highest weight $\boldsymbol{\omega}$ by the pair $(p, s)$ as

$$
\begin{aligned}
& \omega_{2}=\frac{p^{2}-\frac{1}{4}}{n+2}+\frac{s^{2}}{n} \equiv \Delta_{p, s} \\
& \omega_{3}=\frac{2 s}{\sqrt{n}}\left(\frac{p^{2}}{n+2}+\frac{(3 n+4) s^{2}}{3 n(n+2)}-\frac{2 n+3}{12(n+2)}\right) .
\end{aligned}
$$

In turn the highest weight irrep of the $W_{\infty}$-algebra will be denoted by $\mathcal{W}_{p, s} \equiv \mathcal{W}_{-p, s}$.

The motivation for swapping from $\boldsymbol{\omega}$ to the pair of parameters $(p, s)$ is based on the following. The bosonization formulae (3.27) and (3.32) introduce the structure of the $W_{\infty}$ Verma module in the Fock space $\mathcal{F}_{\mathbf{P}}$. The latter is the space of the representation of two independent copies of the Heisenberg algebra, generated by the Fourier modes of $\partial \vartheta$ and $\partial \varphi$, whose commutation relations are the same as in eq. (3.8). The space $\mathcal{F}_{\mathbf{P}}$ with $\mathbf{P}=\left(\frac{p}{\sqrt{n+2}}, \frac{s}{\sqrt{n}}\right)$ is characterized by the highest weight - the values of the zero modes of the chiral fields $\partial \vartheta, \partial \varphi$ in $\mathcal{F}_{\mathbf{P}}$ :

$$
\left.\int_{0}^{2 \pi} \frac{\mathrm{d} u}{2 \pi} \partial \varphi\right|_{\mathcal{F}_{\mathbf{P}}}=\frac{p}{\sqrt{n+2}},\left.\quad \quad \int_{0}^{2 \pi} \frac{\mathrm{d} u}{2 \pi} \partial \vartheta\right|_{\mathcal{F}_{\mathbf{P}}}=\frac{s}{\sqrt{n}} .
$$

Then eqs. (3.27) and (3.32) yield that the highest weight $\boldsymbol{\omega}=\left(\omega_{2}, \omega_{3}\right)$ is related to $p$ and $s$ as in (3.40).

\subsection{Space of states}

So far we have been mainly focused on just one chirality. Of course, all the above follows through for the other chirality as well. This way one concludes that the algebra of extended conformal symmetry for the gauged $\operatorname{SL}(2, \mathbb{R})$ WZW model coincides with $\bar{W}_{\infty} \otimes W_{\infty}$. In turn the full space of states, $\mathcal{H}^{\text {(cont) }}$, can be decomposed into the irreps $\overline{\mathcal{W}}_{\bar{p}, s} \otimes \mathcal{W}_{p, s}$ of this algebra. At this point we put forward the conjecture that for generic values of the twist parameter $\mathrm{k}$, the linear structure of $\mathcal{H}^{(\mathrm{cont})}$ is described by

$$
\mathcal{H}^{(\text {cont })}=\bigoplus_{\mathrm{u}, \mathrm{w} \in \mathbb{Z}} \int_{\mathbb{R}}^{\oplus} \mathrm{d} s \overline{\mathcal{W}}_{\bar{p}, s} \otimes \mathcal{W}_{p, s} \quad \text { with } \quad \begin{aligned}
p & =\frac{1}{2} \mathrm{u}+\frac{1}{2}(n+2)(\mathrm{k}+\mathrm{w}) \\
\bar{p} & =\frac{1}{2} \mathrm{u}-\frac{1}{2}(n+2)(\mathrm{k}+\mathrm{w}) .
\end{aligned}
$$


The subscript "cont" is used to emphasize the presence of the direct integral in the above linear decomposition.

Let's clarify the condition that $-\frac{1}{2}<\mathrm{k} \leq \frac{1}{2}$ be generic. What we mean by this is that $(n+2) \mathrm{k} \notin \mathbb{Z}$. Then, if $s$ is real, each of the irreps $\mathcal{W}_{p, s}$ and $\overline{\mathcal{W}}_{\bar{p}, s}$ coincide with the Verma module. The corresponding character,

$$
\operatorname{ch}_{p, s}(\mathbf{q}) \equiv \operatorname{Tr}_{\mathcal{W}_{p, s}}\left[\mathrm{q}^{\widetilde{W}_{2}(0)-\frac{c}{24}}\right]
$$

is given by

$$
\operatorname{ch}_{p, s}(\mathrm{q})=\frac{\mathrm{q}^{-\frac{1}{12}+\frac{s^{2}}{n}+\frac{p^{2}}{n+2}}}{(\mathrm{q}, \mathrm{q})_{\infty}^{2}} \quad(p, s \text { generic })
$$

where $(\mathrm{q}, \mathrm{q})_{\infty}^{-2}$ is the generating function for $\operatorname{par}_{2}(\mathrm{~L})$ :

$$
\frac{1}{(\mathrm{q}, \mathrm{q})_{\infty}^{2}} \equiv \prod_{m=1}^{\infty} \frac{1}{\left(1-\mathrm{q}^{m}\right)^{2}}=\sum_{\mathrm{L}=0}^{\infty} \operatorname{par}_{2}(\mathrm{~L}) \mathrm{q}^{\mathrm{L}}
$$

Each term in the linear decomposition (3.42) can be interpreted as being the result of the quantization of the algebra of functions on the classical symplectic leaf $\Gamma_{\bar{P}, P, B}$. One can make the following identification

$$
B=\mathrm{e}^{\frac{4 \pi s}{n}},
$$

while in the classical limit $\frac{\bar{p}}{n+2} \rightarrow \bar{P}, \frac{p}{n+2} \rightarrow P$. The classical theory possesses a set of global symmetries, which were described in eqs. (2.58)-(2.62) and are inherited by the quantum model. Regarding the continuous symmetries, the operators $\hat{\mathcal{U}}_{\phi}$ and $\hat{\mathcal{R}}_{\theta}$, being restricted to a $\bar{W}_{\infty} \otimes W_{\infty}$ irrep, are proportional to the identity operator:

$$
\begin{aligned}
& \hat{\mathcal{U}}_{\phi}\left(\overline{\mathcal{W}}_{\bar{p}, s} \otimes \mathcal{W}_{p, s}\right)=\mathrm{e}^{\mathrm{i}(\bar{p}+p) \phi} \overline{\mathcal{W}}_{\bar{p}, s} \otimes \mathcal{W}_{p, s} \\
& \hat{\mathcal{R}}_{\theta}\left(\overline{\mathcal{W}}_{\bar{p}, s} \otimes \mathcal{W}_{p, s}\right)=\mathrm{e}^{2 \mathrm{i} s \theta} \overline{\mathcal{W}}_{\bar{p}, s} \otimes \mathcal{W}_{p, s} .
\end{aligned}
$$

The generator of the $\mathcal{Z}_{2}$ symmetry, $\hat{\mathcal{D}}$, acts as the intertwiner,

$$
\hat{\mathcal{D}}: \overline{\mathcal{W}}_{\bar{p}, s} \otimes \mathcal{W}_{p, s} \mapsto \overline{\mathcal{W}}_{\bar{p},-s} \otimes \mathcal{W}_{p,-s}
$$

and satisfies the following relations with the $W$ currents

$$
\hat{\mathcal{D}} W_{j}(u) \hat{\mathcal{D}}=(-1)^{j} W_{j}(u), \quad \hat{\mathcal{D}} \bar{W}_{j}(\bar{u}) \hat{\mathcal{D}}=(-1)^{j} \bar{W}_{j}(\bar{u}) .
$$

Similarly, the action of the $\mathcal{C P}$ transformation is described by the formula

$$
\mathcal{C P}: \quad \overline{\mathcal{W}}_{\bar{p}, s} \otimes \mathcal{W}_{p, s} \mapsto \overline{\mathcal{W}}_{-p, s} \otimes \mathcal{W}_{-\bar{p}, s}, \quad \hat{\mathcal{C}} \hat{\mathcal{P}} W_{j}(u)=\bar{W}_{j}(u) \hat{\mathcal{C}} \hat{\mathcal{P}} .
$$

In the classical theory the $W$ currents are real fields (see eq. (2.54)). Upon quantization, this translates to the conjugation conditions

$$
\left(W_{j}(u)\right)^{\star}=W_{j}\left(u^{*}\right), \quad\left(\bar{W}_{j}(\bar{u})\right)^{\star}=\bar{W}_{j}\left(\bar{u}^{*}\right) .
$$


The latter induce an inner product for the states belonging to the irreps $\overline{\mathcal{W}}_{\bar{p}, s} \otimes \mathcal{W}_{p, s}$. In the case when the central charge $c<2$ it turns out that such an inner product is not a positive definite one [5]. This way the space of states $\mathcal{H}^{(\mathrm{cont})}$ is equipped with the structure of a pseudo-Hilbert space. The conjugation conditions (3.51) are not sufficient to fix the inner product of the $\bar{W}_{\infty} \otimes W_{\infty}$ primary states, $\Psi_{\mathrm{u}, \mathrm{w}, \mathrm{s}}$, which, in general, has the form

$$
\left\langle\Psi_{\mathrm{u}^{\prime}, \mathrm{w}^{\prime}, s^{\prime}}, \Psi_{\mathrm{u}, \mathrm{w}, s}\right\rangle=N_{\mathrm{u}, \mathrm{w}, s} \delta_{\mathrm{u}^{\prime}, \mathrm{u}} \delta_{\mathrm{w}^{\prime}, \mathrm{w}} \delta\left(s^{\prime}-s\right) .
$$

Notice that, though the coefficients $N_{\mathrm{u}, \mathrm{w}, \mathrm{s}}$ contain the ambiguity

$$
N_{\mathrm{u}, \mathrm{w}, \mathrm{s}} \mapsto N_{\mathrm{u}, \mathrm{w}, \mathrm{s}}\left|C_{\mathrm{u}, \mathrm{w}, s}\right|^{2},
$$

a change in the normalization of the primary states has no effect on the sign of $N_{\mathrm{u}, \mathrm{w}, s}$ for given $\mathrm{u}$, w and $s$. Thus, despite the ambiguity, the "norms" $N_{\mathrm{u}, \mathrm{w}, \mathrm{s}}$ are an important characteristic of the quantum theory.

\section{Lorentzian black hole NLSM}

\subsection{Space of states}

The field theory governed by the action (1.3a) with periodic boundary conditions imposed on $U$ and $V$ corresponds to the gauged WZW model with $\mathrm{k}=0$. However, as was already pointed out, even at the classical level this case requires some special attention. A brief examination of formula (3.42) specialized to $\mathrm{k}=0$ shows that each term $\overline{\mathcal{W}}_{\bar{p}, s} \otimes \mathcal{W}_{p, s}$ in the decomposition of $\mathcal{H}^{\text {(cont) }}$ appears together with $\overline{\mathcal{W}}_{-\bar{p}, s} \otimes \mathcal{W}_{-p, s}$. Both of them are equivalent representations of the $\bar{W}_{\infty} \otimes W_{\infty}$-algebra. This signalizes the presence of an additional global symmetry that would commute with all of the $W$ currents

$$
\hat{\mathcal{C}} W_{j}(u)=W_{j}(u) \hat{\mathcal{C}}, \quad \hat{\mathcal{C}} \bar{W}_{j}(\bar{u})=\bar{W}_{j}(\bar{u}) \hat{\mathcal{C}} .
$$

We will refer to it as $\mathcal{C}$ conjugation. The space of states $\mathcal{H}^{(\text {cont })}$ is splitted into the two components

$$
\mathcal{H}^{(\text {cont })}=\mathcal{H}_{\text {even }}^{(\text {cont })} \bigoplus \mathcal{H}_{\text {odd }}^{(\text {cont })}
$$

having definite $\mathcal{C}$ parity +1 (even) and -1 (odd). As linear spaces, each of the components are identical. ${ }^{5}$ Together with the $W$ currents, $\hat{\mathcal{C}}$ also commutes with the symmetry operators $\hat{\mathcal{D}}, \hat{\mathcal{C}} \hat{\mathcal{P}}$ and $\hat{\mathcal{R}}_{\theta}$ :

$$
[\hat{\mathcal{C}}, \hat{\mathcal{D}}]=[\hat{\mathcal{C}}, \hat{\mathcal{C}} \hat{\mathcal{P}}]=\left[\hat{\mathcal{C}}, \hat{\mathcal{R}}_{\theta}\right]=0
$$

However with $\hat{\mathcal{U}}_{\phi}$ it satisfies the relation

$$
\hat{\mathcal{C}} \hat{\mathcal{U}}_{\phi} \hat{\mathcal{C}}=\hat{\mathcal{U}}_{-\phi}
$$

Hence, since $\phi \sim \phi+2 \pi$, the operator $\hat{\mathcal{C}}$ commutes only with $\hat{\mathcal{U}}_{\pi}$ - the generator of a $180^{\circ}$ rotation.

\footnotetext{
${ }^{5}$ The space $\overline{\mathcal{W}}_{\bar{p}, s} \otimes \mathcal{W}_{p, s}$ with $p=-\bar{p}=\frac{1}{2}(n+2)$ w turns out to be $\mathcal{C}$ invariant and the splitting into its $\mathcal{C}$ even and $\mathcal{C}$ odd components requires some clarification. This will be addressed in section 6 .
} 
At the classical level, the action of the $\hat{\mathcal{U}}_{\pi}$ transformation, as follows from the general formula (2.58) and (2.28), reduces to flipping the sign of the matrix $\mathbf{G}$ :

$$
\begin{aligned}
\mathcal{U}_{\pi}: \mathbf{G}_{\bar{P}, P, B} & \mapsto-\mathbf{G}, \quad \eta \Gamma_{\bar{P}, P, B} . &
\end{aligned}
$$

At the same time, the action of the $\mathcal{C}$ conjugation can be identified as

$$
\begin{aligned}
\mathcal{C}: \quad \mathbf{G} & \mapsto-\sigma^{z} \mathbf{G} \sigma^{z}, \quad \eta \mapsto \eta \\
\Gamma_{\bar{P}, P, B} & \mapsto \Gamma_{-\bar{P},-P, B}
\end{aligned}
$$

which is similar to the relation (2.60) for the $\mathcal{Z}_{2}$ symmetry transformation $\mathcal{D}{ }^{6}$ Recall that applying the gauge fixing condition $X=Y$ (see (2.12)) in the original formulation of the gauged $\mathrm{SL}(2, \mathbb{R})$ WZW model, results in two identical copies of the Lorentzian black hole NLSM corresponding to the cases $X=Y>0$ and $X=Y<0$. The $\mathcal{C}$ conjugation intertwines these two copies. By "symmetrizing" them one can arrange so that $\mathcal{C}$, by definition, would act as the identity operator in the Lorentzian black hole NLSM.

To get more insight into the symmetries and the global structure of the phase space of the classical model, it is useful to consider elementary solutions of the classical equations of motion. These may be constructed by setting $f=\bar{f}=0$ as well as $\xi_{ \pm}=P$ and $\bar{\xi}_{ \pm}=\bar{P}$ in the general relations (2.42)-(2.45), yielding

$$
U(t, x)=V(t, x)=\sin ((P+\bar{P}) t+(P-\bar{P}) x) .
$$

One can easily see that the equations of motion corresponding to the action (1.3a) are indeed satisfied. The periodic boundary condition for $U$ and $V$ requires that the difference $P-\bar{P}$ be an integer,

$$
P-\bar{P}=0, \pm 1, \pm 2, \ldots,
$$

which coincides with (2.30) for the case $\mathrm{k}=0$.

The explicit solutions provide some hints concerning the action of the global symmetries on the phase space. There are two evident space-time symmetry transformations of the model (1.3a). Namely, time-reversal and parity conjugation, which are defined as

$$
\begin{aligned}
& \mathcal{T}: \quad U(t, x) \mapsto U(-t, x), \quad V(t, x) \mapsto \quad V(-t, x) \\
& \mathcal{P}: \quad U(t, x) \mapsto-U(t,-x), \quad V(t, x) \mapsto-V(t,-x) .
\end{aligned}
$$

The extra sign in the definition of $\mathcal{P}$ is a matter of convention since the transformation $\mathcal{U}_{\pi}$ : $U \mapsto-U, V \mapsto-V$ is also a symmetry. The solutions (4.7) are unchanged under the $\mathcal{P} \mathcal{T}$ transformation. More generally, we will assume that two solutions related via $\mathcal{P} \mathcal{T}$ belong to the same symplectic leaf, i.e.,

$$
\mathcal{P} \mathcal{T}: \quad \Gamma_{\bar{P}, P, B} \mapsto \Gamma_{\bar{P}, P, B} .
$$

The action of $\mathcal{P}$ and $\mathcal{T}$ on the fundamental fields, as described by formula (4.9), induces the action of these transformations on $\Gamma_{\bar{P}, P, B}$. We make the assumption that two solutions

\footnotetext{
${ }^{6}$ For $\mathrm{k} \neq 0$ the $\mathcal{C}$ invariance of the model is broken by the condition (2.34).
} 
related through separate $\mathcal{P}$ or $\mathcal{T}$ transformations belong to different symplectic leaves. A brief examination of (4.7) motivates that

$$
\begin{array}{ll}
\mathcal{T}: & \Gamma_{\bar{P}, P, B} \mapsto \Gamma_{-P,-\bar{P}, B} \\
\mathcal{P}: & \Gamma_{\bar{P}, P, B} \mapsto \Gamma_{P, \bar{P}, B} .
\end{array}
$$

An immediate consequence is that $\mathcal{P} \mathcal{T}$ maps $\Gamma_{\bar{P}, P, B}$ to $\Gamma_{-\bar{P},-P, B}$. In view of the condition (4.10) the following identification must be made

$$
\Gamma_{\bar{P}, P, B} \equiv \Gamma_{-\bar{P},-P, B}
$$

Notice that the latter is required for setting the $\mathcal{C}$ conjugation (4.6) to be the identity transformation for the Lorentzian black hole NLSM. Then, without loss of generality, one can assume that

$$
P+\bar{P} \geq 0 \text {. }
$$

It should be emphasized that the identification (4.12) is expected to hold true only in the case $\mathrm{k}=0$, as otherwise neither $\mathcal{P}$ nor $\mathcal{C}$ separately are symmetries of the model.

Taking into account (4.13) one arrives at the conjecture that the space of states of the Lorentzian black hole NLSM is given by

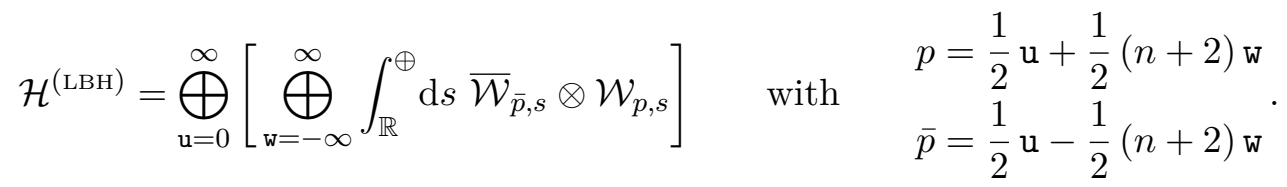

At first glance, in view of the above discussion of the $\mathcal{C}$ conjugation, the formula seems to be a straightforward consequence of (3.42). However, one should keep in mind that the notation $\mathcal{W}_{p, s}$ stands for the irreducible highest weight representation of the $W_{\infty}$-algebra. For $(n+2) \mathrm{k} \notin \mathbb{Z}$ and $s \in \mathbb{R}$ each of the irreps $\mathcal{W}_{\bar{p}, s}$ and $\mathcal{W}_{p, s}$ coincides with the Verma module. However for $\mathrm{k}=0$ some of the Verma modules become reducible. It turns out that (see, e.g., $[22,32]$ )

$$
\mathcal{V}_{e r_{\rho, s}}=\mathcal{W}_{\rho, s} \bigoplus \mathcal{W}_{\rho+m(n+2), s} \quad \text { for } \quad \rho=\frac{1}{2}(r-m(n+2)), \quad m, r=1,2, \ldots
$$

Note that the character of $\mathcal{W}_{\rho, s}$ in the direct sum reads as

$$
\begin{array}{rlrl}
\operatorname{ch}_{\rho, s}(\mathrm{q})=\mathrm{q}^{-\frac{1}{12}+\frac{s^{2}}{n}+\frac{\rho^{2}}{n+2}} \frac{1-\mathrm{q}^{m r}}{(\mathrm{q}, \mathrm{q})_{\infty}^{2}}, & \rho=\frac{1}{2}(r-m(n+2)), \quad m, r=1,2, \ldots \\
s \in \mathbb{R}, \quad n-\text { generic },
\end{array}
$$

while the character of $\mathcal{W}_{\rho+m(n+2), s}$ is described by eq. (3.44). This way the $\mathcal{C}$ even component of the space (3.42) as $\mathrm{k} \rightarrow 0$ admits the decomposition

$$
\mathcal{H}_{\text {even }}^{(\text {cont })}=\mathcal{H}^{(\mathrm{LBH})} \bigoplus \mathcal{H}^{(\text {null })} .
$$

Here $\mathcal{H}^{(\mathrm{LBH})}$ is given by $(4.14)$, while the space $\mathcal{H}^{(\text {null) }}$ is a direct sum of two components,

$$
\mathcal{H}^{(\text {null })}=\mathcal{H}_{+}^{(\text {null })} \bigoplus \mathcal{H}_{-}^{(\text {null })} .
$$


The latter are decomposed identically into the irreps of the algebra of extended conformal symmetry

$$
\mathcal{H}_{ \pm}^{(\mathrm{null})}=\bigoplus_{\mathrm{v}, \mathrm{w}=1}^{+\infty} \int_{\mathbb{R}}^{\oplus} \mathrm{d} s \overline{\mathcal{W}}_{\rho, s} \otimes \mathcal{W}_{\rho, s} \quad\left(\rho=\frac{1}{2} \mathrm{v}+\frac{1}{2}(n+2) \mathrm{w}\right)
$$

The highest state in either one $\overline{\mathcal{W}}_{\rho, s}$ or $\mathcal{W}_{\rho, s}$, appearing in the integrand, coincides with the null vector in the original Verma module (see (4.15)).

There is a simple minded argument in support of the dropping of the "null" component in (4.17) for the construction of the space of states of the Lorentzian black hole NLSM. The space $\mathcal{H}^{\text {(null) }}$ admits an evident symmetry, which interchanges the subspaces $\mathcal{H}_{+}^{\text {(null) }}$ and $\mathcal{H}_{-}^{(\text {null })}$ in (4.18). On the other hand, there is no clear manifestation of such an additional $\mathcal{Z}_{2}$ symmetry for the model described by the classical action (1.3a).

\subsection{Minisuperspace approximation}

For a better qualitative understanding of the quantum NLSM it is useful to consider the model within the so-called minisuperspace approximation. This entails taking into account only those field configurations that do not depend on the space co-ordinate $x$, such as the classical solutions (4.7) with $P=\bar{P}$. We still keep $U$ and $V$ to satisfy the constraint $0 \leq U V<1$ corresponding to the union of regions III and IV in figure 1. For a preliminary analysis it is convenient to parameterize $U, V$ from this domain as

$$
U=\mathrm{e}^{\Theta} \sin (\Phi), \quad V=\mathrm{e}^{-\Theta} \sin (\Phi) ; \quad \Phi \in\left(-\frac{\pi}{2}, \frac{\pi}{2}\right), \quad \Theta \in(-\infty, \infty) .
$$

Then the minisuperspace version of the classical action (1.3a) reads as

$$
S_{\mathrm{LBH}}^{(\mathrm{ms})}=\frac{\pi}{\hbar} \int \mathrm{d} t\left(\dot{\Phi}^{2}-\tan ^{2}(\Phi) \dot{\Theta}^{2}\right)
$$

Since the generalized coordinate $\Theta$ is cyclic, its conjugate momentum $\Pi_{\Theta}=-\tan ^{2}(\Phi) \dot{\Theta}$ is an integral of motion. The effective Lagrangian (the Routhian) for the non-cyclic degree of freedom is given by

$$
L_{\mathrm{eff}}=\frac{1}{2}\left(\dot{\Phi}^{2}-V_{\mathrm{eff}}(\Phi)\right), \quad V_{\mathrm{eff}}(\Phi)=-\Pi_{\Theta}^{2} \cot ^{2}(\Phi) .
$$

The latter describes a $1 \mathrm{D}$ particle falling to the origin $\Phi=0$. An elementary calculation shows that for any value $\Pi_{\Theta} \neq 0$ the particle, starting its motion at $t=0$, reaches the origin in a finite amount of time $t_{\text {fall }}<+\infty$. For $t>t_{\text {fall }}$ the motion remains undetermined. Thus the action (4.21) specifies the time evolution of the mechanical system only within a finite time interval (except for the trajectories with $\Pi_{\Theta}=0$ ). To continue the classical trajectories for $t>t_{\text {fall }}$ the unbounded effective potential should be somehow regularized. There are of course numerous ways of doing this. A simple minded one is to replace $V_{\text {eff }}(\Phi)=-\Pi_{\Theta}^{2} \cot ^{2}(\Phi)$ by a smooth potential $V_{\text {eff }}^{(\mathrm{reg})}(\Phi)$, which together with its derivative is bounded from below within the infinitesimal interval $\Phi \in(-\epsilon, \epsilon)$. Outside this interval 
$V_{\text {eff }}^{(\text {reg })}(\Phi)=V_{\text {eff }}(\Phi)$. To keep the original symmetry of the potential we assume that the regularized one is an even function:

$$
V_{\text {eff }}^{(\text {reg })}(\Phi)=V_{\text {eff }}^{(\text {reg })}(-\Phi)
$$

Then the motion of $\Phi$ becomes globally defined and periodic for any values of $\Pi_{\Theta} \neq 0$.

With basic intuition from quantum mechanics, we can predict the symmetry properties of the minisuperspace stationary wave functions. First of all, that the regularized potential is an even function of $\Phi$ implies that the stationary states may be assigned a parity $\sigma= \pm 1$,

$$
\hat{\mathcal{U}}_{\pi} \Psi^{(\sigma)}(U, V) \equiv \Psi^{(\sigma)}(-U,-V)=\sigma \Psi^{(\sigma)}(U, V),
$$

where we now switch to the original target space coordinates $(U, V)$. This relates the values of the wave function in the domains III and IV from figure 1 . Next, $\Psi^{(\sigma)}$ can be chosen to be an eigenfunction of the operator $\hat{\Pi}_{\Theta}=\frac{\hbar}{\mathrm{i}} \partial_{\Theta}=\frac{\hbar}{\mathrm{i}}\left(U \partial_{U}-V \partial_{V}\right)$ :

$$
\hat{\Pi}_{\Theta} \Psi_{s}^{(\sigma)}=2 \hbar s \Psi_{s}^{(\sigma)} .
$$

It follows that

$$
\Psi_{s}^{(\sigma)}(U, V)=\left(\frac{U}{V}\right)^{\text {is }} F_{s}^{(\sigma)}(U V)
$$

The minisuperspace approximation ignores the presence of the oscillatory modes so that the wave functions $\Psi_{s}^{(\sigma)}$ are expected to correspond to $\bar{W}_{\infty} \otimes W_{\infty}$ primary states, characterized by $p=\bar{p}$ and $s$ (it is instructive to compare eqs. (4.24) and (4.25) with (3.47a) and (3.47b), respectively). In turn the minisuperspace energy becomes $\Delta_{p, s}+\Delta_{\bar{p}, s}=2 \Delta_{p, s}$ in the leading non-vanishing order of $\hbar=\frac{2 \pi}{n}$ (the approximation is reliable only in the limit $n \rightarrow \infty$ ). Namely,

$$
E^{(\mathrm{ms})}=\frac{\hbar}{\pi}\left(p^{2}+s^{2}-\frac{1}{4}\right) .
$$

At this point $p$ can be thought of as a real number parameterizing the minisuperspace energy $E^{(\mathrm{ms})}$ and the corresponding wavefunction $\Psi_{p, s}^{(\sigma)}$. Since the highest weight is an even function of $p$,

$$
\Psi_{-p, s}^{(\sigma)}(U, V)=\Psi_{p, s}^{(\sigma)}(U, V) .
$$

As was emphasized previously, one can assume that $p=\bar{p} \geq 0$.

Though the highest weight of the $W_{\infty}$ irrep $\boldsymbol{\omega}=\left(\omega_{2}, \omega_{3}\right)$ is not sensitive to the sign of $p$, as follows from (3.40) it does depend on the sign of $s: \omega_{3}(p,-s)=-\omega_{3}(p, s)$. Thus the primary states characterized by $(p, s)$ and $(p,-s)$ are distinguishable. They are related through the $\mathcal{Z}_{2}$ transformation, so that

$$
\hat{\mathcal{D}} \Psi_{p, s}^{(\sigma)}(U, V)=\Psi_{p,-s}^{(\sigma)}(U, V) .
$$

On the other hand, by definition, this symmetry interchanges $U$ and $V$ :

$$
\hat{\mathcal{D}} \Psi_{p, s}^{(\sigma)}(U, V) \equiv \Psi_{p, s}^{(\sigma)}(V, U) .
$$


Combining the above two relations with (4.26) one concludes that

$$
\Psi_{p, s}^{(\sigma)}(U, V)=\left(\frac{U}{V}\right)^{\mathrm{i} s} F_{p, s}^{(\sigma)}(U V), \quad \text { where } \quad F_{p, s}^{(\sigma)}(z)=F_{-p, s}^{(\sigma)}(z)=F_{p,-s}^{(\sigma)}(z)
$$

Having described the symmetry properties of the stationary wave functions, we turn to deriving them explicitly. In the work [6], a minisuperspace analysis was performed for the $\operatorname{NLSM}(1.3 \mathrm{a})$ with the fields $U, V$ belonging to region I from figure 1 (or equivalently II). Though this is not the domain of interest, we can still follow the same line of arguments of that paper. In particular, up to a trivial factor, the minisuperspace Hamiltonian coincides with the "dilatonic" Laplacian:

$$
\hat{H}^{(\mathrm{ms})}=-\frac{\hbar}{4 \pi} \triangle_{D}, \quad \triangle_{D}=\frac{1}{\mathrm{e}^{D} \sqrt{-G}} \partial_{i}\left(\mathrm{e}^{D} \sqrt{-G} G^{i j} \partial_{j}\right),
$$

where the metric is the one in (1.1) and the dilaton field is given by

$$
D=\log (1-U V) .
$$

The stationary Schrödinger equation $\hat{H}^{(\mathrm{ms})} \Psi=E^{(\mathrm{ms})} \Psi$ reads explicitly as

$$
-\left((1-U V) \partial_{U} \partial_{V}-\frac{1}{2}\left(U \partial_{U}+V \partial_{V}\right)\right) \Psi=\frac{\pi}{\hbar} E^{(\mathrm{ms})} \Psi
$$

Using the general form (4.26) for the stationary wave functions and parameterizing the energy as in (4.27), it is straightforward to show that $F(z)=z^{-\mathrm{is}} F_{p, s}^{(\sigma)}(z)$ obeys the Gauss hypergeometric equation

$$
z(1-z) F^{\prime \prime}+(1+2 \mathrm{i} s-2(1+\mathrm{i} s) z) F^{\prime}-\left(\frac{1}{2}+\mathrm{i} s+p\right)\left(\frac{1}{2}+\mathrm{i} s-p\right) F=0 .
$$

Keeping in mind our preliminary analysis, the ODE (4.35) is applicable only in the domain $\epsilon^{2}<z<1$ with a small regularization parameter $\epsilon \ll 1$ (recall that $z=U V=\sin ^{2}(\Phi)$ ).

The function $F_{p, s}^{(\sigma)}(z)$ (4.31) is a certain linear combination of $z^{ \pm \mathrm{i} s}{ }_{2} F_{1}\left(\frac{1}{2} \pm \mathrm{i} s+p, \frac{1}{2} \pm\right.$ is $-p, 1 \pm 2 \mathrm{i} s, z)$, which can be specified as follows. Applying the elementary identity

$$
\mathrm{e}^{D} \sqrt{-G}\left(\Psi_{1}^{*} \hat{H}^{(\mathrm{ms})} \Psi_{2}-\Psi_{2} \hat{H}^{(\mathrm{ms})} \Psi_{1}^{*}\right)=\frac{\hbar}{4 \pi} \partial_{i}\left[\mathrm{e}^{D} \sqrt{-G} G^{i j}\left(\Psi_{2} \partial_{j} \Psi_{1}^{*}-\Psi_{1}^{*} \partial_{j} \Psi_{2}\right)\right]
$$

to the pair of stationary wave functions $\Psi_{1}, \Psi_{2}$ corresponding to the energies $E_{1}^{(\mathrm{ms})}, E_{2}^{(\mathrm{ms})}$ and then integrating the result over the domain $\mathrm{B}_{\epsilon}: \epsilon^{2}<U V<1$, one obtains

$$
\left(E_{2}^{(\mathrm{ms})}-E_{1}^{(\mathrm{ms})}\right) \int_{\mathrm{B}_{\epsilon}} \mathrm{d} U \mathrm{~d} V \mathrm{e}^{D} \sqrt{-G} \Psi_{1}^{*} \Psi_{2}=\frac{\hbar}{4 \pi} \int_{\partial \mathrm{B}_{\epsilon}} \mathrm{d} \ell \mathrm{e}^{D}\left(\Psi_{2} \partial_{n} \Psi_{1}^{*}-\Psi_{1}^{*} \partial_{n} \Psi_{2}\right) .
$$

Here the integral in the r.h.s. is taken over the boundary of $\mathrm{B}_{\epsilon}$, which is the union of $U V=\epsilon^{2}$ and $U V=1$. Also, $\partial_{n}$ stands for the normal derivative to $\partial \mathrm{B}_{\epsilon}$. As was discussed before, the wave functions possess a definite parity. Due to this either the wave function or its normal derivative vanishes at $U V=0$. Hence as $\epsilon \rightarrow 0$ the horizon $U V=0$ does not contribute to the r.h.s. of eq. (4.37). Further, since the dilaton factor $\mathrm{e}^{D}$ vanishes 
at the black hole singularity $U V=1$ one could make the whole boundary integral vanish by imposing that both the eigenfunctions and their normal derivatives remain finite at $U V=1$. In this case the wave functions corresponding to different energies would be orthogonal w.r.t. the inner product

$$
\left\langle\Psi_{1}, \Psi_{2}\right\rangle=\int_{0<U V<1} \mathrm{~d} U \mathrm{~d} V \mathrm{e}^{D} \sqrt{-G} \Psi_{1}^{*} \Psi_{2} .
$$

This suggests to take $F_{p, s}^{(\sigma)}(z)$ in $(4.31)$ as

$$
F_{p, s}^{(\sigma)}(z)=z_{2}^{\mathrm{i} s} F_{1}\left(\frac{1}{2}+\mathrm{i} s+p, \frac{1}{2}+\mathrm{i} s-p, 1 ; 1-z\right) \quad\left(\epsilon^{2}<z<1\right)
$$

or, equivalently,

$$
\begin{aligned}
F_{p, s}^{(\sigma)}(z)= & A_{p,+s} z^{+\mathrm{i} s}{ }_{2} F_{1}\left(\frac{1}{2}+\mathrm{i} s+p, \frac{1}{2}+\mathrm{i} s-p, 1+2 \mathrm{i} s ; z\right) \\
& +A_{p,-s} z^{-\mathrm{i} s}{ }_{2} F_{1}\left(\frac{1}{2}-\mathrm{i} s+p, \frac{1}{2}-\mathrm{i} s-p, 1-2 \mathrm{i} s ; z\right),
\end{aligned}
$$

where

$$
A_{p, s}=\frac{\Gamma(-2 \mathrm{i} s)}{\Gamma\left(\frac{1}{2}-\mathrm{i} s-p\right) \Gamma\left(\frac{1}{2}-\mathrm{i} s+p\right)} .
$$

For $z \ll 1$ it is convenient to use the variable $y$ such that $z=\mathrm{e}^{y}$. Then $F_{p, s}^{(\sigma)}$ asymptotically approaches to a superposition of two plane waves

$$
F_{p, s}^{(\sigma)} \asymp A_{p,+s} \mathrm{e}^{+\mathrm{i} s y}+A_{p,-s} \mathrm{e}^{-\mathrm{i} s y} \quad(1 \ll(-y)<2 \log (1 / \epsilon)) .
$$

The regularized interaction discussed before in the domain $(-y)>2 \log (1 / \epsilon)$ results in a quantization condition for $s$

$$
\epsilon^{-4 \mathrm{is} s} \mathrm{e}^{\frac{\mathrm{i}}{2} \delta^{(\mathrm{ms})}(p, s)} \asymp \sigma .
$$

The phase shift $\delta^{(\mathrm{ms})}$ here depends on the precise form of the regularized potential. As $\epsilon \rightarrow 0$, the spectrum of $s$ becomes continuous and is characterized by the density of states

$$
\rho^{(\mathrm{ms})}(s)=\frac{2}{\pi} \log (1 / \epsilon)+\frac{1}{4 \pi} \partial_{s} \delta^{(\mathrm{ms})}(p, s) .
$$

The corresponding minisuperspace wave functions would be orthogonal w.r.t. the inner product (4.38):

$$
\left\langle\Psi_{p^{\prime}, s^{\prime}}^{\left(\sigma^{\prime}\right)}, \Psi_{p, s}^{(\sigma)}\right\rangle \propto \delta_{p^{\prime}, p} \delta_{\sigma^{\prime}, \sigma} \delta\left(s^{\prime}-s\right) .
$$

Here we use the Dirac $\delta$-function for $s$ since the latter can be any real number. At the same time the Kronecker symbol indicates that $p$ belongs to some discrete set. The quantization of $p$ seems rather natural once we note that the term $\frac{\hbar}{\pi}\left(p^{2}-\frac{1}{4}\right)$ in the formula for the minisuperspace energy (4.27) can be interpreted as the contribution of the non-cyclic degree of freedom $\Phi$, which executes periodic motion in the regularized effective potential. This is consistent with our discussion of the quantization of the Lorentzian black hole NLSM. Setting $\mathrm{w}=0$ in formula (4.14) giving the admissible values of $p$ and $\bar{p}$, one has $2 p=2 \bar{p}=$ $\mathrm{v}=0,1,2, \ldots$ Also $\delta_{\sigma^{\prime}, \sigma}$ in (4.45) can be ignored - the sign factor $\sigma$ is not an independent quantum number and is defined by the parity of the integer $\mathrm{v}$ (see eq. (3.47a) with $\phi=\pi$ ). 


\section{Low energy states of the $\mathcal{Z}_{2}$ invariant spin chain in the scaling limit. Continuous spectrum}

In the seminal work [23], Baxter introduced a multiparametric, integrable, statistical system that covers a variety of classes of critical behaviour. In particular, it was observed in [24] that the $\mathcal{Z}_{2}$ invariant spin chain, corresponding to a certain specialization of the parameters of the general Baxter model, is critical and possesses a continuous spectrum of scaling dimensions. The spin chain was subsequently studied in the works [25, 27-31]. In the recent paper [32] a systematic analysis, including a study of the finite size corrections, was performed. Arguments were presented that the low energy states, in a suitably defined scaling limit, organize into the space $\mathcal{H}^{(\text {cont })}$, whose linear structure is described by eq. (3.42). This leads to the idea that the critical behaviour of the spin chain is governed by the gauged WZW model. Here, accepting the conjecture, we use the results obtained for the lattice system to move forward in the study of the field theory.

\subsection{Global symmetries and Hermitian structure}

The subject of our interest is a spin $\frac{1}{2}$ chain of length $N$, which is always an even number, governed by the Hamiltonian ${ }^{7}$

$$
\begin{aligned}
\mathbb{H}=\frac{1}{\sin (2 \gamma)} \sum_{m=1}^{N}( & 2 \sin ^{2}(\gamma) \sigma_{m}^{z} \sigma_{m+1}^{z}-\left(\sigma_{m}^{x} \sigma_{m+2}^{x}+\sigma_{m}^{y} \sigma_{m+2}^{y}+\sigma_{m}^{z} \sigma_{m+2}^{z}\right) \\
& \left.\quad-\mathrm{i} \sin (\gamma)\left(\sigma_{m}^{x} \sigma_{m+1}^{x}+\sigma_{m}^{y} \sigma_{m+1}^{y}\right)\left(\sigma_{m-1}^{z}-\sigma_{m+2}^{z}\right)\right)+N \cot (2 \gamma) \hat{\mathbf{1}} .
\end{aligned}
$$

The operators $\sigma_{m}^{A}$ stand for the Pauli matrices that act non-trivially in the $m$-th factor of the tensor product

$$
\mathscr{V}_{N}=\mathbb{C}_{N}^{2} \otimes \mathbb{C}_{N-1}^{2} \otimes \cdots \otimes \mathbb{C}_{1}^{2} \quad(N-\text { even }) .
$$

They are taken to satisfy the quasiperiodic boundary conditions:

$$
\sigma_{N+\ell}^{ \pm}=\mathrm{e}^{ \pm 2 \mathrm{i} \pi \mathrm{k}} \sigma_{\ell}^{ \pm}, \quad \sigma_{N+\ell}^{z}=\sigma_{\ell}^{z} \quad(\ell=1,2),
$$

where

$$
\sigma_{m}^{ \pm}=\frac{1}{2}\left(\sigma_{m}^{x} \pm \mathrm{i} \sigma_{m}^{y}\right)
$$

The Hamiltonian commutes with the $z$ projection of the total spin operator

$$
\mathbb{S}^{z}=\frac{1}{2} \sum_{m=1}^{N} \sigma_{m}^{z}: \quad\left[\mathbb{S}^{z}, \mathbb{H}\right]=0,
$$

which is the infinitesimal generator of the $\mathrm{U}(1)$ symmetry. The action of the finite rotation $\hat{\mathcal{U}}_{\phi}$ on the local spin operators is given by

$$
\hat{\mathcal{U}}_{\phi} \sigma_{m}^{ \pm} \hat{\mathcal{U}}_{\phi}^{-1}=\mathrm{e}^{ \pm \mathrm{i} \phi} \sigma_{m}^{ \pm}, \quad \hat{\mathcal{U}}_{\phi} \sigma_{m}^{z} \hat{\mathcal{U}}_{\phi}^{-1}=\sigma_{m}^{z} .
$$

\footnotetext{
${ }^{7}$ This form for the Hamiltonian, up to an overall multiplicative factor and an additive constant, appeared in ref. [26]. The one defined by eq. (2) in the work [31] coincides with $\hat{V} \mathbb{H} \hat{V}^{-1}$, where $\mathbb{H}$ is as in (5.1), while $\hat{\mathrm{V}}=\prod_{m=1}^{N / 2} \exp \left(\frac{\mathrm{i} \pi}{4} \sigma_{2 m-1}^{z}\right)$.
} 
Another evident symmetry of (5.1) and (5.3) is $\mathcal{C} \mathcal{P}$-invariance. The corresponding transformation is described through the formula

$$
\hat{\mathcal{C}} \hat{\mathcal{P}} \sigma_{m}^{ \pm} \hat{\mathcal{C}} \hat{\mathcal{P}}=\sigma_{N+1-m}^{\mp}, \quad \hat{\mathcal{C}} \hat{\mathcal{P}} \sigma_{m}^{z} \hat{\mathcal{C}} \hat{\mathcal{P}}=-\sigma_{N+1-m}^{z} \quad(m=1, \ldots, N) .
$$

A characteristic property of the model is the presence of an additional $\mathcal{Z}_{2}$ symmetry. The adjoint action of its generator $\hat{\mathcal{D}}$ on $\sigma_{m}^{A}$ is more involved and for odd $m$ reads as

$$
\begin{aligned}
& \hat{\mathcal{D}} \sigma_{m}^{ \pm} \hat{\mathcal{D}}=\frac{1}{\cos (\gamma)}\left(\sigma_{m+1}^{ \pm}-\mathrm{i} \sin (\gamma) \sigma_{m+1}^{z} \sigma_{m}^{ \pm}\right) \\
& \hat{\mathcal{D}} \sigma_{m}^{z} \hat{\mathcal{D}}=\frac{1}{\cos ^{2}(\gamma)}\left(\sigma_{m+1}^{z}-\sin ^{2}(\gamma) \sigma_{m}^{z}+2 \mathrm{i} \sin (\gamma)\left(\sigma_{m+1}^{+} \sigma_{m}^{-}+\sigma_{m+1}^{-} \sigma_{m}^{+}\right)\right),
\end{aligned}
$$

while for even $m$ :

$$
\begin{aligned}
& \hat{\mathcal{D}} \sigma_{m}^{ \pm} \hat{\mathcal{D}}=\frac{1}{\cos (\gamma)}\left(\sigma_{m-1}^{ \pm}+\mathrm{i} \sin (\gamma) \sigma_{m}^{ \pm} \sigma_{m-1}^{z}\right) \\
& \hat{\mathcal{D}} \sigma_{m}^{z} \hat{\mathcal{D}}=\frac{1}{\cos ^{2}(\gamma)}\left(\sigma_{m-1}^{z}-\sin ^{2}(\gamma) \sigma_{m}^{z}-2 \mathrm{i} \sin (\gamma)\left(\sigma_{m}^{-} \sigma_{m-1}^{+}+\sigma_{m}^{+} \sigma_{m-1}^{-}\right)\right) .
\end{aligned}
$$

The lattice system also possesses the time-reversal symmetry generated by the anti-unitary transformation $\hat{\mathcal{T}}$, such that

$$
\hat{\mathcal{T}} \boldsymbol{\Psi}=\left(\prod_{m=1}^{N} \sigma_{m}^{x}\right) \boldsymbol{\Psi}^{*}, \quad \boldsymbol{\Psi} \in \mathscr{V}_{N} .
$$

With the anisotropy parameter in the domain $0<\gamma<\pi$, the spin chain (5.1), (5.3) is critical. However, different types of critical behaviour occur depending on whether $\gamma \in\left(0, \frac{\pi}{2}\right)$ or $\gamma \in\left(\frac{\pi}{2}, \pi\right)$. The latter case was considered in ref. [26]. The relation between the spin chain and the gauged WZW model, which was proposed in the work [32], occurs when $0<\gamma<\frac{\pi}{2}$. Then $\gamma$ is related to the parameter $n \equiv 2 \pi / \hbar$ from the field theory side as

$$
\gamma=\frac{\pi}{n+2} \quad(0<n<+\infty) .
$$

The twist parameter $\mathrm{k} \in\left(-\frac{1}{2}, \frac{1}{2}\right]$ in (5.3) is identified with that entering into the boundary condition (2.32).

The Hamiltonian (5.1) is not Hermitian w.r.t. the usual matrix Hermitian conjugation $\mathcal{O}^{\dagger}=\left(\mathcal{O}^{T}\right)^{*}$. Nevertheless one can introduce a conjugation,

$$
\hat{\mathrm{O}}^{\star}=\hat{\mathrm{X}}_{\star}^{-1} \hat{\mathrm{O}}^{\dagger} \hat{\mathrm{X}}_{\star}
$$

for which the Hamiltonian satisfies

$$
\mathbb{H}^{\star}=\mathbb{H} .
$$

The expression for the matrix $\hat{\mathrm{X}}_{\star}=\hat{\mathrm{X}}_{\star}^{\dagger}$ is given by formula (19.62) in [32]. It should be kept in mind that the $\star$-conjugation does not correspond to any positive definite inner product. A manifestation of this is that some of the eigenvalues of the $2^{N}$ dimensional matrix $\mathbb{H}$ are complex. The analysis of [32] shows that the conjugation (5.11) in the scaling limit induces 
the field theory conjugation for the $W$ currents (3.51) in the space $\mathcal{H}^{(\text {cont) }}$. Moreover it yields that the normalization of the $W_{\infty} \otimes W_{\infty}$ primary states can be chosen such that their "norms", i.e., the coefficients $N_{\mathrm{u}, \mathrm{w}, s}$ entering into eq. (3.52), are given by

$$
N_{\mathrm{u}, \mathrm{w}, \mathrm{s}}=\frac{\Gamma\left(1+\frac{2 \bar{p}}{n+2}\right) \Gamma\left(1+\frac{2 p}{n+2}\right)}{\Gamma(1+2 \bar{p}) \Gamma(1+2 p)} \quad \text { with } \quad \begin{aligned}
p & =\frac{1}{2} \mathrm{u}+\frac{1}{2}(n+2)(\mathrm{k}+\mathrm{w}) \\
\bar{p} & =\frac{1}{2} \mathrm{u}-\frac{1}{2}(n+2)(\mathrm{k}+\mathrm{w}) .
\end{aligned}
$$

Note that the norms are independent of $s$, while the quantum number $\mathrm{u}$ is identified with the eigenvalue of the operator $\mathbb{S}^{z}$ :

$$
\mathrm{u}=S^{z}=0, \pm 1, \pm 2, \ldots
$$

Similar to the gauged WZW model with $\mathrm{k}=0$, the spin chain subject to periodic boundary conditions possesses an extra symmetry - that of $\mathcal{C}$ conjugation. For the finite lattice system the matrix $\hat{\mathcal{C}}$ is given by

$$
\hat{\mathcal{C}}=c_{N} \prod_{m=1}^{N}\left(\eta_{m}\right)^{\frac{1}{2} \sigma_{m}^{z}} \sigma_{m}^{x}, \quad \text { where } \quad \eta_{m}=(-1)^{m+1} \mathrm{i}
$$

and the choice of the overall sign factor, $c_{N}^{2}=1$, is a matter of convention. ${ }^{8}$ Since $\hat{\mathcal{C}}$ anticommutes with the $z$ projection of the total spin,

$$
\hat{\mathcal{C}} \mathbb{S}^{z}=-\mathbb{S}^{z} \hat{\mathcal{C}}
$$

the $\mathcal{C}$ even and $\mathcal{C}$ odd components of the space of states of the spin chain would not be invariant w.r.t. the action of the $\mathrm{U}(1)$ transformation (5.6), except the case $\phi=\pi$. Nevertheless $\left|S^{z}\right|=0,1,2, \ldots$ is a well defined quantum number for the states from each component. Taking the scaling limit results in the spaces $\mathcal{H}_{\text {even }}^{\text {(cont) }}$ and $\mathcal{H}_{\text {odd }}^{\text {(cont) }}$, which appear in eq. (4.2). Recall that of special interest is the subspace $\mathcal{H}^{(\mathrm{LBH})}(4.14)$ of $\mathcal{H}_{\text {even }}^{\text {(cont) }}$, which is expected to serve as the space of states for the Lorentzian black hole NLSM. The Hermitian structure of $\mathcal{H}^{(\mathrm{LBH})}$ is specified through the conjugation conditions (3.51) of the $W$ currents as well as the norms of the primary $W_{\infty} \otimes W_{\infty}$ states occurring in its decomposition. The latter may be obtained from (5.13) via a taking of the limit $\mathrm{k} \rightarrow 0$. However, special care is needed for the states with $u=0$ and $w \neq 0$, as the $\Gamma$-functions in that formula become singular. The issue is treated in the work [32] with the result that

$$
\left\langle\Psi_{\mathrm{u}^{\prime}, \mathrm{w}^{\prime}, s^{\prime}}, \Psi_{\mathrm{u}, \mathrm{w}, s}\right\rangle_{\mathrm{LBH}}=N_{\mathrm{u}, \mathrm{w}}^{(\mathrm{LBH})} \delta_{\mathrm{u}^{\prime}, \mathrm{u}} \delta_{\mathrm{w}^{\prime}, \mathrm{w}} \delta\left(s^{\prime}-s\right),
$$

${ }^{8} \mathrm{We}$ found it convenient to set

$$
c_{N}=\left\{\begin{array}{ll}
(-1)^{N / 4} & N / 2-\text { even } \\
1 & N / 2-\text { odd }
\end{array} .\right.
$$

For $N / 2$ even the ground state, i.e., the state with the lowest possible energy (ordered w.r.t. the real part), of the lattice Hamiltonian with periodic boundary conditions is non-degenerate. With this choice of $c_{N}$ its $\mathcal{C}$ parity is equal to +1 . When $N / 2$ is odd there are two ground states, forming a $\mathcal{Z}_{2}$ doublet, which are distinguished by their $\mathcal{C}$ parity. 
where

$$
N_{0,0}^{(\mathrm{LBH})}=1, \quad N_{\mathrm{u}, \mathrm{w}}^{(\mathrm{LBH})}= \begin{cases}(-1)^{\mathrm{w}} \frac{\sin (\pi(n+2) \mathrm{w})}{\pi(n+2)} & \mathrm{u}=0, \mathrm{w} \neq 0 \\ \frac{\Gamma\left(1-\mathrm{w}+\frac{\mathrm{u}}{n+2}\right) \Gamma\left(1+\mathrm{w}+\frac{\mathrm{u}}{n+2}\right)}{\Gamma(1+\mathrm{u}-(n+2) \mathrm{w}) \Gamma(1+\mathrm{u}+(n+2) \mathrm{w})} & \mathrm{u} \geq 1, \mathrm{w} \in \mathbb{Z}\end{cases}
$$

Then $\mathcal{H}^{(\mathrm{LBH})}$ is a pseudo-Hilbert space equipped with a non-positive definite inner product. This would reflect the fact that the target space for the NLSM (1.3a) has Lorentzian signature.

\subsection{Density of states}

The distinguishing feature of the spin chain (5.1), (5.3) in the parametric domain $0<$ $\gamma<\frac{\pi}{2}$ is that the spectrum of the rescaled energy $\left(\mathcal{E}-\mathcal{E}_{\text {vac }}\right) N$, though it remains discrete for finite $N$, becomes densely distributed as $N \rightarrow \infty$. The analysis of the scaling limit leads to a certain density matrix for the gauged $\mathrm{SL}(2, \mathbb{R})$ WZW model as well as the Lorentzian black hole NLSM. Here we give a brief summary of the relevant results obtained in refs. [24, 25, 27-32].

A key rôle in the description of the scaling limit of the $\mathcal{Z}_{2}$ invariant spin chain belongs to the so-called quasi-shift operator $\mathbb{B}$. The latter was first introduced in ref. [27]. The expression for this operator, in the conventions adopted in this paper, is given by eq. (8.4) of the work [32]. The quasi-shift operator belongs to the commuting family of matrices, which includes the Hamiltonian $\mathbb{H}$, the $z$ projection of the total spin $\mathbb{S}^{z}$ and the lattice translation operator $\mathbb{K}$, whose matrix elements read as

$$
(\mathbb{K})_{a_{N} a_{N-1} \ldots a_{1}}^{b_{N} b_{N-1} \ldots b_{1}}=\mathrm{e}^{\mathrm{i} \pi \mathrm{k}\left(a_{1}+a_{2}\right)} \delta_{a_{N}}^{b_{N-2}} \delta_{a_{N-1}}^{b_{N-3}} \ldots \delta_{a_{1}}^{b_{N-1}} .
$$

Here the indices $a_{m}$ and $b_{m}$ take the values \pm and label the states in the space (5.2).

Each eigenstate belonging to the low energy part of the spectrum can be assigned, together with $S^{z}$, the "winding number" $\mathrm{w}=0, \pm 1, \pm 2, \ldots$ and a pair of non-negative integers $(\overline{\mathrm{L}}, \mathrm{L})$, which are referred to as the levels. In view of the conjectured relation with the gauged WZW model, we swap the notation $S^{z}$ in favour of u (see (5.14)) and also use $p$ and $\bar{p}$ defined through eq. (3.18). The extensive numerical work performed in refs. [24, 25, 27-31] suggests that the large $N$ behaviour of the eigenvalues of the Hamiltonian $\mathbb{H}$ and the lattice translation operator $\mathbb{K}$ is described by the formula

$$
\begin{aligned}
\mathcal{E} & =e_{\infty} N+\frac{4 \pi v_{\mathrm{F}}}{N}\left(\frac{p^{2}+\bar{p}^{2}}{n+2}+\frac{2 b^{2}}{n}-\frac{1}{6}+\mathrm{L}+\overline{\mathrm{L}}\right)+o\left(N^{-1-\varepsilon}\right) \\
K & =\exp \left(\frac{4 \pi \mathrm{i}}{N}\left(\frac{p^{2}-\bar{p}^{2}}{n+2}+\mathrm{L}-\overline{\mathrm{L}}\right)\right) .
\end{aligned}
$$

Here

$$
e_{\infty}=-\frac{2 v_{\mathrm{F}}}{\pi} \int_{0}^{\infty} \mathrm{d} t \frac{\sinh \left(\frac{2 t}{n}\right)}{\sinh \left(\frac{(n+2) t}{n}\right) \cosh (t)}, \quad v_{\mathrm{F}}=\frac{2(n+2)}{n}
$$


while the correction term $o\left(N^{-1-\epsilon}\right)$ contains an infinitesimally small positive $\varepsilon>0$ (for a more detailed description of the correction term see ref. [31]). The structure (5.21) looks typical for the low energy spectrum of a critical 1D system, where the states organize into the conformal towers [33]. However, an unusual feature is the presence of the $N$ dependent term $\propto b^{2}$ with $b=b(N)$. The latter turns out to be related to the eigenvalue of the quasi-shift operator computed on the state

$$
b(N)=\frac{n}{4 \pi} \log (B), \quad \mathbb{B} \Psi=B \Psi .
$$

It is important to keep in mind the following point. In writing the asymptotic formulae (5.21) as well as (5.23) we have implicitly assigned an $N$ dependence to a stationary state $\boldsymbol{\Psi}=\boldsymbol{\Psi}_{N}$. For a general lattice system there are obvious difficulties in doing this, i.e., forming Renormalization Group (RG) trajectories for individual states. Of course, since the space of states of a finite lattice model has different dimensions for different lattice sizes, the problem only makes sense for the low energy part of the spectrum. It is clear how to assign an $N$ dependence to the ground state or, for that matter, the lowest energy state in a disjoint sector of the space of states (say in a sector with given value of $S^{z}$ for the case under consideration). However forming individual RG flow trajectories for low energy stationary states that are densely distributed does not seem to be a trivial task. For the $\mathcal{Z}_{2}$ invariant spin chain the problem was discussed in ref. [31] and essentially exploits the integrable structure.

It turns out that the large $N$ behaviour of $b=b(N)$ for a state $\boldsymbol{\Psi}=\boldsymbol{\Psi}_{N}$ can be described through the asymptotic relation

$$
\epsilon^{-4 \mathrm{i} b} \mathrm{e}^{\frac{\mathrm{i}}{2} \delta_{\Psi}(b)}=\sigma+O\left(\log (1 / \epsilon)^{-\infty}\right), \quad \sigma=(-1)^{\frac{N}{2}-S^{z}} .
$$

Here $\delta_{\Psi}(b)$ depends on the stationary state under consideration and, for future convenience, we swap $N$ for the parameter $\epsilon$, defined as

$$
\epsilon^{-1}=\frac{2^{\frac{n+2}{n}} \Gamma\left(\frac{3}{2}+\frac{1}{n}\right)}{\sqrt{\pi} \Gamma\left(1+\frac{1}{n}\right)} N .
$$

Formula (5.24) resembles the quantization condition of a particle in a potential well of length $\propto \log (1 / \epsilon)$ with $\delta_{\boldsymbol{\Psi}}$ being the phase shift picked up by the particle at the turning points. It has the same form as the quantization condition (4.43) appearing in our discussion of the Lorentzian black hole NLSM. For the "primary" states, corresponding to vanishing levels $\mathrm{L}=\overline{\mathrm{L}}=0$ in eq. (5.21), the explicit formula for the phase shift was proposed in ref. [27]:

$$
\mathrm{e}^{\frac{\mathrm{i}}{2} \delta_{\Psi}(s)}=\frac{\Gamma\left(\frac{1}{2}+p-\mathrm{i} s\right) \Gamma\left(\frac{1}{2}+\bar{p}-\mathrm{i} s\right)}{\Gamma\left(\frac{1}{2}+p+\mathrm{i} s\right) \Gamma\left(\frac{1}{2}+\bar{p}+\mathrm{i} s\right)} \quad(\mathrm{L}=\overline{\mathrm{L}}=0)
$$

In the later work [34] a closed form expression for $\delta_{\boldsymbol{\Psi}}$ was obtained for an arbitrary low energy state.

There is a class of low energy states such that $\Im m(b(N)) \rightarrow 0$ as $N \rightarrow \infty$. In the scaling limit they form the space $\mathcal{H}^{(\text {cont })}$, whose linear structure is described through the 
decomposition (3.42) into the highest weight irreps of the $\bar{W}_{\infty} \otimes W_{\infty}$-algebra with $c=$ $2-\frac{6}{n+2}$. As usual, the exact knowledge of the phase shift is sufficient to derive the density of states that occurs in the continuous limit. In particular, let $\rho_{\bar{p}, p}^{(\overline{\mathrm{L}}, \mathrm{L})}(s) \Delta s$ be the number of states with given $p, \bar{p}, \mathrm{~L}$ and $\overline{\mathrm{L}}$ such that $\Re e(b(N)) \in(s, s+\Delta s)$. Then as $\epsilon \propto N^{-1} \rightarrow 0$, the density of states is given by

$$
\rho_{\bar{p}, p}^{(\overline{\mathrm{L}}, \mathrm{L})}(s)=\frac{2}{\pi} \operatorname{par}_{2}(\mathrm{~L}) \operatorname{par}_{2}(\overline{\mathrm{L}}) \log (1 / \epsilon)+\tilde{\rho}_{\bar{p}, p}^{(\overline{\mathrm{L}}, \mathrm{L})}(s)+o(1),
$$

where the finite part reads as

$$
\tilde{\rho}_{\bar{p}, p}^{(\overline{\mathrm{L}}, \mathrm{L})}(s)=\frac{1}{2 \pi \mathrm{i}} \partial_{s} \log \left[\left(\mathfrak{D}_{\bar{p}}^{(\overline{\mathrm{L}})}(s)\right)^{\operatorname{par}_{2}(\mathrm{~L})}\left(\mathfrak{D}_{p}^{(\mathrm{L})}(s)\right)^{\operatorname{par}_{2}(\overline{\mathrm{L}})}\right]
$$

with

$$
\mathfrak{D}_{\rho}^{(\ell)}(s)=\left(\frac{\Gamma\left(\frac{1}{2}+\rho-\mathrm{i} s\right)}{\Gamma\left(\frac{1}{2}+\rho+\mathrm{i} s\right)}\right)^{\operatorname{par}_{2}(\ell)} \prod_{a=0}^{\ell-1}\left[\frac{\left(\frac{1}{2}+a+\rho-\mathrm{i} s\right)\left(\frac{1}{2}+a-\rho-\mathrm{i} s\right)}{\left(\frac{1}{2}+a+\rho+\mathrm{i} s\right)\left(\frac{1}{2}+a-\rho+\mathrm{i} s\right)}\right]^{\operatorname{par}_{2}(\ell)-d_{a}(\ell)} .
$$

Recall that $\operatorname{par}_{2}(\ell)$ denotes the number of bipartitions of $\ell$. The integers $d_{a}(\ell)$ appearing in the exponent in the last formula are defined through their generating function,

$$
\chi_{a}(\mathrm{q}) \equiv \frac{1}{(\mathrm{q}, \mathrm{q})_{\infty}^{2}} \sum_{m=0}^{\infty}(-1)^{m} \mathrm{q}^{m a+\frac{m(m+1)}{2}}=\sum_{\ell=0}^{\infty} d_{a}(\ell) \mathrm{q}^{\ell} .
$$

Introduce the density matrix $\hat{\rho}$, which is an operator acting in $\mathcal{H}^{(\text {cont })}$ that commutes with the CFT Hamiltonian and total momentum operator

$$
\hat{H}_{\mathrm{CFT}}=L_{0}+\bar{L}_{0}-\frac{c}{12}, \quad \hat{P}_{\mathrm{CFT}}=L_{0}-\bar{L}_{0} .
$$

Being restricted to the level subspace of the highest weight representation $\overline{\mathcal{W}}_{\bar{p}, s} \otimes \mathcal{W}_{p, s}$, the operator $\hat{\rho}$ is given by

$$
\left.\hat{\rho}\right|_{\overline{\mathcal{W}}_{\bar{p}, s}^{(\overline{\mathrm{L}})} \otimes \mathcal{W}_{p, s}^{(\mathrm{L})}}=\left[\frac{2}{\pi} \log (1 / \epsilon)+\frac{\tilde{\rho}_{\bar{p}, p}^{(\overline{\mathrm{L}}, \mathrm{L})}(s)}{\operatorname{par}_{2}(\overline{\mathrm{L}}) \operatorname{par}_{2}(\mathrm{~L})}\right] \overline{\mathrm{q}}^{\Delta_{\bar{p}, s}-\frac{c}{24}+\overline{\mathrm{L}}} \mathrm{q}^{\Delta_{p, s}-\frac{c}{24}+\mathrm{L}} \hat{\mathbf{1}},
$$

where $\Delta_{p, s}$ is as in (3.40), while $\mathrm{q}$ and $\overline{\mathrm{q}}=\mathrm{q}^{*}$ are two complex conjugated numbers such that $|\mathrm{q}|<1$. The contribution of the low energy states forming $\mathcal{H}^{(\text {cont })}$ in the scaling limit to the spin chain partition function reads as

$$
\begin{aligned}
Z^{(\text {cont })=} & \sqrt{\frac{n}{\Im m(\tau)}} \frac{\log (1 / \epsilon)}{\pi(\overline{\mathrm{q}}, \overline{\mathrm{q}})_{\infty}^{2}(\mathrm{q}, \mathrm{q})_{\infty}^{2}} \sum_{\mathrm{u}, \mathrm{w}=-\infty}^{\infty} \overline{\mathrm{q}}^{-\frac{1}{12}+\frac{\bar{p}^{2}}{n+2}} \mathrm{q}^{-\frac{1}{12}+\frac{p^{2}}{n+2}} \\
& +\sum_{\mathrm{u}, \mathrm{w}=-\infty}^{\infty} \int_{-\infty}^{+\infty} \mathrm{d} s \sum_{\mathrm{L}, \overline{\mathrm{L}} \geq 0} \tilde{\rho}_{\bar{p}, p}^{(\overline{\mathrm{L}}, \mathrm{L})}(s) \overline{\mathrm{q}}^{-\frac{1}{12}+\frac{s^{2}}{n}+\frac{\bar{p}^{2}}{n+2}+\overline{\mathrm{L}}} \mathrm{q}^{-\frac{1}{12}+\frac{s^{2}}{n}+\frac{p^{2}}{n+2}+\mathrm{L}},
\end{aligned}
$$

where $\Im m(\tau)=-\frac{1}{4 \pi} \log (\mathrm{q} \overline{\mathrm{q}})$. This can be equivalently expressed as the trace of the density matrix:

$$
Z^{\text {(cont) }}=\operatorname{Tr}_{\mathcal{H}^{(\text {cont })}}(\hat{\rho})
$$


It is possible to perform the sum over $\mathrm{L}$ and $\overline{\mathrm{L}}$ in the second line of the formula (5.33) and show that

$$
\sum_{\mathrm{L}, \overline{\mathrm{L}} \geq 0} \tilde{\rho}_{\bar{p}, p}^{(\overline{\mathrm{L}}, \mathrm{L})}(s) \overline{\mathrm{q}}^{\overline{\mathrm{L}}} \mathrm{q}^{\mathrm{L}}=-\frac{r_{\bar{p}}(s, \overline{\mathrm{q}})+r_{p}(s, \mathrm{q})}{\pi(\overline{\mathrm{q}}, \overline{\mathrm{q}})_{\infty}^{2}(\mathrm{q}, \mathrm{q})_{\infty}^{2}}
$$

with

$$
\begin{aligned}
r_{p}(s, \mathrm{q})= & \frac{1}{2} \sum_{\sigma= \pm} \psi\left(\frac{1}{2}+p+\mathrm{i} \sigma s\right) \\
& +\oint_{|z|<1} \frac{\mathrm{d} z}{2 \pi \mathrm{i}} \frac{(\mathrm{q}, \mathrm{q})_{\infty}^{2}}{(z, \mathrm{q})_{\infty}\left(z^{-1} \mathrm{q}, \mathrm{q}\right)_{\infty}} \frac{1}{2} \sum_{\sigma, \sigma^{\prime}= \pm} \Phi\left(z, 1, \frac{1}{2}+\sigma^{\prime} p+\mathrm{i} \sigma s\right)
\end{aligned}
$$

Here $\psi(\alpha)=\partial_{\alpha} \log \Gamma(\alpha)$, while $\Phi(z, 1, \alpha)$ stands for the Lerch transcendent,

$$
\Phi(z, s, \alpha)=\sum_{m=0}^{\infty} \frac{z^{m}}{(m+\alpha)^{s}} .
$$

The relation (5.35) is useful for the numerical computation of $Z^{\text {(cont) }}$.

\section{Density matrix for the Lorentzian black hole NLSM}

Since $\mathcal{H}^{(\mathrm{LBH})}$ (4.14) is expected to be the space of states of the Lorentzian black hole NLSM, the equilibrium density matrix

$$
\hat{\rho}_{\mathrm{LBH}}: \quad \mathcal{H}^{(\mathrm{LBH})} \mapsto \mathcal{H}^{(\mathrm{LBH})}, \quad\left[\hat{\rho}_{\mathrm{LBH}}, \hat{H}_{\mathrm{CFT}}\right]=\left[\hat{\rho}_{\mathrm{LBH}}, \hat{P}_{\mathrm{CFT}}\right]=0
$$

is of special interest. The space $\mathcal{H}^{(\mathrm{LBH})}$ admits the decomposition

$$
\mathcal{H}^{(\mathrm{LBH})}=\bigoplus_{\mathrm{u}=0}^{\infty} \bigoplus_{\mathrm{w}=-\infty}^{\infty} \mathcal{H}_{\mathrm{u}, \mathrm{w}}^{(\mathrm{LBH})} .
$$

The operator $\hat{\rho}_{\mathrm{LBH}}$ acts invariantly in the sectors $\mathcal{H}_{\mathrm{u}, \mathrm{w}}^{(\mathrm{LBH})}$, which are the linear span of states with given quantum numbers $\mathrm{u}$ and $\mathrm{w}$. In a similar manner,

$$
\mathcal{H}^{(\text {cont })}=\bigoplus_{\mathrm{u}=-\infty}^{\infty} \bigoplus_{\mathrm{w}=-\infty}^{\infty} \mathcal{H}_{\mathrm{u}, \mathrm{w}}^{(\mathrm{cont})}
$$

and the sectors $\mathcal{H}_{\mathrm{u}, \mathrm{w}}^{(\text {cont })}$ are invariant subspaces for $\hat{\rho}$ (5.32) for any value of $\mathrm{k}$ including $\mathrm{k}=0$.

When $\mathrm{u}=0$ and for any $\mathrm{w} \in \mathbb{Z}$, the highest weight representations $\overline{\mathcal{W}}_{\bar{p}, s} \otimes \mathcal{W}_{p, s}$ with $\mathrm{k} \neq 0$ remain irreducible at $\mathrm{k}=0$. In the last case, the irreps are also $\mathcal{C}$ invariant. As a result, the subspace $\mathcal{H}_{0, \mathrm{w}}^{(\mathrm{LBH})}$ coincides with the $\mathcal{C}$ even component of $\mathcal{H}_{0, \mathrm{w}}^{(\mathrm{cont})}$. The latter occurs in the scaling limit of the low energy states with $S^{z}=0$ and given w. It turns out that for fixed $N \gg 1$ the difference between the number of $\mathcal{C}$ even and odd such low energy stationary states is of order one. Furthermore, in the limit $N \rightarrow \infty$ the density of $\mathcal{C}$ even and odd states with fixed L, $\overline{\mathrm{L}}, p=-\bar{p}=(n+2) \mathrm{w}$ is the same and coincides with $\frac{1}{2} \rho_{\bar{p}, p}^{(\overline{\mathrm{L}}, \mathrm{L})}$ described by eqs. (5.27)-(5.29) (for further details see section 17.4 from ref. [32]). Thus 
the restriction of the density matrix $\hat{\rho}_{\mathrm{LBH}}$ to the level subspaces of the irreps occurring in the decomposition of $\mathcal{H}_{0, \mathrm{w}}^{(\mathrm{LBH})}$ is given by

$$
\begin{aligned}
\left.\hat{\rho}_{\mathrm{LBH}}\right|_{\overline{\mathcal{W}}_{\bar{p}, s}^{(\overline{\mathrm{L}})} \otimes \mathcal{W}_{p, s}^{(\mathrm{L})}=} & \frac{1}{2}\left[\frac{2}{\pi} \log (1 / \epsilon)+\frac{\tilde{\rho}_{\bar{p}, p}^{(\overline{\mathrm{L}}, \mathrm{L})}(s)}{\operatorname{par}_{2}(\overline{\mathrm{L}}) \operatorname{par}_{2}(\mathrm{~L})}\right] \\
& \times \overline{\mathrm{q}}^{\Delta_{\bar{p}, s}-\frac{c}{24}+\overline{\mathrm{L}}} \mathrm{q}^{\Delta_{p, s}-\frac{c}{24}+\mathrm{L}} \hat{\mathbf{1}} \quad\left(p=-\bar{p}=\frac{1}{2}(n+2) \mathrm{w}, \mathrm{w} \in \mathbb{Z}\right) .
\end{aligned}
$$

For $\mathrm{w}=0$ the irreps $\overline{\mathcal{W}}_{\bar{p}, s} \otimes \mathcal{W}_{p, s}$, like in the previous case, remain irreducible at $\mathrm{k}=0$. Also the subspaces $\mathcal{H}_{\mathrm{u}, 0}^{(\mathrm{LBH})}$ and $\mathcal{H}_{\mathrm{u}, 0}^{(\mathrm{cont})}$ for $\mathrm{u}>0$ are equivalent. Hence the operator $\hat{\rho}_{\mathrm{LBH}}$ restricted to the level subspaces of the corresponding irreps is given by the same formula as (5.32):

$$
\begin{aligned}
\left.\hat{\rho}_{\mathrm{LBH}}\right|_{\mathcal{W}_{\bar{p}, s}^{(\overline{\mathrm{L}})} \otimes \mathcal{W}_{p, s}^{(\mathrm{L})}=} & {\left[\frac{2}{\pi} \log (1 / \epsilon)+\frac{\tilde{\rho}_{\bar{p}, p}^{(\overline{\mathrm{L}}, \mathrm{L})}(s)}{\operatorname{par}_{2}(\overline{\mathrm{L}}) \operatorname{par}_{2}(\mathrm{~L})}\right] } \\
& \times \overline{\mathrm{q}}^{\Delta_{\bar{p}, s}-\frac{c}{24}+\overline{\mathrm{L}}} \mathrm{q}^{\Delta_{p, s}-\frac{c}{24}+\mathrm{L}} \hat{\mathbf{1}} \quad\left(p=\bar{p}=\frac{1}{2}, 1, \frac{3}{2}, 2, \ldots\right) .
\end{aligned}
$$

However some care is needed in specializing the density of states to $p=\bar{p}=\frac{1}{2} \mathrm{u}$ for odd $\mathrm{u}$. In this case, as follows from eqs. (5.28) and (5.29) the function $\tilde{\rho}_{\bar{p}, p}^{(\overline{\mathrm{L}}, \mathrm{L})}(s)$ contains a simple pole at $s=0$, making its integration over $s$ ambiguous. The ambiguity can be resolved by starting with non-zero $\mathrm{k}$ and performing the limit $\mathrm{k} \rightarrow 0$. Using the Sokhotski-Plemelj formula one finds

$$
\begin{aligned}
\tilde{\rho}_{\bar{p}, p}^{(\overline{\mathrm{L}} \mathrm{L})}(s)= & \operatorname{P} \cdot \mathrm{V} \cdot\left(\tilde{\rho}_{\bar{p}, p}^{(\overline{\mathrm{L}}, \mathrm{L})}(s)\right) \\
& +\left(\operatorname{par}_{2}(\mathrm{~L}) d_{p-\frac{1}{2}}(\overline{\mathrm{L}})-\operatorname{par}_{2}(\overline{\mathrm{L}}) d_{p-\frac{1}{2}}(\mathrm{~L})\right) \delta(s) \quad\left(p=\bar{p}=\frac{1}{2}, \frac{3}{2}, \frac{5}{2}, \ldots\right)
\end{aligned}
$$

where the symbol P.V. stands for the principal value, while the integers $d_{a}(\mathrm{~L})$ are defined through their generating function in eq. (5.30).

When $\mathrm{u}>0$ and $\mathrm{w} \neq 0$ the subspaces $\mathcal{H}_{\mathrm{u}, \mathrm{w}}^{(\mathrm{LBH})}$ and $\mathcal{H}_{\mathrm{u}, \mathrm{w}}^{(\mathrm{cont})}$ do not coincide. In turn, the operator $\hat{\rho}_{\mathrm{LBH}}$ can not be obtained through a $\mathrm{k} \rightarrow 0$ limit of $\hat{\rho}$. Instead, one should return to the lattice system and compute the density of low energy stationary states that become part of $\mathcal{H}_{\mathrm{u}, \mathrm{w}}^{(\mathrm{LBH})}$ in the scaling limit. To express the result, together with the function $\mathfrak{D}_{\rho}^{(\ell)}(s)(5.29)$, we use

$$
\begin{aligned}
\widetilde{\mathfrak{D}}_{\rho}^{(\ell)}(s)= & \prod_{a=0}^{\ell-1}\left(\frac{\frac{1}{2}+a+\rho-\mathrm{i} s}{\frac{1}{2}+a+\rho+\mathrm{i} s}\right)^{\tilde{d}_{a}(\ell \mid+\rho)}\left(\frac{\frac{1}{2}+a-\rho-\mathrm{i} s}{\frac{1}{2}+a-\rho+\mathrm{i} s}\right)^{\tilde{d}_{a}(\ell \mid-\rho)} \\
& \times\left(\frac{\Gamma\left(\frac{1}{2}+\rho-\mathrm{i} s\right)}{\Gamma\left(\frac{1}{2}+\rho+\mathrm{i} s\right)}\right)^{\operatorname{par}_{2}(\ell)-\operatorname{par}_{2}(\ell-m r)}, \quad \text { where } \quad \rho=\frac{1}{2} r-\frac{1}{2}(n+2) m .
\end{aligned}
$$

The generating function for the exponents $\tilde{d}_{a}(\ell \mid \pm \rho)$ entering into the above product is given by

$$
\sum_{\ell=0}^{\infty} \tilde{d}_{a}(\ell \mid \pm \rho) \mathrm{q}^{\ell}=\frac{1}{(\mathrm{q}, \mathrm{q})_{\infty}^{2}} \sum_{j=1}^{\infty}(-1)^{j+1} \mathrm{q}^{j a+\frac{j(j+1)}{2}}\left(1-\mathrm{q}^{(m \pm j) r}\right)
$$


Note that we take by definition $\operatorname{par}_{2}(\ell-m r)=0$ when $\ell<m r$. With this notation, the density matrix restricted to the subspaces $\overline{\mathcal{W}}_{\bar{p}, s}^{(\overline{\mathrm{L}})} \otimes \mathcal{W}_{p, s}^{(\mathrm{L})}$ with

$$
p=\frac{1}{2} \mathrm{u}+\frac{1}{2}(n+2) \mathrm{w}, \quad \bar{p}=\frac{1}{2} \mathrm{u}-\frac{1}{2}(n+2) \mathrm{w} \quad \text { and } \quad \mathrm{u}>0, \mathrm{w} \neq 0
$$

is given by

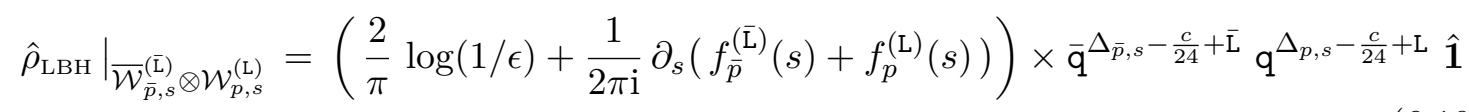

where

$$
f_{\rho}^{(\ell)}(s)=\left\{\begin{array}{ll}
\frac{\log \mathfrak{D}_{\rho}^{(\ell)}(s)}{\operatorname{par}_{2}(\ell)} & \text { for } m<0 \\
\frac{\log \widetilde{\mathfrak{D}}_{\rho}^{(\ell)}(s)}{\operatorname{par}_{2}(\ell)-\operatorname{par}_{2}(\ell-m r)} & \text { for } m>0
\end{array} \quad\left(\rho=\frac{1}{2} r-\frac{1}{2}(n+2) m\right) .\right.
$$

\section{Low energy states of the $\mathcal{Z}_{2}$ invariant spin chain in the scaling limit. Discrete spectrum}

The low energy spectrum of the $\mathcal{Z}_{2}$ invariant spin chain consists of two classes of states which are distinguished by the large $N$ behaviour of the eigenvalue of the quasi-shift operator. Up till now we have been focused on the states, where the imaginary part of $b(N)$ (5.23) vanishes as $N \rightarrow \infty$. In the scaling limit these organize into the space $\mathcal{H}^{(\text {cont) }}$. For $(n+2) \mathrm{k} \notin \mathbb{Z}$ the linear structure of this space is given by (3.42), while its Hermitian structure is specified through the conjugation conditions (3.51) along with the inner product of the $\bar{W}_{\infty} \otimes W_{\infty}$ primary states (3.52), (5.13). For the states from the second class $\lim _{N \rightarrow \infty} b(N)$ is a pure imaginary number whose admissible values form a discrete set. The energy-momentum spectrum is still described by the large $N$ asymptotic formula (5.21). We'll refer to the space into which the states organize in the scaling limit as $\mathcal{H}^{\text {(disc) }}$. Here we quote the results of ref. [32] regarding its linear and Hermitian structure for $(n+2) \mathrm{k} \notin \mathbb{Z}$.

\subsection{Decomposition into the irreps of the algebra of extended conformal sym- metry}

The space $\mathcal{H}^{(\text {disc) }}$ is splitted into two sectors

$$
\mathcal{H}^{(\mathrm{disc})}=\mathcal{H}^{(\mathrm{disc},+)} \bigoplus \mathcal{H}^{(\mathrm{disc},-)} .
$$

In turn for each of the spaces,

$$
\mathcal{H}^{(\mathrm{disc}, \pm)}=\bigoplus_{\mathrm{u}, \mathrm{w} \in \mathbb{Z}} \mathcal{H}_{\mathrm{u}, \mathrm{w}}^{(\mathrm{disc}, \pm)}
$$

where, similar to (6.3), the components $\mathcal{H}_{\mathrm{u}, \mathrm{w}}^{(\mathrm{disc}, \pm)}$ are formed by the linear span of states with fixed value of $S^{z}=\mathrm{u}$ and winding number w. For $\mathcal{H}_{\mathrm{u}, \mathrm{w}}^{\text {(disc, }+ \text { ) }}$ the linear decomposition 
into irreps of the $\bar{W}_{\infty} \otimes W_{\infty}$ - algebra is given by

$$
\mathcal{H}_{\mathrm{u}, \mathrm{w}}^{(\mathrm{disc},+)}=\bigoplus_{\sigma= \pm 1}\left(\mathcal{H}_{\mathrm{u}, \mathrm{w}, \sigma}^{(1,+)} \oplus \mathcal{H}_{\mathrm{u}, \mathrm{w}, \sigma}^{(2,+)}\right)
$$

with

$$
\mathcal{H}_{\mathrm{u}, \mathrm{w}, \sigma}^{(1,+)}=\bigoplus_{a \in \Sigma(p)} \overline{\mathcal{W}}_{\bar{p}, \sigma \mathrm{i} q_{a}} \otimes \mathcal{W}_{p, \sigma \mathrm{i} \mathfrak{q}_{a}}, \quad \mathcal{H}_{\mathbf{u}, \mathrm{w}, \sigma}^{(2,+)}=\bigoplus_{a \in \Sigma(\bar{p})} \overline{\mathcal{W}}_{\bar{p}, \sigma \mathrm{i} \bar{q}_{a}} \otimes \mathcal{W}_{p, \sigma \mathrm{i} \bar{q}_{a}}
$$

Here $\mathfrak{q}_{a}$ and $\overline{\mathfrak{q}}_{a}$ are defined as

$$
\mathfrak{q}_{a}=-p-\frac{1}{2}-a, \quad \overline{\mathfrak{q}}_{a}=-\bar{p}-\frac{1}{2}-a .
$$

The summation in (7.4) is taken over the non-negative integer $a$ restricted to the set

$$
\Sigma(p)=\left\{a: a \in \mathbb{Z}_{+},-p-\frac{n+2}{4} \leq a<-\frac{1}{2}-p\right\}
$$

as well as $\Sigma(\bar{p})$, which is given by the same formula with $p$ substituted by $\bar{p}$.

The linear structure of $\mathcal{H}_{\mathrm{u}, \mathrm{w}}^{(\text {disc,-) }}$ is more involved. To describe it, in addition to $p, \bar{p}$, $\mathfrak{q}_{a}$ and $\overline{\mathfrak{q}}_{a}$, we use the notation

$$
\begin{aligned}
p_{+} & =\frac{1}{2} \mathrm{u}+\frac{1}{2}(n+2)(\mathrm{k}+\mathrm{w}+1), & \bar{p}_{+} & =\frac{1}{2} \mathrm{u}-\frac{1}{2}(n+2)(\mathrm{k}+\mathrm{w}+1) \\
p_{-} & =\frac{1}{2} \mathrm{u}+\frac{1}{2}(n+2)(\mathrm{k}+\mathrm{w}-1), & \bar{p}_{-} & =\frac{1}{2} \mathrm{u}-\frac{1}{2}(n+2)(\mathrm{k}+\mathrm{w}-1) \\
\mathfrak{q}_{a}^{\prime} & =-p-\frac{n+1}{2}-a, & \overline{\mathfrak{q}}_{a}^{\prime} & =-\bar{p}-\frac{n+1}{2}-a .
\end{aligned}
$$

Then

$$
\mathcal{H}_{\mathrm{u}, \mathrm{w}}^{(\mathrm{disc},-)}=\bigoplus_{\sigma= \pm 1}\left(\mathcal{H}_{\mathrm{u}, \mathrm{w}, \sigma}^{(1,-)} \oplus \mathcal{H}_{\mathrm{u}, \mathrm{w}, \sigma}^{(2,-)} \oplus \mathcal{H}_{\mathrm{u}, \mathrm{w}, \sigma}^{(3,-)} \oplus \mathcal{H}_{\mathrm{u}, \mathrm{w}, \sigma}^{(4,-)}\right)
$$

and the decomposition of each of the four spaces $\mathcal{H}_{\mathrm{u}, \mathrm{w}, \sigma}^{(i,-)}$ into the irreps of the $\bar{W}_{\infty} \otimes W_{\infty}$ algebra reads explicitly as

$$
\begin{array}{ll}
\mathcal{H}_{\mathrm{u}, \mathrm{w}, \sigma}^{(1,-)}=\bigoplus_{a \in \Sigma_{1}(p)} \overline{\mathcal{W}}_{\bar{p}_{+}, \sigma \mathrm{i} \mathfrak{q}_{a}^{\prime}} \otimes \mathcal{W}_{p_{+}, \sigma \mathrm{iq} q_{a}^{\prime}}, & \mathcal{H}_{\mathrm{u}, \mathrm{w}, \sigma}^{(2,-)}=\bigoplus_{a \in \Sigma_{2}(p)} \overline{\mathcal{W}}_{\bar{p}, \sigma \mathrm{i} \mathfrak{q}_{a}} \otimes \mathcal{W}_{p_{+}, \sigma \mathrm{iq} q_{a}^{\prime}} \\
\mathcal{H}_{\mathrm{u}, \mathrm{w}, \sigma}^{(3,-)}=\bigoplus_{a \in \Sigma_{2}(\bar{p})} \overline{\mathcal{W}}_{\bar{p}_{-}, \sigma \mathrm{i} \mathrm{q}_{a}^{\prime}} \otimes \mathcal{W}_{p, \sigma \mathrm{i} \overline{\mathfrak{q}}_{a}}, & \mathcal{H}_{\mathrm{u}, \mathrm{w}, \sigma}^{(4,-)}=\bigoplus_{a \in \Sigma_{1}(\bar{p})} \overline{\mathcal{W}}_{\bar{p}_{-,}, \sigma \mathrm{i} \overline{\mathfrak{q}}_{a}^{\prime}} \otimes \mathcal{W}_{p_{-}, \sigma \mathrm{i} \bar{q}_{a}^{\prime}}
\end{array}
$$

The summation index $a$ takes negative integer values and runs over the sets

$$
\begin{aligned}
& \Sigma_{1}(p)=\left\{a: a \in \mathbb{Z}_{-},-p-\frac{n+2}{4} \leq a<-\frac{1}{2}-p \& a<-\mathrm{u}\right\} \\
& \Sigma_{2}(p)=\left\{a: a \in \mathbb{Z}_{-},-p-\frac{n+2}{4} \leq a<-\frac{1}{2}-p \& a \geq-\mathrm{u}\right\}
\end{aligned}
$$

and $\Sigma_{1}(\bar{p}), \Sigma_{2}(\bar{p})$, which are defined by the analogous formulae. 
All the chiral irreps appearing in the decomposition of $\mathcal{H}^{(\mathrm{disc})}$ are of the form

$$
\mathcal{W}_{\rho, \nu}: \quad \Im m(\rho)=\Re e(\nu)=0, \quad \rho+\frac{1}{2} \pm \mathrm{i} \nu \in \mathbb{Z}
$$

with some choice of the sign \pm . In this case the irrep of the $W_{\infty}$-algebra does not coincide with the Verma module as the latter contains null vector(s). It turns out that if either $\rho+\frac{1}{2}+\mathrm{i} \nu=-a \in \mathbb{Z}$ or $\rho+\frac{1}{2}-\mathrm{i} \nu=-a \in \mathbb{Z}$ and $2 \rho \notin \mathbb{Z}$, the character of $\mathcal{W}_{\rho, \nu}$ is given by [22]

$$
\operatorname{ch}_{\rho, \nu}(\mathrm{q})=\frac{\mathrm{q}^{-\frac{1}{12}+\frac{\nu^{2}}{n}+\frac{\rho^{2}}{n+2}}}{(\mathrm{q}, \mathrm{q})_{\infty}^{2}} \sum_{m=0}^{\infty}(-1)^{m} \mathrm{q}^{m\left|a+\frac{1}{2}\right|+\frac{m^{2}}{2}} \quad \begin{aligned}
& \rho+\frac{1}{2} \pm \mathrm{i} \nu \in \mathbb{Z} \\
& \rho \text { generic } .
\end{aligned}
$$

Note that when $2 \rho, 2 \mathrm{i} \nu \in \mathbb{Z}$, while $2(\rho+\mathrm{i} \nu)$ is an odd integer then, assuming also that $n$ is irrational, ${ }^{9}$

$$
\operatorname{ch}_{\rho, \nu}(\mathrm{q})=\frac{\mathrm{q}^{-\frac{1}{12}+\frac{\nu^{2}}{n}+\frac{\rho^{2}}{n+2}}}{(\mathrm{q}, \mathrm{q})_{\infty}^{2}} \sum_{m=0}^{\infty}(-1)^{m} \mathrm{q}^{\frac{m^{2}}{2}}\left(\mathrm{q}^{m|| \rho|-| \nu||}-\mathrm{q}^{(m+1)(|\rho|+|\nu|+1)-\frac{1}{2}}\right)
$$

where $\Im m(\rho)=\Re e(\nu)=0$ such that $|\rho| \pm|\nu| \in \frac{1}{2}+\mathbb{Z}$.

The linear decomposition of $\mathcal{H}^{(\text {disc })}$ described above together with the formula (7.12) for the character are sufficient to compute the partition function

$$
Z^{(\text {disc })}=\operatorname{Tr}_{\mathcal{H}^{(\text {disc })}}\left[\overline{\mathrm{q}}^{\bar{L}_{0}-\frac{c}{24}} \mathrm{q}^{L_{0}-\frac{c}{24}}\right]
$$

To write the result in a compact way we borrow the notation $\chi_{(\mathbf{j}, a-\mathfrak{j})}^{d}(\mathbf{q})$ from ref. [11]. Up to a simple factor, this function coincides with $\chi_{a}(\mathrm{q})$ defined in eq. (5.30),

$$
\chi_{(\mathbf{j}, a-\mathrm{j})}^{d}(\mathrm{q}) \equiv \mathrm{q}^{-\frac{1}{12}-\frac{\left(\mathrm{j}+\frac{1}{2}\right)^{2}}{n}+\frac{(\mathrm{j}-a)^{2}}{n+2}} \chi_{a}(\mathrm{q}) \quad(a \in \mathbb{Z})
$$

It is related to the character (7.12) as

$$
\chi_{(\mathfrak{j}, a-\mathfrak{j})}^{d}(\mathrm{q})=\operatorname{ch}_{a-\mathfrak{j}, \mathrm{i}\left(\mathrm{j}+\frac{1}{2}\right)}(\mathrm{q}) \times\left\{\begin{array}{lll}
1 & \text { for } & a \geq 0 \\
\mathrm{q}^{-a} & \text { for } & a<0
\end{array} .\right.
$$

Also introduce the notation $\mathfrak{J}(\mathrm{v}, \mathrm{u})$ for the finite set of all real numbers belonging to the half-open segment $\left[-\frac{n+1}{2},-\frac{1}{2}\right)$ such that

$$
\mathfrak{J}(\mathrm{v}, \mathrm{u}) \equiv\left\{\mathfrak{j}: \mathfrak{j} \in\left[-\frac{n+1}{2},-\frac{1}{2}\right) \& \mathfrak{j}-\frac{1}{2} \mathrm{v}-\frac{1}{2}(n+2)(\mathrm{k}+\mathrm{u}) \in \mathbb{Z}\right\}
$$

\footnotetext{
${ }^{9}$ For integer $n=2,3, \ldots$ the corresponding formula for the character was first obtained in ref. [35] (see also [22]). In addition note that the formulae (3.44), (4.16), (7.12), (7.13) for the characters, which assume that $c=2-\frac{6}{n+2}<2$, can be applied to the case $c>2$ if one makes the formal substitutions $n \rightarrow-n-2$, $\rho \rightarrow \mathrm{i} s, \nu \rightarrow \mathrm{i} p$. The central charge and highest weight of the irrep would be parameterized as in (8.3) and (8.4) below (see refs. $[17,36])$.
} 
Then the calculation of the trace over the space $\mathcal{H}^{(\mathrm{disc})}=\mathcal{H}^{(\mathrm{disc},+)} \oplus \mathcal{H}^{(\mathrm{disc},-)}$ yields ${ }^{10}$

$$
Z^{(\mathrm{disc})}=2 \sum_{\mathrm{v}, \mathrm{u} \in \mathbb{Z}} \sum_{\mathbf{j} \in \mathfrak{J}(\mathrm{v}, \mathrm{u})} \chi_{(\mathrm{j}, \overline{\mathrm{p}})}^{d}(\overline{\mathrm{q}}) \chi_{(\mathrm{j},-\mathrm{p})}^{d}(\mathrm{q})
$$

where

$$
\overline{\mathrm{p}}=\frac{1}{2} \mathrm{v}-\frac{1}{2}(n+2)(\mathrm{k}+\mathrm{u}), \quad \mathrm{p}=\frac{1}{2} \mathrm{v}+\frac{1}{2}(n+2)(\mathrm{k}+\mathrm{u}) .
$$

The overall factor of 2 in the formula for $Z^{(\text {disc) }}$ occurs due to the global $\mathcal{Z}_{2}$ invariance of the model.

The following comment is in order here. For arbitrary values of $k$, the inclusion of the endpoints into the interval for $\mathfrak{j}$ in $(7.17)$ has no effect on the set $\mathfrak{J}(\mathrm{v}, \mathrm{u})$. However for $\mathrm{k}=0$ and with $n$ generic, $\mathfrak{j}$ may coincide with $-\frac{n+1}{2}$ or $-\frac{1}{2}$. Taking the limit $\mathrm{k} \rightarrow 0$ of $Z^{\text {(disc) }}$ one finds that in order for (7.18) to correctly describe the contribution of the discrete spectrum to the partition function $Z^{\text {(disc) }}$ with $\mathrm{k}=0$ one of the endpoints in (7.17) must be included. The choice of whether to include $\mathfrak{j}=-\frac{n+1}{2}$ or $\mathfrak{j}=-\frac{1}{2}$ does not matter, since they correspond to the contribution of the same states to $Z^{\text {(disc) }}$.

\subsection{Hermitian structure}

The space $\mathcal{H}^{(\text {cont })}$ is built out of the $\bar{W}_{\infty} \otimes W_{\infty}$ irreps whose highest weights, $\left(\bar{\omega}_{2}, \bar{\omega}_{3} ; \omega_{2}, \omega_{3}\right)$, are real. In this case the inner product was introduced via the conjugation conditions (3.51) for the $W$ currents. On the other hand, for the irreps appearing in the decomposition of $\mathcal{H}^{\text {(disc) }}$, though $\omega_{2}, \bar{\omega}_{2}$ are real, $\omega_{3}$ and $\bar{\omega}_{3}$ are pure imaginary. As was discussed in the work [32], the natural inner product for such irreps is the one that is induced by the conjugation conditions

$$
\left[W_{j}(u)\right]^{*}=(-1)^{j} W_{j}\left(u^{*}\right), \quad\left[\bar{W}_{j}(\bar{u})\right]^{*}=(-1)^{j} \bar{W}_{j}\left(\bar{u}^{*}\right) .
$$

It turns out that the latter occur in the scaling limit of the finite length spin chain with the matrix conjugation being defined as

$$
\hat{\mathrm{O}}^{*}=\hat{\mathrm{X}}_{*}^{-1} \hat{\mathrm{O}}^{\dagger} \hat{\mathrm{X}}_{*}, \quad \hat{\mathrm{O}} \in \operatorname{End}\left(\mathscr{V}_{N}\right) .
$$

Here $\hat{X}_{*}=\hat{X}_{*}^{\dagger}$ is related to the matrix $\hat{X}_{\star}$ appearing in eq. (5.11) via the generator of the $\mathcal{Z}_{2}$ symmetry:

$$
\hat{\mathrm{X}}_{*}=\hat{\mathrm{X}}_{\star} \hat{\mathcal{D}}
$$

Since the Hamiltonian commutes with $\hat{\mathcal{D}}$, it follows from (5.12) that it is Hermitian w.r.t. the $:$-conjugation as well:

$$
\mathbb{H}^{*}=\mathbb{H}^{\star}=\mathbb{H}
$$

Note that for the lattice translation operator (5.20),

$$
\mathbb{K}^{*}=\mathbb{K}^{\star}=\mathbb{K}^{-1}
$$

\footnotetext{
${ }^{10}$ In the formula $(7.18)$ for $Z^{(\text {disc })}$ the integers $\mathrm{v}$ and $\mathrm{u}$ are formal summation variables, which can not be identified with the eigenvalue of $\mathbb{S}^{z}$ and the winding number w. In turn the notation $\mathrm{p}$ and $\overline{\mathrm{p}}$ in $(7.19)$ should not be confused with $p$ and $\bar{p}$ from (3.18).
} 
Let

$$
\Psi_{\bar{\rho}, \rho, \bar{\nu}, \nu} \in \mathcal{W}_{\bar{\rho}, \bar{\nu}} \otimes \mathcal{W}_{\rho, \nu} \subset \mathcal{H}^{(\mathrm{disc})}
$$

be the $\bar{W}_{\infty} \otimes W_{\infty}$ primary state, where the admissible set of values for $(\bar{\rho}, \rho, \bar{\nu}, \nu)$ is described by the formulae (7.1)-(7.10). The inner product of these states, together with the conjugation conditions (7.20) for the $W$ currents, fully specify the structure of the pseudoHilbert space for $\mathcal{H}^{\text {(disc) }}$. The results of [32] imply that the normalization of the primary $\bar{W}_{\infty} \otimes W_{\infty}$ states can be chosen in such a way that

$$
\left\langle\Psi_{\bar{\rho}, \rho^{\prime}, \bar{\nu}^{\prime}, \nu^{\prime}}, \Psi_{\bar{\rho}, \rho, \bar{\nu}, \nu}\right\rangle_{\mathrm{disc}}=\sigma f_{\bar{\rho}, \bar{\nu}} f_{\rho, \nu} \delta_{\bar{\rho}^{\prime}, \bar{\rho}} \delta_{\rho^{\prime}, \rho} \delta_{\bar{\nu}^{\prime}, \bar{\nu}} \delta_{\nu^{\prime}, \nu},
$$

where

$$
f_{\rho, \nu}=\frac{\Gamma(1+2 \rho) \Gamma\left(\frac{1}{2}+\rho-|\nu|\right)}{2 \pi \Gamma\left(1+\frac{2 \rho}{n+2}\right)} \times \begin{cases}(-1)^{a} a ! & \text { if } \frac{1}{2}+\rho+|\nu|=-a=0,-1, \ldots \\ \frac{2 \pi}{\Gamma\left(\frac{1}{2}+\rho+|\nu|\right)} & \text { otherwise }\end{cases}
$$

Here the sign factor $\sigma=(-1)^{N / 2-S^{z}}$ is the same one that enters into the quantization condition (5.24) and depends on whether, in constructing the RG trajectories, $N / 2-S^{z}$ is kept to be an even or an odd number. Let's reiterate that the above formulae are not literally applicable when $(n+2) \mathrm{k} \in \mathbb{Z}$, including the case $\mathrm{k}=0$.

The fact that the Hermitian structures for the spaces $\mathcal{H}^{(\mathrm{cont})}$ and $\mathcal{H}^{\text {(disc) }}$ correspond to different conjugation conditions in the algebra of extended conformal symmetry suggests that the states from these two spaces can not be interpreted simultaneously as normalizable states within a single conformal field theory. In this paper we argue that for generic values of $k, \mathcal{H}^{\text {(cont) }}$ serves as the pseudo-Hilbert space for the gauged $\operatorname{SL}(2, \mathbb{R})$ WZW model. Perhaps the simplest idea for the field theory whose quantization results in the pseudo-Hilbert space $\mathcal{H}^{\text {(disc) }}$, is the model described by the same Lagrangian density and constraints (2.13), (2.14) as well as the boundary conditions (2.19) for the WZW currents and (2.35) for $\partial_{\mu} \eta$. However the fields now are subject to different reality conditions. The classical counterpart to $(7.20)$ reads as

$$
\left(W_{j}^{(c l)}\right)^{*}=(-1)^{j} W_{j}^{(c l)}, \quad\left(\bar{W}_{j}^{(c l)}\right)^{*}=(-1)^{j} \bar{W}_{j}^{(c l)} .
$$

In view of eq. (2.50) this would follow from the reality conditions

$$
\left(L^{3}\right)^{*}=-L^{3}, \quad\left(L^{ \pm}\right)^{*}=L^{\mp}, \quad\left(R^{3}\right)^{*}=-R^{3}, \quad\left(R^{ \pm}\right)^{*}=R^{\mp}
$$

imposed on the classical WZW currents and

$$
(\partial \eta)^{*}=-\partial \eta, \quad(\bar{\partial} \eta)^{*}=-\bar{\partial} \eta
$$

for the Gaussian field. Furthermore i $\eta$ is expected to be a real and compactified field,

$$
\mathrm{i} \eta \sim \mathrm{i} \eta+2 \pi .
$$

The latter implies that the zero mode momenta $P_{\eta}$ and $\bar{P}_{\eta}(2.39)$ are no longer equal, but instead

$$
\mathrm{i}\left(P_{\eta}-\bar{P}_{\eta}\right) \in \mathbb{Z}
$$


Notice that $B=\mathrm{e}^{2 \pi P_{\eta}}$ and $\bar{B}=\mathrm{e}^{2 \pi \bar{P}_{\eta}}$ appearing in the boundary conditions (2.46) still coincide. Such reality and boundary conditions for the currents correspond to the $\mathrm{SU}(2)$ WZW model gauged over the compact subgroup. However, they are not enough to fully specify the field theory. In the $\operatorname{SL}(2, \mathbb{R})$ case there were the additional constraints $(2.32)$ and (2.34) whose motivation relied on the fact that the WZW field $\mathbf{G} \in \mathrm{SL}(2, \mathbb{R})$. At the moment, it is not clear to us what extra conditions need to imposed for the $\mathrm{SU}(2)$ case.

\section{Density matrix for the Euclidean black hole NLSM}

In the work [27] the authors put forward the pioneering conjecture that the Euclidean black hole NLSM is the CFT governing the scaling limit of the $\mathcal{Z}_{2}$ invariant spin chain in the domain of the anisotropy parameter $\gamma \in\left(0, \frac{\pi}{2}\right)$. This is not quite in line with what is proposed here. Nevertheless the study of the spin chain yields a certain density matrix whose trace coincides with the modular invariant partition function $Z_{\mathrm{EBH}}$. This makes it a good candidate for the equilibrium density matrix $\hat{\rho}_{\mathrm{EBH}}$.

The classical field theory is described by the action (1.3b), where $U$ and $U^{*}$ are a pair of complex conjugated fields. Usually they are assumed to be periodic, however, it is useful to consider the more general quasiperiodic boundary conditions

$$
U(t, x+2 \pi)=\mathrm{e}^{2 \pi \mathrm{ik}} U(t, x)
$$

Thus defined, the model possesses a U(1) symmetry with the corresponding Noether current given by

$$
I_{\mu}=\frac{1}{2 \mathrm{i}} \frac{U^{*} \partial_{\mu} U-U^{*} \partial_{\mu} U}{1+U U^{*}} .
$$

There is also an infinite set of chiral currents, which form the classical $\bar{W}_{\infty} \otimes W_{\infty}$ Poisson algebra. The quantization of the latter leads to the algebra of extended conformal symmetry with central charge $c>2$. The Hilbert space of the quantum theory can be classified according to the highest weight irreps of the $\bar{W}_{\infty} \otimes W_{\infty}$ - algebra. It is convenient to parameterize the central charge and the highest weight of the irreps, $\boldsymbol{\omega}=\left(\omega_{2}, \omega_{3}\right)$, using $n, s$ and $p$ as

$$
c=2+\frac{6}{n}>2
$$

and

$$
\begin{aligned}
& \omega_{2}=\frac{s^{2}+\frac{1}{4}}{n}+\frac{p^{2}}{n+2} \equiv \Delta_{p, s}^{(c>2)} \\
& \omega_{3}=\frac{2 p}{\sqrt{n+2}}\left(\frac{s^{2}}{n}+\frac{(2+3 n) p^{2}}{3 n(n+2)}-\frac{2 n+1}{12 n}\right) .
\end{aligned}
$$

To avoid confusion let us emphasize that in these relations $n>0, s$ and $p$ are formal parameters, without the meaning that was assigned to them in the previous sections. The Hilbert space of the NLSM contains both a continuous $\mathcal{H}_{\mathrm{EBH}}^{(\text {cont })}$ and a discrete component $\mathcal{H}_{\mathrm{EBH}}^{(\text {disc })}$. The linear decomposition of the continuous one into the irreps of the $\bar{W}_{\infty} \otimes W_{\infty}$-algebra 
is given by $[6-12]$

$$
\mathcal{H}_{\mathrm{EBH}}^{(\mathrm{cont})}=\bigoplus_{\mathrm{v}, \mathrm{w}=-\infty}^{+\infty} \int_{s>0}^{\oplus} \mathrm{d} s \overline{\mathcal{W}}_{\bar{p}, s}^{(c>2)} \otimes \mathcal{W}_{p, s}^{(c>2)}, \quad \text { where } \begin{aligned}
p & =\frac{1}{2} \mathrm{v}+\frac{1}{2}(n+2)(\mathrm{k}+\mathrm{w}) \\
\bar{p} & =\frac{1}{2} \mathrm{v}-\frac{1}{2}(n+2)(\mathrm{k}+\mathrm{w}) .
\end{aligned}
$$

Here $\mathrm{v}$ is the eigenvalue of the $\mathrm{U}(1)$ conserved charge $\hbar^{-1} \oint \mathrm{d} x I_{0}$ associated with the Noether current (8.2). It takes integer values provided that the Planck constant is identified with $n$ as

$$
\hbar=\frac{2 \pi}{n+2} .
$$

The integer w may be interpreted as a winding number related to the fact that the boundary condition (8.1) is invariant w.r.t. the substitution $\mathrm{k} \mapsto \mathrm{k}+\mathrm{w}$ with $\mathrm{w} \in \mathbb{Z}$. Let us note that the highest weight (8.4) is not sensitive to the sign of $s$. Due to this the direct integral in (8.5) is restricted to positive values of $s$. For the states at the level $\overline{\mathrm{L}}$ and $\mathrm{L}$ in the irrep $\overline{\mathcal{W}}_{\bar{p}, s}^{(c>2)} \otimes \mathcal{W}_{p, s}^{(c>2)}$, the corresponding energy $E=\Delta+\bar{\Delta}-\frac{c}{12}$ in terms of the parameters $n$, $s$ and $p$ reads as

$$
E=-\frac{1}{6}+\frac{2 s^{2}}{n}+\frac{p^{2}+\bar{p}^{2}}{n+2}+\mathrm{L}+\overline{\mathrm{L}} .
$$

It is worth mentioning that the space of states $\mathcal{H}_{\mathrm{EBH}}=\mathcal{H}_{\mathrm{EBH}}^{(\mathrm{cont})} \oplus \mathcal{H}_{\mathrm{EBH}}^{(\text {disc })}$ is equipped with a positive definite inner product [5] such that the Fourier modes of the $W$ and $\bar{W}$ currents, generating the $\bar{W}_{\infty} \otimes W_{\infty}$-algebra with $c>2$, satisfy the conjugation conditions

$$
\left[\widetilde{W}_{j}(m)\right]^{\dagger}=\widetilde{W}_{j}(-m), \quad\left[\widetilde{W}_{j}(m)\right]^{\dagger}=\widetilde{W}_{j}(-m) .
$$

In the works $[9,10]$ a modular invariant partition function was found for the Euclidean black hole NLSM with periodic boundary conditions imposed on the fundamental field $(\mathrm{k}=0)$. It is straightforward to generalize the result to the case of twisted boundary conditions. This yields ${ }^{11}$

$$
Z_{\mathrm{EBH}}=\frac{\sqrt{n(n+2)}}{\Im m(\tau)} \sum_{\mathrm{a}, \mathrm{b} \in \mathbb{Z}} \int_{D_{\epsilon}} \mathrm{d}^{2} z \mathrm{e}^{-\frac{\pi(n+2)}{\Im m(\tau)}|z+\mathrm{a}+(\mathrm{b}+\mathrm{k}) \tau|^{2}+\frac{2 \pi}{\Im m(\tau)}(\Im m(z))^{2}}\left|\frac{\eta(\tau)}{\vartheta_{1}(z \mid \tau)}\right|^{2},
$$

where $\vartheta_{1}$ and $\eta$ are the standard elliptic theta and Dedekind eta functions:

$$
\begin{aligned}
\vartheta_{1}(u \mid \tau) & =2 \mathrm{q}^{\frac{1}{8}} \sin (\pi u)\left(\mathrm{e}^{2 \pi \mathrm{i} u} \mathrm{q}, \mathrm{q}\right)_{\infty}\left(\mathrm{e}^{-2 \pi \mathrm{i} u} \mathrm{q}, \mathrm{q}\right)_{\infty}(\mathrm{q}, \mathrm{q})_{\infty} & \\
\eta(\tau) & =\mathrm{q}^{\frac{1}{24}}(\mathrm{q}, \mathrm{q})_{\infty} & \left(\mathrm{q}=\mathrm{e}^{2 \pi \mathrm{i} \tau}\right) .
\end{aligned}
$$

Note that the dependence on the twist parameter $\mathrm{k}$ manifests itself only as a shift of the summation variable $\mathrm{b} \mapsto \mathrm{b}+\mathrm{k}$, which appears in the exponent in the integrand in (8.9). The integral is taken over the parallelogram $D$ in the complex $z$ plane with vertices at

\footnotetext{
${ }^{11}$ The formulae for the partition function presented in the works $[9,10]$ is twice $Z_{\mathrm{EBH}}$ given by (8.9) with $\mathrm{k}=0$. This is related to the fact that the corresponding NLSM was obtained by gauging the U(1) symmetry, $\mathbf{g} \mapsto \mathbf{h} \mathbf{g} \mathbf{h}\left(\mathbf{h}=\mathrm{e}^{\mathrm{i} \frac{\mathrm{i} \phi}{2} \sigma^{y}}\right)$, of the $\mathrm{SL}(2, \mathbb{R})$ WZW model. This results in two copies of the Euclidean black hole NLSM. A similar occurrence happens for the Lorentzian black hole NLSM, as mentioned in section 2.2.
} 
$z= \pm \frac{1}{2} \pm \frac{1}{2} \tau$. However since the integrand is singular at $z=0$, a small neighbourhood around the origin, whose size is controlled by the parameter $\epsilon$, should be excluded from the integration domain. We found it convenient to take

$$
D_{\epsilon}=D /\left\{z:|z|<\frac{1}{2 \pi} \mathrm{e}^{-\gamma_{\mathrm{E}}} \epsilon\right\}
$$

where $\gamma_{\mathrm{E}}$ denotes the Euler constant. Then as $|\mathrm{q}| \rightarrow 0$

$$
Z_{\mathrm{EBH}}=\frac{1}{2 \pi} \sqrt{\frac{n}{\Im m(\tau)}}|\mathbf{q}|^{-\frac{1}{6}}\left(\log \left(4 \mathrm{e}^{\gamma_{\mathrm{E}}} / \epsilon\right)+o\left(|\mathbf{q}|^{0}\right)\right) .
$$

Through a numerical study, we found the following relation between the partition function of the Euclidean black hole NLSM and that which occurs in the scaling limit of the $\mathcal{Z}_{2}$ invariant spin chain:

$$
2 Z_{\mathrm{EBH}}=Z^{(\mathrm{cont})}+Z^{(\mathrm{disc})}
$$

Here $Z^{(\text {cont })}$ and $Z^{\text {(disc) }}$ are given by eqs. (5.33) and (7.18), respectively. Both sides of the above formula contain a divergent part $\propto \log (1 / \epsilon)$. For the Euclidean black hole NLSM this parameter regularizes the integral in (8.9), while for the lattice model $\epsilon \propto N^{-1}$ as in eq. (5.25). To perform a numerical check of (8.13) the divergent part needs to be subtracted. For this purpose, introduce the regularized partition function of the Euclidean black hole NLSM as

$$
Z_{\mathrm{EBH}}^{(\mathrm{reg})}=\lim _{\epsilon \rightarrow 0}\left(Z_{\mathrm{EBH}}-Z_{\epsilon}^{(\text {sing })}\right)
$$

with

$$
Z_{\epsilon}^{(\operatorname{sing})}=\sqrt{\frac{n}{\Im m(\tau)}} \frac{\log \left(4 \mathrm{e}^{\gamma_{\mathrm{E}}} / \epsilon\right)+\frac{1}{2} \log (\Im m(\tau))}{2 \pi(\overline{\mathrm{q}}, \overline{\mathrm{q}})_{\infty}^{2}(\mathrm{q}, \mathrm{q})_{\infty}^{2}} \sum_{\mathrm{u}, \mathrm{w} \in \mathbb{Z}} \overline{\mathrm{q}}^{-\frac{1}{12}+\frac{\bar{p}^{2}}{n+2}} \mathrm{q}^{-\frac{1}{12}+\frac{p^{2}}{n+2}}
$$

and recall that $\mathrm{q}=\mathrm{e}^{2 \pi \mathrm{i} \tau}, \overline{\mathrm{q}}=\mathrm{e}^{-2 \pi \mathrm{i} \tau^{*}}$. Here an extra term $\propto \log (\Im m(\tau))$ was included into the definition of $Z_{\epsilon}^{\text {(sing) }}$ in order to ensure that the regularized partition function is invariant under modular transformations in the case when $\mathrm{k}=0$ (for $\mathrm{k} \neq 0$ the partition function is not a modular invariant quantity). In turn, we define the regularized part of $Z^{\text {(cont) }}$ to be

$$
\begin{aligned}
Z_{\text {reg }}^{(\text {cont })=} & \sum_{\mathrm{u}, \mathrm{w} \in \mathbb{Z}} \int_{-\infty}^{+\infty} \mathrm{d} s \sum_{\mathrm{L}, \overline{\mathrm{L}} \geq 0} \tilde{\rho}_{\bar{p}, p}^{(\overline{\mathrm{L}}, \mathrm{L})}(s) \overline{\mathrm{q}}^{-\frac{1}{12}+\frac{s^{2}}{n}+\frac{\bar{p}^{2}}{n+2}+\overline{\mathrm{L}}} \mathrm{q}^{-\frac{1}{12}+\frac{s^{2}}{n}+\frac{p^{2}}{n+2}+\mathrm{L}} \\
& -\sqrt{\frac{n}{\Im m(\tau)}} \frac{\log \left(4 \mathrm{e}^{\gamma_{\mathrm{E}}}\right)+\frac{1}{2} \log (\Im m(\tau))}{\pi(\overline{\mathrm{q}}, \overline{\mathrm{q}})_{\infty}^{2}(\mathrm{q}, \mathrm{q})_{\infty}^{2}} \sum_{\mathrm{u}, \mathrm{w} \in \mathbb{Z}} \overline{\mathrm{q}}^{-\frac{1}{12}+\frac{\bar{p}^{2}}{n+2}} \mathrm{q}^{-\frac{1}{12}+\frac{p^{2}}{n+2}} .
\end{aligned}
$$

Here the density of states $\tilde{\rho}_{\bar{p}, p}^{(\overline{\mathrm{L}}, \mathrm{L})}(s)$ is given by eqs. $(5.28)-(5.30)$. Then (8.13) is equivalent to

$$
2 Z_{\mathrm{EBH}}^{(\mathrm{reg})}=Z_{\mathrm{reg}}^{(\mathrm{cont})}+Z^{(\mathrm{disc})} .
$$

The numerical data in support of this relation is presented in tables 1 and 2 . 


\begin{tabular}{|c|c|c|c|c|}
\hline$\tau$ & $Z_{\mathrm{reg}}^{(\mathrm{cont})}$ & $Z^{(\mathrm{disc})}$ & $Z_{\mathrm{reg}}^{(\text {cont }}+Z^{(\mathrm{disc})}$ & $2 Z_{\mathrm{EBH}}^{(\mathrm{reg})}$ \\
\hline$\tau=.9 \mathrm{i}$ & -3.9509313 & 0.0210525 & -3.9298787 & -3.9298786 \\
$-1 / \tau$ & -3.9358543 & 0.0059766 & -3.9298776 & -3.9298787 \\
$\tau+1$ & -3.9509313 & 0.0210525 & -3.9298787 & -3.9298786 \\
\hline$\tau=.2+.9 \mathrm{i}$ & -3.8983544 & 0.0065418 & -3.8918125 & -3.8918125 \\
$-1 / \tau$ & -3.8925978 & 0.0007853 & -3.8918125 & -3.8918124 \\
$\tau+1$ & -3.8983544 & 0.0065418 & -3.8918125 & -3.8918124 \\
\hline$\tau=.66 \mathrm{i}$ & -4.4682528 & 0.0943594 & -4.3738934 & -4.3738934 \\
$-1 / \tau$ & -4.3744476 & 0.0005542 & -4.3738934 & -4.3738933 \\
$\tau+1$ & -4.4682528 & 0.0943594 & -4.3738934 & -4.3738933 \\
\hline$\tau=.5 \mathrm{i}$ & -5.7668560 & 0.2960118 & -5.4708441 & -5.4708421 \\
$-1 / \tau$ & -5.4708761 & 0.0000322 & -5.4708439 & -5.4708437 \\
$\tau+1$ & -5.7668560 & 0.2960118 & -5.4708441 & -5.4708421 \\
\hline$\tau=.33 \mathrm{i}$ & -12.070612 & 1.5569389 & -10.513673 & -10.5129976 \\
$-1 / \tau$ & -10.513561 & $7.662 \cdot 10^{-8}$ & -10.513561 & -10.5135606 \\
$\tau+1$ & -12.070612 & 1.5569389 & -10.513673 & -10.5129975 \\
\hline
\end{tabular}

Table 1. A comparison of the numerical data for twice the regularized partition function of the Euclidean black hole NLSM (8.14) with $Z_{\text {reg }}^{\text {(cont) }}+Z^{\text {(disc) }}$ for the case $\mathrm{k}=0$ and $n=3$. Here $Z^{\text {(disc) }}$ is given by eqs. (7.17) and (7.18), while $Z_{\text {reg }}^{\text {(cont) }}$ is defined by (8.16). The table also illustrates modular invariance of the regularized partition function for $\mathrm{k}=0$. Note that in order to achieve good accuracy for decreasing values of $\Im m(\tau)$ one must take into account an increasing number of terms in the sum over $\mathrm{u}$ and $\mathrm{w}$ for $Z^{\text {(cont) }}$ as well as a and $\mathrm{b}$ in eq. (8.9). This significantly increases the computer time.

The following comments concerning some statements appearing in the literature are in order here. It was proposed in the works $[9,10]$ that $Z_{\mathrm{EBH}}(8.9)$ could be represented as

$$
\left.2 Z_{\mathrm{EBH}}\right|_{\mathrm{k}=0}=\sum_{\mathrm{u}, \mathrm{w}=-\infty}^{\infty} \int_{0}^{\infty} \mathrm{d} s 2 \rho(s) \operatorname{ch}_{\bar{p}, s}(\overline{\mathrm{q}}) \operatorname{ch}_{p, s}(\mathrm{q})+Z^{(\text {disc })} \quad(\text { from refs. }[9,10]) .
$$

The character entering into this formula is given by (3.44), while for the density of states

$$
\rho(s)=\frac{2}{\pi} \log (1 / \epsilon)+\frac{1}{2 \pi \mathrm{i}} \partial_{s} \log \left[\frac{\Gamma\left(\frac{1}{2}+p-\mathrm{i} s\right) \Gamma\left(\frac{1}{2}+\bar{p}-\mathrm{i} s\right)}{\Gamma\left(\frac{1}{2}+p+\mathrm{i} s\right) \Gamma\left(\frac{1}{2}+\bar{p}+\mathrm{i} s\right)}\right] .
$$

A numerical check of the consistency of (8.18) with (8.9) rules the conjecture out. Also the formula for the contribution of the discrete spectrum to the partition function $\left.Z_{\mathrm{EBH}}\right|_{\mathrm{k}=0}$ is 


\begin{tabular}{|c|c|c|c|c|}
\hline$\tau$ & $Z_{\text {reg }}^{(\text {cont }}$ & $Z^{(\mathrm{disc})}$ & $Z_{\mathrm{reg}}^{(\text {cont })}+Z^{(\mathrm{disc})}$ & $2 Z_{\mathrm{EBH}}^{(\mathrm{reg})}$ \\
\hline $0.9 \mathrm{i}$ & -3.1430392 & 0.0233941 & -3.1196452 & -3.1196450 \\
\hline $0.2+0.9 \mathrm{i}$ & -3.0646040 & 0.0099983 & -3.0546057 & -3.0546064 \\
\hline $0.66 \mathrm{i}$ & -3.7836669 & 0.1033699 & -3.6802970 & -3.6802972 \\
\hline $0.2+0.66 \mathrm{i}$ & -3.5074556 & 0.0418838 & -3.4655718 & -3.4655717 \\
\hline $0.50 \mathrm{i}$ & -5.1054421 & 0.3209649 & -4.7844771 & -4.7844724 \\
\hline $0.33 \mathrm{i}$ & -11.2855973 & 1.6391928 & -9.6464045 & -9.646289 \\
\hline $0.25 \mathrm{i}$ & -26.5761236 & 5.4010183 & -21.1751053 & -21.171536 \\
\hline
\end{tabular}

Table 2. The last column contains numerical data for $2 Z_{\mathrm{EBH}}^{(\mathrm{reg})}$ (8.14) with the parameters set to be $\mathrm{k}=-0.1$ and $n=3$. This is compared to $Z_{\mathrm{reg}}^{\text {(cont) }}+Z^{(\mathrm{disc})}$, where $Z^{\text {(disc) }}$ was computed using eqs. $(7.17),(7.18)$ and $Z_{\text {reg }}^{\text {(cont) }}$ via $(8.16)$.

given in ref. [11]. It appears to be identical with $\frac{1}{2} Z^{(\text {disc })}$ from (7.18) specialized to $\mathrm{k}=0$. However, eqs. (2.5) and (2.10) from ref. [11] do not quite correctly take into account the contribution of the states to $Z_{\mathrm{EBH}}^{(\mathrm{disc})}$ with $\mathfrak{j}=-\frac{n+1}{2},-\frac{1}{2}$ corresponding to the boundary of the interval in the set $\mathfrak{J}(\mathrm{v}, \mathrm{u})$ (7.17). Finally, the highly non-trivial formula (8.13) is in full agreement with the original observation of ref. [27]. However, let's emphasize that in order to state that the Euclidean black hole NLSM governs the critical behaviour of the $\mathcal{Z}_{2}$ invariant inhomogeneous six-vertex model, this relation is insufficient. Among others, the numerical study of the finite size corrections to the CFT Hamiltonian performed in [32], which are controlled by irrelevant perturbations, show that the extended conformal symmetry algebra is the $\bar{W}_{\infty} \otimes W_{\infty}$ - algebra with $c<2$.

In view of eqs. (8.17) and (8.16) one arrives at a conjecture for the equilibrium density matrix of the Euclidean black hole NLSM. Namely, being restricted to the level subspaces of the irreps of the $\bar{W}_{\infty} \otimes W_{\infty}$ - algebra belonging to $\mathcal{H}_{\mathrm{EBH}}^{\text {(cont) }}$, it is given by a formula similar to eq. (5.32):

$$
\left.\hat{\rho}_{\mathrm{EBH}}\right|_{\overline{\mathcal{W}}_{\bar{p}, s}^{(\mathrm{L})} \otimes \mathcal{W}_{p, s}^{(\mathrm{L})}}=\left[\frac{2}{\pi} \log (1 / \epsilon)+\frac{\tilde{\rho}_{\bar{p}, p}^{(\overline{\mathrm{L}}, \mathrm{L})}(s)}{\operatorname{par}_{2}(\overline{\mathrm{L}}) \operatorname{par}_{2}(\mathrm{~L})}\right] \overline{\mathrm{q}}^{-\frac{1}{12}+\frac{s^{2}}{n}+\frac{\bar{p}^{2}}{n+2}+\overline{\mathrm{L}}} \mathrm{q}^{-\frac{1}{12}+\frac{s^{2}}{n}+\frac{p^{2}}{n+2}+\mathrm{L}} \hat{\mathbf{1}}
$$

with $\tilde{\rho}_{\bar{p}, p}^{(\overline{\mathrm{L}} \mathrm{L})}(s)$ being defined via eqs. (5.28)-(5.30). It is important to keep in mind that the irreps appearing in the decomposition of $\mathcal{H}_{\mathrm{EBH}}^{\text {(cont) }}(8.5)$ are those of the $\bar{W}_{\infty} \otimes W_{\infty}$ algebra with $c=2+\frac{6}{n}>2$. The above formula is expected to be applicable to the case of twisted boundary conditions (8.1) with generic k. For the model with periodic boundary conditions, the density matrix is obtained via a taking of the limit $\mathrm{k} \rightarrow 0$. Some care 
is needed for the irreps with $p=\frac{1}{2} \mathrm{u}+\frac{1}{2}(n+2) \mathrm{k}, \bar{p}=\frac{1}{2} \mathrm{u}-\frac{1}{2}(n+2) \mathrm{k}$ and $\mathrm{u}$ odd, as $\tilde{\rho}_{\bar{p}, p}^{(\overline{\mathrm{L}} \mathrm{L})}(s)$ could contain simple poles at $s= \pm \frac{\mathrm{i}}{2}(n+2) \mathrm{k}$, which approach the real axis for vanishing $\mathrm{k}$. This gives rise to contact terms as in eq. (6.6). Finally $\hat{\rho}_{\mathrm{EBH}}$, being restricted to the discrete component $\mathcal{H}_{\mathrm{EBH}}^{(\text {disc })}$ of the space of states of the Euclidean black hole NLSM coincides with the usual thermal density matrix

$$
\left.\hat{\rho}_{\mathrm{EBH}}\right|_{\mathcal{H}_{\mathrm{EBH}}^{(\mathrm{disc})}}=\mathrm{q}^{L_{0}-\frac{c}{24}} \overline{\mathrm{q}}^{\bar{L}_{0}-\frac{c}{24}} .
$$

\section{Conclusion}

In this work we apply the results obtained for the $\mathcal{Z}_{2}$ invariant integrable spin chain to the study of two NLSMs. These are of interest since their target space geometries mimic that of a Lorentzian black hole and its Euclidean version.

The space of states occurring in the scaling limit of the low energy states of the spin chain contains both a discrete $\mathcal{H}^{\text {(disc) }}$ and continuous $\mathcal{H}^{\text {(cont) }}$ component. For the latter, the spectrum of conformal dimensions forms a continuous distribution which is characterized by a density of states. We conjecture that the pseudo-Hilbert space of the Lorentzian black hole NLSM coincides with a subspace of the $\mathcal{C}$ even sector of $\mathcal{H}^{\text {(cont) }}$ in the case of periodic boundary conditions for the spin chain. In turn, from the density of states restricted to this subspace we construct an equilibrium density matrix for the NLSM. An important point is that $\mathcal{H}^{(\text {disc })}$ is excluded from the identification. This was motivated through the study of the Hermitian structures for the spin chain, which suggests that the states from $\mathcal{H}^{(\mathrm{disc})}$ and $\mathcal{H}^{\text {(cont) }}$ can not be interpreted simultaneously as normalizable states within a single CFT.

Contrary to the Lorentzian black hole NLSM, the Hilbert space of the Euclidean one contains a discrete component made up of normalizable states, whose wavefunction(als) are localized in the vicinity of the tip of the target manifold. Remarkably, their contribution to the CFT partition function coincides with one half of the contribution of the states from $\mathcal{H}^{\text {(disc) }}$ to the partition function of the spin chain. Using the full density of states for $\mathcal{H}^{\text {(cont) }}$ an equilibrium density matrix for the Euclidean black hole NLSM is proposed, which reproduces the modular invariant partition function originally obtained by Maldacena, Ooguri and Son in ref. [9].

\section{Acknowledgments}

The authors thank H. Saleur, V. Schomerus and A. B. Zamolodchikov for stimulating discussions.

VB acknowledges the support of the Australian Research Council grant DP190103144.

The research of GK is funded by the Deutsche Forschungsgemeinschaft (DFG, German Research Foundation) under Germany's Excellence Strategy — EXC 2121 "Quantum Universe" - 390833306.

The work of SL is supported by the Rutgers New High Energy Theory Center. 
Open Access. This article is distributed under the terms of the Creative Commons Attribution License (CC-BY 4.0), which permits any use, distribution and reproduction in any medium, provided the original author(s) and source are credited.

\section{References}

[1] E. Witten, On string theory and black holes, Phys. Rev. D 44 (1991) 314 [InSPIRE].

[2] J.B. Hartle and S.W. Hawking, Path integral derivation of black hole radiance, Phys. Rev. D 13 (1976) 2188 [INSPIRE].

[3] S. Elitzur, A. Forge and E. Rabinovici, Some global aspects of string compactifications, Nucl. Phys. B 359 (1991) 581 [INSPIRE].

[4] G. Mandal, A.M. Sengupta and S.R. Wadia, Classical solutions of two-dimensional string theory, Mod. Phys. Lett. A 6 (1991) 1685 [InSPIRE].

[5] L.J. Dixon, M.E. Peskin and J.D. Lykken, $N=2$ superconformal symmetry and $\mathrm{SO}(2,1)$ current algebra, Nucl. Phys. B 325 (1989) 329 [INSPIRE].

[6] R. Dijkgraaf, H.L. Verlinde and E.P. Verlinde, String propagation in a black hole geometry, Nucl. Phys. B 371 (1992) 269 [INSPIRE].

[7] A.B. Zamolodchikov and Al.B. Zamolodchikov, unpublished notes, (1995).

[8] J.M. Maldacena and H. Ooguri, Strings in $A d S_{3}$ and $\mathrm{SL}(2, R)$ WZW model. I: the spectrum, J. Math. Phys. 42 (2001) 2929 [hep-th/0001053] [INSPIRE].

[9] J.M. Maldacena, H. Ooguri and J. Son, Strings in $A d S_{3}$ and the $\mathrm{SL}(2, R)$ WZW model. II: Euclidean black hole, J. Math. Phys. 42 (2001) 2961 [hep-th/0005183] [INSPIRE].

[10] A. Hanany, N. Prezas and J. Troost, The partition function of the two-dimensional black hole conformal field theory, JHEP 04 (2002) 014 [hep-th/0202129] [INSPIRE].

[11] S. Ribault and V. Schomerus, Branes in the 2D black hole, JHEP 02 (2004) 019 [hep-th/0310024] [INSPIRE].

[12] V. Schomerus, Non-compact string backgrounds and non-rational CFT, Phys. Rept. 431 (2006) 39 [hep-th/0509155] [INSPIRE].

[13] E. Witten, Non-Abelian bosonization in two-dimensions, Commun. Math. Phys. 92 (1984) 455 [INSPIRE].

[14] K. Gawȩdzki and A. Kupiainen, Coset construction from functional integrals, Nucl. Phys. B 320 (1989) 625 [INSPIRE].

[15] L.D. Faddeev and L.A. Takhtajan, Hamiltonian methods in the theory of solitons, Springer, Berlin, Heidelberg, Germany (1987).

[16] V.A. Fateev and S.L. Lukyanov, Poisson Lie groups and classical W algebras, Int. J. Mod. Phys. A 7 (1992) 853 [INSPIRE].

[17] I. Bakas and E. Kiritsis, Beyond the large $N$ limit: nonlinear $W(\infty)$ as symmetry of the $\mathrm{SL}(2, R) / \mathrm{U}(1)$ coset model, Int. J. Mod. Phys. A 7 (1992) 55 [hep-th/9109029] [InSPIRE].

[18] V.G. Knizhnik and A.B. Zamolodchikov, Current algebra and Wess-Zumino model in two-dimensions, Nucl. Phys. B 247 (1984) 83 [INSPIRE].

[19] A.B. Zamolodchikov, Montreal talk, unpublished, (1985). 
[20] M. Wakimoto, Fock representations of the affine lie algebra $A_{1}^{(1)}$, Commun. Math. Phys. 104 (1986) 605 [INSPIRE].

[21] A. Gerasimov, A. Marshakov and A. Morozov, Free field representation of parafermions and related coset models, Nucl. Phys. B 328 (1989) 664 [Theor. Math. Phys. 83 (1990) 466] [Teor. Mat. Fiz. 83 (1990) 186] [InSPIRE].

[22] T. Jayaraman, K.S. Narain and M.H. Sarmadi, $\mathrm{SU}(2)_{k} W Z W$ and $Z_{k}$ parafermion models on the torus, Nucl. Phys. B 343 (1990) 418 [InSPIRE].

[23] R.J. Baxter, Generalized ferroelectric model on a square lattice, Stud. Appl. Math. 50 (1971) 51 [INSPIRE].

[24] J.L. Jacobsen and H. Saleur, The antiferromagnetic transition for the square-lattice Potts model, Nucl. Phys. B $\mathbf{7 4 3}$ (2006) 207 [cond-mat/0512058] [INSPIRE].

[25] Y. Ikhlef, J. Jacobsen and H. Saleur, A staggered six-vertex model with non-compact continuum limit, Nucl. Phys. B 789 (2008) 483 [cond-mat/0612037].

[26] Y. Ikhlef, J.L. Jacobsen and H. Saleur, The $\mathbb{Z}_{2}$ staggered vertex model and its applications, J. Phys. A 43 (2010) 225201 [arXiv:0911.3003].

[27] Y. Ikhlef, J.L. Jacobsen and H. Saleur, An integrable spin chain for the $\mathrm{SL}(2, R) / \mathrm{U}(1)$ black hole sigma model, Phys. Rev. Lett. 108 (2012) 081601 [arXiv:1109.1119] [INSPIRE].

[28] H. Frahm and M.J. Martins, Phase diagram of an integrable alternating $U_{q}[s l(2 \mid 1)]$ superspin chain, Nucl. Phys. B 862 (2012) 504 [arXiv:1202.4676] [INSPIRE].

[29] C. Candu and Y. Ikhlef, Nonlinear integral equations for the $\mathrm{SL}(2, R) / \mathrm{U}(1)$ black hole sigma model, J. Phys. A 46 (2013) 415401 [arXiv:1306.2646] [inSPIRE].

[30] H. Frahm and A. Seel, The staggered six-vertex model: conformal invariance and corrections to scaling, Nucl. Phys. B $\mathbf{8 7 9}$ (2014) 382 [arXiv:1311.6911] [INSPIRE].

[31] V.V. Bazhanov, G.A. Kotousov, S.M. Koval and S.L. Lukyanov, On the scaling behaviour of the alternating spin chain, JHEP 08 (2019) 087 [arXiv: 1903.05033] [INSPIRE].

[32] V.V. Bazhanov, G.A. Kotousov, S.M. Koval and S.L. Lukyanov, Scaling limit of the $\mathcal{Z}_{2}$ invariant inhomogeneous six-vertex model, Nucl. Phys. B 965 (2021) 115337 [arXiv: 2010.10613] [INSPIRE].

[33] J.L. Cardy, Operator content of two-dimensional conformally invariant theories, Nucl. Phys. B 270 (1986) 186 [INSPIRE].

[34] G.A. Kotousov and S.L. Lukyanov, Spectrum of the reflection operators in different integrable structures, JHEP 02 (2020) 029 [arXiv: 1910.05947] [INSPIRE].

[35] D. Gepner and Z.-A. Qiu, Modular invariant partition functions for parafermionic field theories, Nucl. Phys. B 285 (1987) 423 [InSPIRE].

[36] P.A. Griffin and O.F. Hernandez, Feigin-Fuchs derivation of $\mathrm{SU}(1,1)$ parafermion characters, Nucl. Phys. B 356 (1991) 287 [INSPIRE]. 